PALEO

Revue d'archéologie préhistorique

$20 \mid 2008$

Spécial table ronde ( $2^{\mathrm{e}}$ partie) : Le Gravettien : entités régionales d'une paléoculture européenne, Les Eyzies, juillet 2004

\title{
Comportement des populations d'Europe au Gravettien : pratiques funéraires et interprétations
}

Behaviours of Gravettian European populations : funerary practices and interpretations

Dominique Henry-Gambier

\section{OpenEdition}

Édition électronique

URL : http://journals.openedition.org/paleo/1632

DOI : $10.4000 /$ paleo. 1632

ISSN : 2101-0420

Éditeur

SAMRA

Édition imprimée

Date de publication : 1 décembre 2008

Pagination : 399-438

ISSN : 1145-3370

\section{Référence électronique}

Dominique Henry-Gambier, «Comportement des populations d'Europe au Gravettien : pratiques funéraires et interprétations », PALEO [En ligne], 20 | 2008, mis en ligne le 13 mai 2011, consulté le 07 juillet 2020. URL : http://journals.openedition.org/paleo/1632 ; DOI : https://doi.org/10.4000/paleo. 1632

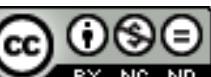

PALEO est mis à disposition selon les termes de la licence Creative Commons Attribution - Pas d'Utilisation Commerciale - Pas de Modification 4.0 International. 


\title{
COMPORTEMENT DES POPULATIONS D'EUROPE AU GRAVETTIEN : PRATIQUES FUNÉRAIRES ET INTERPRÉTATIONS
}

\author{
Dominique HENRY-GAMBIER ${ }^{(1)}$
}

\begin{abstract}
Résumé : Cet article est une révision des assemblages de vestiges humains gravettiens classiquement interprétés comme des dépôts funéraires. Les documents anthropologiques gravettiens sont nombreux mais tous ne proviennent pas de fouilles exemplaires et les données brutes sont souvent vagues, voire contradictoires. II est évidemment impossible de reconstituer a posteriori, les informations qui auraient du être enregistrées lors des fouilles. Toutefois, une réflexion conduite à partir des vestiges humains tels qu'ils existent aujourd'hui, des archives lorsqu'elles sont accessibles, des acquis de l'Anthropologie de terrain et des nouvelles méthodes de détermination de l'âge au décès et du sexe, permet de fonder plus sérieusement une restitution des gestes funéraires. Cette restitution constitue une étape préalable à la discussion sur la reconnaissance d'entités culturelles régionales et sur leurs éventuelles relations ainsi que sur l'évolution et la signification des comportements funéraires. La première partie est consacrée à la présentation de l'échantillon et à la discussion du caractère intentionnel des dépôts et aux données biologiques (recrutement, âge, sexe et pathologie). La seconde partie concerne les caractéristiques de ces dépôts (sépultures primaire/secondaires, dispositif funéraire, mobilier et parure). La troisième partie est une tentative de synthèse et d'interprétation.
\end{abstract}

Mots-clés : Paléolithique supérieur, Gravettien, Homo sapiens sapiens, pratiques funéraires, Europe.

Abstract: Behaviours of Gravettian European populations: funerary practices and interpretations. This article is a review of Gravettian human remains interpreted as funerary deposits. In Europe, the gravettian human remains are numerous. As many excavations took place at the end of the $19^{\text {th }}$ century, the reliability of the data is sometime questionable. It is impossible to recreate missing information. However, reflection on the human bones using records, publications, the method of the "field anthropology" and recent method to determine sex and individual age at death allow to reconstruct more securely funerary practices. This work is a preliminary phase to recognize cultural and regional groups, to discuss relations between these groups and evolution of the funerary behaviours. First we will examine the European sample, the arguments in favour intentional deposits and the biological data (sex, age at death, pathology, death cause). The second part concerns the characteristics of the burials (primary/secondary burial- architecture of the tomb, grave goods,....). In the third part, we propose a synthesis and we discuss interpretations.

Key-words: Upper Palaeolithic, Gravettian, Homo sapiens sapiens, funerary practices, Europe.

\section{INTRODUCTION}

Les rites funéraires font partie de l'identité culturelle d'un groupe. Les données historiques et ethnographiques montrent que les sociétés donnent au problème de la mort des réponses très diverses. À travers les gestes et les rites mis en œuvre autour du décès d'un individu, la société s'exprime : âge du défunt, genre, statut, rôle, cause et contexte de la mort sont autant de paramètres susceptibles de déterminer les traitements et les rituels (Thomas 1980).
Aussi, les tombes et plus généralement les pratiques funéraires représentent une source potentielle d'informations sur l'organisation et le fonctionnement des sociétés, les comportements des hommes ainsi que sur leurs mentalités et leurs croyances.

Dans le domaine archéologique, les données sur les comportements funéraires se réduisent aux seuls témoignages matériels : ossements du défunt, dispositif funéraire, mobilier. La restitution de ces comportements est donc forcé-

(1) Université Bordeaux 1, UMR 5199, PACEA - Laboratoire d'Anthropologie des Populations du Passé, avenue des Facultés, 33405 Talence, France - d.gambier@anthropologie.u-bordeaux1.fr 
ment limitée et l'on doit s'interroger sur notre capacité avec de tels documents à approcher le sens et les croyances qui sous-tendent chaque geste mis en évidence. Un même geste ou plutôt sa trace, peut avoir plusieurs significations. De la même façon, si les relations entre organisation sociale et pratiques funéraires sont effectives, la variation des coutumes funéraires dans les sociétés traditionnelles selon les dimensions, l'organisation sociale et économique du groupe est complexe aussi les liens entre gestes funéraires et société ne peuvent être directs.

Pour le Paléolithique, la rareté des vestiges, l'hétérogénéité de la documentation (qualité inégale des fouilles et conservation différentielle, ...) constituent des handicaps supplémentaires. Les tentatives de synthèse et les études de cas les plus récentes témoignent de ces difficultés (Binant 1991; Buzhilova 2000 ; Formicola 1988, 1991; Formicola et al. 200 ; 2002; Gambier 1996, 2000 ; Henry-Gambier 2001, 2005 ; Jelinek 1991, 1992 ; May 1986 ; Mussi 1986a ; Mussi et al. 1989 ; Oliva 2000, 2000-2001 ; Orschiedt 2002 ; Riek Salvatore et al. 2001 ; Svoboda 1997 ; Svoboda 2008 a et b ; Ullrich 1997 ; Vanharen et al. 2001 ; Vanharen 2002 ; Vanharen et al. 2003 a, 2003 b ; Zilhao et al. 2002 ; Zilhao 2005). Dans la plupart de ces travaux, le discours s'appuie encore trop peu sur les données anthropologiques. Les méthodes de l'Anthropologie de terrain ${ }^{2}$ développées en France à partir de l'analyse des ensembles funéraires des populations plus récentes (Duday 2005 ; Duday et al. 1990) sont ignorées. Les changements de paradigme dans la diagnose de l'âge au décès et du sexe ne sont guère pris en considération (Murail et al. 2000, 2005 ; Schmitt 2001, 2002 ...). Les faits archéologiques et anthropologiques sollicités à l'appui de la restitution des gestes funéraires ne sont pas toujours pertinents et vérifiés ou sont sur-interprétés, donnant une image illusoire des pratiques funéraires des hommes du Paléolithique et des systèmes de pensées qui les sous-tendent.

Cet article est une révision des assemblages de vestiges humains gravettiens classiquement interprétés comme des dépôts funéraires. Les documents anthropologiques gravettiens sont nombreux mais tous ne proviennent pas de fouilles exemplaires et les données brutes sont souvent vagues, voire contradictoires. II est évidemment impossible de reconstituer a posteriori, les informations qui auraient du être enregistrées lors des fouilles. Toutefois, une réflexion conduite à partir des vestiges humains tels qu'ils existent aujourd'hui, des archives lorsqu'elles sont accessibles et des acquis de l'Anthropologie de terrain, permet de fonder plus sérieusement une restitution des gestes funéraires, étape préalable à la discussion sur la reconnaissance d'entités culturelles régionales et sur leurs éventuelles relations ainsi que sur les comportements, leur évolution et leur signification.

La première partie sera consacrée à la présentation de l'échantillon et à discussion du caractère intentionnel des dépôts et aux données biologiques (recrutement, âge, sexe et pathologie). La seconde partie concernera les caractéristiques de ces dépôts (sépultures primaire/secondaires, dispositif funéraire, mobilier et parure). La troisième partie est une tentative de synthèse et d'interprétation.

\section{1 - PRÉSENTATION DE L'ÉCHANTILLON ET DONNÉES BIOLOGIQUES}

\section{La documentation anthropologique}

En Europe, 28 sites gravettiens ou contemporains (tab. 1 - en annexe) auraient livré des vestiges humains relevant d'un possible traitement funéraire ${ }^{3}$. Les datations radiocarbone directes en SMA des fossiles ou d'objet associés, la révision des industries et des sites ont permis dans la dernière décennie de réévaluer la position chronologique et culturelle de découvertes anciennes (tab. 2, 3, 4 - en annexe). De nouvelles découvertes en République Tchèque, France, Italie, Portugal et Autriche ont enrichi l'échantillon. Des incertitudes persistent cependant. Ainsi, Combe-Capelle (Dordogne) retrouvé récemment à Berlin (Hoffmann et al. 2002), a été exhumé par $O$. Hauser au début du siècle avec des méthodes trop discutables pour accepter une attribution au Gravettien sans datation $\mathrm{C}^{14}$ des vestiges humains. Et ce d'autant plus qu'existent des indices de sépultures récentes dans ce gisement (Gambier et al. 2000). Les vestiges de Brno -Zabvresky, Brno Cerveny Kobe en Moravie, trouvés sans contexte culturel (Oakley et al. 1971) ne sont pas rapportables au Gravettien sans faire des hypothèses invérifiables. Plusieurs des spécimens trouvés au XIXè siècle dans les grottes des BaousséRoussé en Ligurie n'ont toujours pas de position culturelle définitive, même si l'appartenance au Gravettien est la plus vraisemblable (Mussi 1986 b; Henry-Gambier 2001, 2005). L'attribution au Gravettien (Onoratini et al. 1996) de l'enfant du Figuier (Ardèche) est aussi à vérifier par une datation ${ }^{14} \mathrm{C}$ car la grotte a été occupée du Gravettien au Néolithique. De même devrait être clarifiée la position chronologique et culturelle des fossiles de Kostenki (Russie) attribués à des unités culturelles distinctes ou proches du Gravettien (Sinitsyn 2004). Les datations radiocarbones sont en effet, très dispersées (tab. 4 - en annexe).

(2) Approche dont l'objectif est de restituer les gestes funéraires. Elle place les vestiges humains au centre de l'analyse et pour cette raison exige une très bonne connaissance de l'os humain et ce dès le terrain, lieu privilégié d'observation et d'enregistrement. Elle accorde aussi une place majeure aux processus taphonomiques ayant affecté le dépôt initial, processus qui parce qu'ils sont aussi liés à la structure de la tombe, renseignent sur les pratiques funéraires (Duday, 2005).

(3) Cet inventaire n'est pas exhaustif. D'autres sites gravettiens ont livré des vestiges humains mais leur condition de découverte ou leur état de conservation nous ont conduit à les exclure de cet inventaire. De même, la découverte dans le gisement de Vilhonneur (Charente) d'un squelette humain et d'au moins cinq squelettes de hyènes dans une cavité profonde dont les parois portaient des peintures pariétales (Henry-Gambier et al. 2007) n'est pas intégrée dans ce travail, les fouilles n'ayant pas débuté. 
L'échantillon étudié ici, comprend donc des fossiles situés dans un intervalle de temps d'environ 6 -7 millénaires (28$21000 \mathrm{BP})$. II provient d'une aire géographique vaste. Certaines régions pourtant peuplées au Gravettien n'ont livré aucun document anthropologique. L'Europe centrale et orientale ainsi que l'Italie sont plus riches que l'extrême ouest de l'Europe (France, Péninsule ibérique et Grande-Bretagne). Cette répartition correspond à des contextes géographiques et environnementaux variés, à des techno-complexes particuliers même s'ils s'inscrivent dans une même tradition (Djinjian et al. 1999 ; Otte et al. 2004 ; Svoboda 2003...).

\section{Dépôt intentionnel}

\section{Remarques méthodologiques}

Avant de discuter d'un assemblage d'ossements humains en terme de pratique funéraire, il est nécessaire de prouver le caractère intentionnel du dépôt. Les débats autour de l'existence des sépultures néanderthaliennes (Tillier 1990) démontrent combien cette question essentielle pour toutes les périodes (Leclerc 1990), est d'autant plus complexe pour le Paléolithique que les données sont souvent partielles ou de fiabilité faible, les observations sur le terrain ayant été trop hâtives.

Dans le registre archéologique, les critères classiquement utilisés pour affirmer l'intentionnalité d'un dépôt sont les suivants : 1) présence de fosse anthropique (1A) et/ou d'éléments architecturaux (1B) tels que pierres, ossements, structure en bois ; 2) association d'objets mobiliers (la parure ne peut être considérée car elle est liée à l'individu et peut l'accompagner lors d'un décès accidentel) ;

3) présence de colorant ;

4) taux de représentation du ou des squelettes ;

5) connexions anatomiques ;

6) position du ou des corps ;

7) nombre d'individus ;

8) localisation des vestiges dans un lieu fréquenté par les hommes (habitat en grotte, abri ou plein air, grotte « sanctuaire »).

Ces critères n'ont pas la même valeur. Pris séparément, les critères 1, 2, 3 signent sans équivoque un dépôt intentionnel. Dans le cas du colorant, il faut cependant s'assurer qu'il ne relève pas d'actions techniques sans lien avec les ossements humains. Son extension et ses relations avec les os humains doivent être précisées. En revanche, le taux de représentation du squelette (Cr.4), la préservation des connexions anatomiques (Cr.5) ne sont pas des critères univoques de dépôt intentionnel. En effet, un décès accidentel suivi d'un enfouissement naturel et immédiat peut permettre une bonne conservation du squelette et la préservation des connexions. En revanche, le critère 6 est important : une position des corps telle que décubitus latéral ou dorsal avec flexion ou hyper flexion des membres a en effet, peu de chance d'être due au hasard. Elle implique une manipulation du corps et par conséquent une intervention humaine. De la même façon, le nombre d'individus (Cr.7) représente un indice fort de dépôt volontaire. L'enfouissement naturel et rapide de plusieurs sujets décédés accidentellement est plausible, mais l'enchaînement d'événements qu'exige ce cas de figure paraît improbable. Le critère 8 n'est pas univoque, toutefois la réunion dans un même espace des artisans et de leur production rend la thèse de l'accident et de l'abandon peu crédible.

Si pris isolément, les critères $4,5,7,8$ ne sont pas univoques d'une action intentionnelle, leur combinaison peut permettre de poser un diagnostic de dépôt intentionnel. Par exemple, l'association des critères 4, 7, 8 (plusieurs individus contemporains représentés par une part significative du squelette, dans un lieu occupé par les hommes) constitue en l'absence de toute cause naturelle expliquant cet assemblage, une forte présomption de dépôt intentionnel.

II est important d'insister sur le fait que le désordre d'un assemblage osseux, le déficit en certains os et/ou l'absence d'éléments architecturaux, ne permettent pas de réfuter l'intentionnalité du dépôt. D'éventuelles causes naturelles ou accidentelles peuvent expliquer l'assemblage osseux observé. Aussi, le raisonnement doit intégrer l'analyse des processus taphonomiques. Cette approche implique une connaissance de la dynamique sédimentaire (mise en place et évolution des dépôts, érosion, ruissellement, dépôts de concrétions, solifluxion, ....) et la recherche sur les os des traces (rongements par les carnivores, stigmates de charriage, dégradations physico-chimiques, altérations anthropiques...). La démonstration est délicate voire impossible lorsque l'assemblage osseux initial a été trop perturbé (cas des vestiges humains épars trouvés en situation apparemment détritique dans des niveaux d'habitat) ou lorsque l'ancienneté de la découverte nous prive des données pertinentes.

\section{Échantillon gravettien}

Dans l'échantillon gravettien, 45 individus (non compris les sujets de Predmost) représentent des dépôts intentionnels répondant à au moins un des trois premiers critères (tabl. 5, 6 et 7 en annexe). Toutefois, la dent isolée et le fragment de crâne d'enfant des Marronniers (Onoratini et al. 1996) trouvés dans une fosse avec de l'ocre et de la parure relèvent plus certainement d'une association fortuite car d'autres vestiges humains aussi fragmentaires, des parures et de l'ocre étaient dispersés dans le même niveau.

Les ensembles de Kostenki 8 et 12, Barma Grande 6, Baousso da Torre 3, Cussac ${ }^{4}$, Pataud, Paglicci (A, B, C, D, F, G, H, I, J, L, M, N), Pavlov I ${ }^{5}$ (2- 28), Dolni Vestonice I (12; 4- 10; 23-32; 35; 37-38), Dolni Vestonice II (11, 12, 17, 33,

(4) Les recherches sur le site de Cussac n'ont pas débuté pour diverses raisons (le statut juridique du site, la préservation et le taux élevé de CO2 qui limite la fréquentation du site). Les faits présentés dans ce travail résultent de d'observations faites in situ et à distance lors des premières expertises.

(5) Les gisements de la colline de Pavlov en R. Tchèque : Pavlov, Dolni Vestonice comprennent plusieurs secteurs définis par un chiffre romain (Pavlov I, Dolni Vestonice I et II). Les chiffres arabes désignent les individus (Pavlov 1, Dolni Vestonice 14, 15, 16). 
34, 36, 39, 40 à 53), Soungir 4-9, La Rochette 1-2, ne satisfont pas aux critères 1,2,3 (tabl. 5,6 et 7 en annexe) et doivent être discutés. L'ensemble de Predmost révisé récemment (Svoboda 2008 a et b) mérite d'être traité à part.

La position du corps (Cr.6) et le taux de représentation du squelette (Cr.4) de Kostenki 12 (Sinitsyn 2004) sont en faveur d'un dépôt intentionnel. Le regroupement des os brûlés (134 fragments crâniens, des phalanges, des fragments de vertèbres et des dents) de Kostenski 8 dans un habitat près d'un foyer (Sinitsyn 2004) évoque un geste intentionnel mais une étude détaillée de cet ensemble serait nécessaire, le contact fortuit entre des os et le feu étant fréquent dans le contexte d'un habitat. L'originalité de la position (sur le ventre) de Baousso da Torre 3 pourrait évoquer un « abandon ». Toutefois, sa localisation dans un lieu (Cr.8) et dans un niveau ayant livré deux dépôts intentionnels (BT1 et BT2) très proches plaide en faveur d'un dépôt intentionnel (Henry-Gambier 2001, 2005). Les mêmes critères conduisent à inclure dans l'ensemble des dépôts intentionnels Barma Grande 6. Le taux de représentation des squelettes de Cussac, le nombre d'individus (Cr.7), leur répartition le long d'un circuit intégrant des panneaux gravés contemporains ( $\mathrm{Cr}$. 8) et leur gisement dans des conditions similaires (dans des bauges d'ours non colmatées), suggèrent un acte volontaire (Aujoulat et al. 2002). À Pataud, la localisation dans un même niveau d'habitat (Cr. 8) et dans un espace réduit (le fond de l'abri), le taux de représentation des squelettes ( $\mathrm{Cr}$. 4), le nombre d'individus (Cr. 7) ainsi que la préservation d'une partie des connexions anatomiques d'un des sujets sont aussi en faveur d'un acte intentionnel (Nespoulet et al. sous-presse).

Les vestiges isolés et fragmentaires des sites de Paglicci, Pavlov I, Dolni Vestonice I et II ne répondent qu'au critère 8 (os trouvés dans un lieu très anthropisé ayant livré des dépôts intentionnels). Les données sur leur dispersion et sur leur état de surface (Borgognini et al. 1980; Sladek et al. 2000) ne permettent pas d'argumenter en faveur d'un geste intentionnel. Soungir 4 correspond à un fragment de fémur d'adulte cassé anciennement et trouvé près de l'épaule gauche de Soungir 2 (Buzhilova et al. 2000) : Elément du mobilier déposé volontairement ou os «arrivé» fortuitement lors du colmatage de la fosse ? II est impossible de conclure. L'ocre sur Soungir 5 , une calotte crânienne découverte au sommet du remplissage de la fosse de Soungir 1, n'est pas ici un indice suffisant pour conclure à un dépôt volontaire, l'ocre étant abondant dans tout le niveau. Quant aux assemblages osseux constitués par Soungir 6, 7, 8, 9 (fragments de squelettes), ils sont inédits et les os sont perdus (Buzhilova et al. 2000)! Enfin, les informations sur la Rochette 1-2 sont trop pauvres pour statuer sur la nature du dépôt.

L'ensemble de Predmost est constitué d'une trentaine (?) d'individus découverts avant 1930 dans le secteur PI du site. Quelques os étaient dispersés mais la majorité d'entre eux étaient regroupés sur une surface d'environ $10 \mathrm{~m} 2$ (fouilles Maska 1894). Ici, la concentration à proximité immédiate d'un habitat riche (Svoboda 2008 a et b) et le nombre d'individus plaident en faveur de l'intentionnalité.
A l'issue de cette révision, les dépôts de vestiges fragmentaires du Marronnier, de Paglicci, de Dolni Vestonice I, II et Pavlov I, Soungir $(4,5,6,7,8,9)$ et La Rochette 1, 2 ne peuvent donc être retenus comme des dépôts intentionnels.

\section{Dénombrement des individus}

\section{Remarques méthodologiques}

Le dénombrement des individus est complexe dans les dépôts qui associent plusieurs individus et qui ont été perturbés anciennement ou au moment de la fouille (absence de connexions anatomiques ou connexions non relevées). La reconnaissance des os d'un même sujet repose sur l'étude anthropologique avec la recherche des liaisons de deuxième ordre : collage de fragments jointifs, contiguïté articulaire, identité de stade de maturation, appartenance à un même ensemble pathologique et appariement d'os symétriques (Duday 2005).

\section{Échantillon gravettien}

La majorité des dépôts sont individuels. La question du dénombrement est particulièrement problématique pour le site de Predmost. La destruction des os pendant la seconde guerre mondiale empêche toute réévaluation exacte du nombre initial d'individus. II varie de 15 à 30 . Le chiffre de quatre adultes à Cro-Magnon repose sur le décompte des os longs mais le regroupement par individu est problématique car la recherche des liaisons de second ordre se heurte à la fragmentation, à l'érosion (ancienne et récente) des os et/ou aux produits de restauration qui masquent souvent les cassures. Un problème analogue existe pour les ensembles de l'Abri Pataud (Henry-Gambier et al. 2005 ; Villotte 2008).

Enfin le dénombrement des individus à Cussac est fondé sur le nombre d'humérus identifiés à partir d'observations réalisées in situ et à distance. L'étude ostéologique directe modifiera sans doute le résultat actuel $(\mathrm{NMI}=5)$.

Au total, c'est une soixantaine d'individus qui ont fait l'objet d'un dépôt intentionnel.

\section{Sexe et âge au décès}

\section{Remarques méthodologiques}

Une détermination fiable de l'âge au décès et du sexe est un préalable à la caractérisation et à la compréhension des comportements funéraires. Les recherches des dix dernières années sur le dimorphisme sexuel des adultes prouvent que les méthodes de diagnose basées sur la morphologie ou la robustesse du crâne et du squelette ont une fiabilité très basse. De même, la synostose des sutures crâniennes ou l'usure de l'émail des dents se situent parmi les plus mauvais indicateurs d'âge au décès des adultes (Ball 2002 ; Key et al. 1994 ; Schmitt 2001). Ces méthodes appliquées aux populations paléolithiques tendent à surestimer le nombre d'hommes (les femmes robustes sont classées parmi les hommes) et à sous-estimer le nombre de sujets âgés. 
Ces dernières années, la mise au point de méthodes reposant sur un vaste échantillon de sexe et d'âge connus représentatif de la variabilité mondiale et sur un principe de décision statistique (Diagnose probabiliste du sexe- DSP) a ouvert de nouvelles perspectives. La diagnose est fondée sur une approche morphologique et métrique de l'os coxal. Cette approche défend l'idée d'un dimorphisme commun à Homo sapiens sapiens et s'appuie sur la notion de compensation du dimorphisme sexuel entre les segments morpho fonctionnels de l'os coxal impliquant plusieurs variables qualitatives et quantitatives (Bružek 2002 ; Murail et al. 2005). Testées sur des échantillons de populations actuelles d'âge et de sexe connus, ces méthodes ont une fiabilité très élevée. En outre, elles permettent une diagnose fiable pour des sujets dont l'os coxal est fragmentaire. En l'absence de l'os coxal, la détermination du sexe à partir de la diagnose probabiliste secondaire (Murail et al. 1999) apparaît prometteuse (Villotte et al. 2007 ; Villotte 2008). Elle utilise comme référence l'échantillon d'individus du Paléolithique supérieur " sexés " selon la $D S P$ et/ou l'approche morphoscopique (Bružek 2002).

Pour l'âge individuel au décès des adultes, les difficultés de détermination tiennent à la grande variabilité des modifications des indicateurs osseux et à leur faible corrélation avec l'âge. La méthode basée sur l'évolution de la région sacro pelvienne au cours de la sénescence proposée par Schmitt (2001) contourne cette difficulté à partir d'une approche probabiliste bayésienne. L'âge est estimé sous forme d'intervalles chronologiques larges, favorisant ainsi la fiabilité de l'estimation plutôt que la précision.

Pour les immatures, l'estimation de l'âge au décès repose sur le développement de la denture et sur l'ossification du squelette en référence à des standards actuels (Moorrees et al. 1963 ; Scheuer et al. 2000 ; Ubelaker 1978). II s'agit d'un âge biologique et l'écart entre âge chronologique et biologique est inconnu.

En revanche, la diagnose du sexe des immatures est un problème non résolu en dépit des recherches engagées (Majo 1992 ; Majo et al. 1993) car l'ampleur du dimorphisme sexuel avant la puberté est trop faible pour permettre une diagnose fiable. Une détermination à partir de l'ADN fossile est possible mais le caractère destructeur de cette méthode, le faible pourcentage de résultats, les problèmes de pollution par de l'ADN récent et le coût la rendent peu opérationnelle pour l'instant.

\section{Échantillon gravettien}

Les résultats obtenus lors de l'application de ces méthodes sont présentés dans les tableaux 8 et 9 .

Le nombre d'adultes ayant fait l'objet d'une sépulture est de 36 (Predmost non compris). 9 femmes et 16 hommes (le sexe de quatre d'entre eux mériterait d'être vérifié par une méthode fiable) ont été identifiés. Onze sont de sexe incon- nu (sujet inédits tels que ceux de Cussac, sujets insuffisamment conservés ou sujet dont le dimorphisme sexuel est faible). Le jeune adulte, Dolni Vestonice 15, au centre de la sépulture triple, est classé parmi les hommes à partir de la morphologie de l'os coxal. Formicola et al. (2001) l'attribuent à une femme car les pathologies qui l'affectent, seraient léthales chez l'homme mais l'étiologie de ces pathologies ne fait pas consensus (Klima 1987 b ; Jelinek 1992 ; Kuklik 1992 ; Formicola et al. 2001 ; Trinkaus et al. 2001 ; Trinkaus et al. 2006). Parmi les adultes (tabl. 9 en annexe) de Predmost, on compte quatre hommes et une femme "sexés" par une méthode fiable (Bružek et al. 2008).

Les 22 immatures (Predmost non inclu), comprennent des individus âgés de moins de cinq ans dont plusieurs immatures au stade périnatal (Pataud, Cro-Magnon, Kostenki, Krems) ainsi que des sujets se situant entre huit-neuf ans et la fin de l'adolescence. Leur sexe n'est pas déterminable à partir du squelette pour les raisons évoquées cidessus, excepté Dolni Vestonice 14 à la limite adolescence/âge adulte (tabl. 8 et 9 - en annexe).

La détermination du sexe des enfants de Soungir ${ }^{6}$ à partir de l'ADN (Poltoraus et al. 2000) n'est pas recevable. L'analyse n'a pas été répliquée dans un second laboratoire et l'authenticité de l'ADN analysé est contestée (Ovchinnikov et al. 2003).

A Predmost, des immatures dont trois au stade périnatal auraient été déposés dans le même espace que les adultes (Bružek et al. 2008). L'un des sujets, Predmost 9, à la limite adolescence/âge adulte serait une femme (tabl. 9 en annexe).

\section{Pathologies et causes de la mort}

\section{Remarques méthodologiques}

Seules les maladies laissant une trace sur le squelette, sont accessibles. En outre la destruction d'une partie des os peut empêcher d'apprécier l'extension des lésions observées. Enfin doivent être distingués lésions pathologiques, variations anatomiques et dégradations osseuses post mortem. Aussi, en dépit de moyens d'investigation de plus en plus efficaces, une grande partie des maladies des populations du passé nous échappe et le diagnostic de celles qui laissent des séquelles sur le squelette, n'est jamais simple à poser.

\section{Échantillon gravettien}

Des pathologies traumatiques ont été décrites sur le squelette de Barma de Caviglione 1 sur lequel Rivière (1887) signale une fracture consolidée du radius gauche. Thillaud (1985) diagnostique une histiocytose $X$ disséminée pour Cro-Magnon 1. Sur Dolni Vestonice 3 est décrit une déformation congénitale du crâne ainsi qu'une atteinte de l'articulation temporo-mandibulaire gauche liée à une patholo-

(6) D’après cette analyse très contestée, S2 serait un garçon, S3 une fille. 
gie chronique. En revanche le squelette infracrânien est indemne de toute lésion ante mortem (Trinkaus et al. 1997). Sur Dolni Vestonice 16, une fracture survenue dans l'enfance pourrait être à l'origine d'une asymétrie faciale (Trinkaus et al. 2006). Divers diagnostics ont été avancés à propos Dolni Vestonice 15 : paralysie du côté droit résultant d'une encéphalite dans le jeune âge (Klima 1987a), séquelles de rachitisme (Jelinek 1992), forme modérée de chondrodysplasie ponctuée $-X$ linked dominant form(Formicola et al. 2001). Trinkaus et al. (2006) admettent que ce jeune adulte souffrait d'une forme de dysplasie congénitale. Ces auteurs évoquent aussi une fracture consolidée de l'extrémité distale de l'ulna et de l'humérus et une série d'anomalies osseuses (asymétrie du frontal, déformation du fémur droit, de l'humérus droit, du radius et de l'ulna gauche, scoliose...) dont les liens avec la dysplasie sont à éclaircir. Pour autant, sans nier un impact possible sur le quotidien de cet individu, ils considèrent que ces problèmes n'ont pas eu de retentissement sur son aptitude locomotrice et sur sa capacité à s'investir dans le groupe. Aucun problème majeur ne semble avoir affecté Dolni Vestonice 14. Selon ces auteurs les traumatismes mineurs observés sur la voûte crânienne de Dolni Vestonice 3, 13, 15,16 et Pavlov 1 relèvent sans doute de chocs peu importants explicables par le mode de vie de ces populations. En revanche, le traumatisme facial surmonté de Dolni Vestonice et le traumatisme crânien de Dolni Vestonice 11/12 (vestige isolé) suggère un impact plus violent dont la cause n'est pas déterminable. Oliva (2000) mentionne un traumatisme crânien surmonté pour Brno 2 . Formicola et al. (2002) décrivent un raccourcissement et une anomalie bilatérale de courbure des fémurs de Soungir 2 en relation avec un diabète maternel.

Formicola (1991) décrit pour les spécimens de Barma Grande et de la grotte des Enfants des lésions dentaires et un petit ostéome au niveau occipital sur le crâne de Barma Grande 4. Mallegni et al. (2000) diagnostiquent pour Veneri Parabita 1 une spondylarthrite ankylosante peu convaincante selon Villotte (2008). Une trépanation est signalée sur un fragment crânien de Kostenki 8 mais on ignore si le sujet a survécu (Sinitsyn 2004). Enfin, ni les squelettes de Baousso da Torre (Henry-Gambier, Villotte en cours d'étude), ni ceux de l'abri Pataud (Nespoulet et al. sous-presse) ne présentent de lésions traumatiques majeures ayant pu entraîner la mort.

Dans ces divers exemples, les lésions ou anomalies dont certaines pouvaient être gênantes ne sont pas à l'origine du décès survenu bien souvent longtemps après.

Par ailleurs, une surmortalité des jeunes femmes liée à la grossesse, à l'accouchement ou à ses suites, a souvent été évoquée comme caractéristique des populations anciennes et en particulier des populations paléolithiques. En réalité cette surmortalité des femmes jeunes dans les populations anciennes résulte d'un biais méthodologique dans la détermination de l'âge individuel au décès à partir du squelette (Masset 2002). II reste que la grossesse et l'accouchement ont effectivement représenté une cause de décès des femmes jusqu'à une date récente dans beaucoup de populations.
La grossesse et/ou l'accouchement comme cause de mortalité des femmes gravettiennes a notamment été discutée à propos des femmes de Cro-Magnon, de l'Abri Pataud et d'Ostuni qui étaient associées à des immature de la classe périnatale. Or, la seule femme identifiée avec certitude (CM 4316) à Cro-Magnon a dépassé 50 ans. Cet âge exclut une cause obstétricale directe ou indirecte de décès (Henry-Gambier et al. 2006). Les âges de la femme (Pataud 1) et de l'immature (Pataud 2) sont compatibles avec une relation mère/enfant et avec un décès lié à la grossesse ou à l'accouchement mais aucun autre argument ne vient l'appuyer. L'âge de la deuxième femme (Pataud 3) trouvée à proximité d'un autre enfant (Pataud 4) est totalement inconnu. Ostuni est le seul exemple où la relation mère/enfant est certaine car un fœtus proche du terme se trouvait à hauteur du bassin de la femme (Ostuni 1) (Vaca et al. 1993). Toutefois, aucun indice ostéologique ne permet d'affirmer que la cause du décès est d'ordre obstétrical.

On sait que la réduction de la mortalité périnatale et infantile est récente et il est très vraisemblable qu'au Gravettien, les premiers instants de la vie et les premières années étaient à haut risque comme dans toutes les populations préjenneriennes. Le nombre non négligeable d'immatures gravettiens décédés avant cinq ans (sites de Pataud, Cro-Magnon, Predmost, Krems, Kostenski, Lagar Velho) témoigne de cette constatation mais les causes possibles de leur mort qui peuvent être multiples ne sont jamais identifiées.

Il est, en réalité, exceptionnel d'élucider les causes du décès à partir du squelette quels que soient l'âge au décès et le sexe : l'échantillon gravettien ne déroge pas à cette règle.

En revanche, il convient de réaffirmer que les indices tels que traces de blessures par arme, traumatismes en faveur de comportements violents, sont inexistants ou rares. Hormis ceux de Dolni Vestonice $3,11 / 12$, les traumatismes observés sur les squelettes et notamment sur le crâne sont la manifestation de chocs peu violents pouvant se produire dans le cadre des activités quotidiennes.

\section{2 - CARACTÉRISTIQUE DES DÉPÔTS}

\section{Dépôt primaire/ dépôt secondaire}

\section{Remarques méthodologiques}

La définition de ces deux notions varie selon les auteurs et la distinction des deux types de dépôt à partir des faits archéologiques est complexe (Boulestin et al. 2005).

Dépôt primaire : II correspond à l'« apport d'un cadavre " frais » dans le lieu de dépôt définitif où va donc s'opérer toute la décomposition du corps; son intégrité est préservée, du moins du point de vue macroscopique " (Duday 2005 - p. 194 ). Le dépôt est effectué peu de temps après le décès sauf cas particulier où le sol gelé empêche une mise en terre immédiate du corps, hypothèse possible pour le Gravettien mais non démontrable. 
La démonstration du caractère primaire repose sur plusieurs critères :

1) maintien des connexions anatomiques (Cr.1A) et en particulier des plus labiles (Cr.1B): colonne cervicale, mains et extrémité distales des pieds, jonction scapulo-thoracique. Ce critère est l'un des plus important ;

2) maintien de l'agencement initial de la parure «assimilable» à la préservation des connexions labiles ;

3) présence de petits os (os des mains, des pieds, os de très jeunes enfants et points d'ossification).

Dépôt secondaire : Il est défini comme le dépôt d'os secs consécutif à une phase de décharnement intervenue dans un autre lieu (Duday 2005). Les critères les plus souvent considérés sont les suivants :

1) désordre des ossements ;

2) absence des petits os, détruits ou oubliés lors des manipulations et/ou de la sélection des ossements ;

3) traces d'action humaine intentionnelle (stries, brûlures...).

La démonstration n'est jamais simple et le contexte doit être discuté. Ainsi, l'assimilation directe d'un assemblage osseux trouvé sans connexion anatomique à un dépôt secondaire intentionnel est une erreur. II faut au préalable exclure d'éventuels remaniements naturels ou anthropiques récents (évolution postérieure au dépôt des sédiments, action des carnivores, travaux récents, ...). De même, l'absence des petits os (Cr.3) n'est pertinente que si toute autre cause (taphonomique ou action anthropique récente telle que non reconnaissance, oubli ou destruction des os lors des fouilles) a été éliminée.

Des traces de brûlures sur les os ne témoignent pas obligatoirement d'un dépôt secondaire, la crémation (si elle est démontrée) ayant pu s'effectuer sur place (Boulestin et al. 2005).

Quant aux traces de découpe, si elles signent une intervention sur le cadavre, leur identification et leur interprétation sont souvent très problématiques dans le cas de vestiges humains paléolithiques.

En fait, le « diagnostic de dépôt secondaire intentionnel repose principalement sur des arguments négatifs " (Duday 2005 - p. 195). Pour cette raison, il est beaucoup plus difficile à poser.

\section{Echantillon gravettien}

Les connexions (Cr.1) de 41 individus (dont 25 avec des connexions labiles) ont été observées au moment de la découverte (tabl.10, 11 et 12 - en annexe). Ces observations indiquent des dépôts primaires.

Le squelette de Pavlov 1, correspond bien à un dépôt primaire. L'absence de certains os, l'orientation des fémurs « parallèles et à l'envers » (extrémités distales du côté des scapulas et du haut du thorax) n'indiquent pas le dépôt secondaire d'un corps incomplet, préalablement désarticulé comme l'affirme M. Oliva (2000-2001). Les fémurs «ne sont pas à l'envers » mais leur position correspond à celle d'un corps placé sur le côté droit, membres inférieurs hyper fléchis (genoux, donc extrémité distale des fémurs vers le haut du corps). Une connexion lâche entre humérus et ulna, plaide aussi en faveur d'un dépôt primaire remanié. Ici, le glissement des os, la dislocation partielle des connexions et la disparition d'une partie des os sont sans doute imputables aux phénomènes de solifluxion qui ont affecté les niveaux archéologiques (Klima 1987 a).

En revanche, on ne connait pas de connexions anatomiques pour Cro-Magnon 1 à 5 , cinq des individus de Pataud, Cussac locus 1 et locus 3, le Figuier 1, Brno 2, Kostenki 8 et 15 , les vestiges fragmentaires des Marronniers, de Paglicci, de Pavlov I, de Dolni Vestonice I et II.

La présence à Cro-Magnon et au Figuier de petits os (Cr. 3) des mains et des pieds ou celle d'os et de points d'ossification d'immatures, sont cependant en faveur de dépôts primaires, perturbés anciennement et lors de la mise au jour.

À Pataud, la situation est complexe car les agents ayant entraîné la dislocation de l'essentiel des connexions anatomiques et la disparition d'une partie des ossements ne sont pas identifiés. Les études en cours ne permettent pas d'exclure des manipulations secondaires.

Quant à Cussac, l'étude reste à faire !

La dislocation des connexions anatomiques et l'absence d'une grande partie des os de Brno 2 (Oliva 2000) n'impliquent pas non plus un dépôt secondaire. Les conditions d'exhumation (découverte fortuite par des ouvriers) sont vraisemblablement en partie à l'origine des désordres et des destructions les plus importants.

Les vestiges fragmentaires et erratiques des secteurs de Pavlov I et Dolni Vestonice I et II sont constitués de portions de crânes, de dents isolées et de rares os du squelette infracrânien d'adultes et d'immatures. Les vestiges de Dolni Vestonice II sont les seuls ayant fait l'objet d'une approche taphonomique (Trinkaus et al. 2000). Les os n'ont pas de stigmates imputables aux carnivores. Ils sont érodés et dégradés par des racines. Ils présentent des stries de charriage (DV 11) et des cassures anciennes dont la forme évoquent une fracturation sur os sec. Les rares informations relatives aux vestiges des sites de Dolni Vestonice I et Pavlov I, indiquent un même type de modifications. L'hypothèse explicative la plus parcimonieuse est celle de dépôts primaires perturbés voire en grande partie détruits par des processus naturels (solifluxion notamment). S'y sont peut-être ajoutés des remaniements anciens d'origine anthropique consécutifs à l'aménagement des espaces lors des occupations successives des sites. L'association de canines percées de renard polaire à Dolni Vestonice 4 (secteur Dolni Vestonice I), un squelette très partiel d'enfant, trouvé par Absolon en 1927 sous une scapula de mammouth, confirmerait par ailleurs cette interprétation. 
Cette dernière pourrait s'appliquer aux vestiges isolés et fragmentaires de Paglicci et de Soungir. Des analyses de leur état de surface et du contexte archéologique seraient cependant nécessaires pour conclure définitivement.

L'adulte de Kostenki 8, est le seul exemple annoncé impliquant directement le feu ${ }^{7}$. II pourrait correspondre à un dépôt secondaire après crémation. Toutefois, les données sont trop succinctes pour exclure un dépôt primaire remanié dont les constituants auraient été mis fortuitement au contact du feu.

L'hypothèse d'un dépôt secondaire (Onoratini et al. 1996) à propos des restes du site du Marronnier n'est pas défendable à partir des données disponibles.

À Paviland est évoqué un possible prélèvement du crâne par les préhistoriques (Aldhouse-Green 2000 b). II n'y a cependant, aucun argument pour supposer que les causes naturelles qui ont abouti à la représentation partielle du squelette infracrânien, ne s'appliquent pas au bloc crânio-facial.

Enfin, une dernière série de remarques concernent les vestiges de Predmost. II n'existe aucun plan d'ensemble des vestiges humains. Sur les esquisses du journal de Maska (In : Svoboda 2008 a et b) l'un des principaux découvreurs, il n'y a pas de connexion anatomique. Toutefois les os de certains individus (ex. Predmost 3) apparaissent regroupés. L'état de conservation des squelettes semble avoir été très variable. Pour autant, il est impossible de suivre $M$. Oliva (2000-2001) qui interprète l'assemblage de Predmost comme des dépôts intentionnels secondaires avec sélection d'ossements. Les inventaires des os (Oliva 2000-2001; Veleminska et al. 2008) font état d'os des mains et des pieds. Les os manquants sont les plus fragiles ou les plus difficiles à identifier (os d'immatures par ex.). En outre, le taux de représentation des squelettes est analogue à celui de nombreux dépôts dont le caractère primaire n'est pas contestable. L'hypothèse d'un dépôt secondaire avec décharnement préalable ne peut non plus être étayée par les traces de sciage, de fractures volontaires ou les stries décrites par Ullrich (1997) sur des photographies de Matiegka (1934-1938). Oliva attribue d'ailleurs à la spatule des archéologues les traces sur le fémur $\mathrm{XXVII}{ }^{8}$, conservé à Brno. Cette remarque montre qu'un retour aux fossiles originaux serait nécessaire. Il est malheureusement impossible car la presque totalité des vestiges a été détruite. L'analyse du journal de Maska révèle que la fouille a été assez chaotique (Oliva 2000-2001; Svoboda 2008 a et b). Une partie du désordre des ossements et des absences est sans doute imputable à la méthode de fouille peu favo- rable à des observations in situ fiables. Aussi, la conservation différentielle, la destruction et la sélection des os lors des fouilles suffisent à expliquer les caractéristiques de l'assemblage. Les données sont par conséquent en faveur de dépôts primaires perturbés.

L'analyse de l'échantillon gravettien montre que quelle que soit la région, il s'agit de dépôts intentionnels primaires.

Les vestiges fragmentaires et dispersés comme ceux de Pavlov I (2- 28), Dolni Vestonice I (1-2 ; 4-10 ; 23-32 ; 35 ; 37-38), Dolni Vestonice II $(11,12,17,33,34,36,39,40$ à $53)$, résultent plus vraisemblablement de bouleversements d'origine naturelle et/ou anthropique anciens (sans lien avec les pratiques funéraires) au cours des occupations ultérieures.

Les vestiges de Paglicci (A, B, C, D, F, G, H, I,, J, L, M, N), des Marronniers, de Kostenki 8, de Soungir 4 à 9 et de La Rochette 1-2 demeurent « indéterminés ». Quant aux vestiges de Pataud, l'étude en cours permettra peut-être de comprendre l'originalité de cet assemblage.

\section{Dépôts individuels/ multiples/ collectifs}

\section{Remarques méthodologiques}

L'identification d'un dépôt individuel est simple mais dès lors que plusieurs individus sont regroupés dans un même espace (sépultures double, triple, multiple et collective ${ }^{9}$ ), l'analyse devient complexe que l'on considère le dénombrement en l'absence de connexion anatomiques (cf. supra) ou le fait qu'un nouvel élément s'ajoute : la chronologie des dépôts. Les données ostéologiques constituent un élément majeur de la discussion sur ce dernier point. Si l'intervalle de temps entre les dépôts est important, l'introduction d'un autre cadavre doit induire des remaniements du ou des squelettes déposés en premier et une destruction des contentions articulaires. Les articulations labiles sont les plus pertinentes pour en juger. II n'est cependant pas possible de différencier les dépôts lorsque le délai les séparant est inférieur au temps nécessaire à la dislocation des articulations (Duday et al. 1990 ; Duday 2005).

\section{Échantillon gravettien}

Le Gravettien a livré 30 dépôts individuels, quatre dépôts doubles dont un en Russie ${ }^{10}$ (Soungir 2, 3) et deux en Italie (Veneri Parabita 1, 2 et Grotte des Enfants 5, 6), un en Autriche (Krems A, B); deux dépôts triples dont un en République Tchèque et un en Italie (Dolni Vestonice 13, 14, 15 et Barma Grande 2, 3, 4). Predmost, Cro-Magnon et

(7) L'hypothèse d'une crémation sur place de Barma Grande 6 a été réfutée par Shipman et al. (1984).

(8) Il s'agit de l'os figuré (fig.b p. 26) et qualifié d'humérus (Svoboda 2008 b). Il s'agit bien d'un fémur.

(9) Multiple correspond au dépôt simultané de plusieurs sujets, collectif au dépôt échelonné dans le temps de plusieurs sujets (Leclerc et al. 1988).

(10) lakovleva et al. 2004, mentionnent 2 immatures dans la sépulture de Malt'a. Cette information est à contrôler. 
Pataud sont cités comme exemples de dépôt multiples ou collectifs. S'y ajoute depuis 2000, le locus 3 de Cussac avec au moins trois individus.

\section{Les sépultures doubles}

A Soungir, les deux squelettes d'enfant opposés par la tête n'étaient pas en contact (Alexeeva et al. 2000). La simultanéité des dépôts est fondée notamment sur la position des deux grandes lances d'ivoire qui dépassent l'emprise de chaque corps.

Dans la grotte des Enfants, la femme âgée (GE5), introduite en second dans la fosse, était au-dessus de l'adolescent (GE6). Bien que le squelette de la femme soit en position très contractée et « tassé » comme si la fosse avait été trop petite pour contenir deux corps, il n'y a pas d'argument ostéologique pour réfuter la simultanéité des dépôts. La dispersion des éléments de parure autour du crâne de l'adolescent est plutôt à mettre en relation avec la présence d'un espace vide autour du crâne, espace ménagé par des pierres selon Verneau (1906).

À Veneri Parabita, les remaniements anciens mais postérieurs au Paléolithique n'ont pas permis d'observer l'état des connexions labiles mais les connexions de la moitié inférieure des deux squelettes étaient préservées (Cremonesi et al. 1972 ; Henry-Gambier 2001).

\section{Les sépulture triples}

À Barma Grande, les informations sont moins fiables mais les squelettes ne semblent pas avoir subi de perturbations majeures autres que celles liées à leur exhumation mouvementée (Verneau 1906 ; Henry-Gambier 2001, 2005). L'étude n'est pas achevée, mais il est très vraisemblable que les deux enfants de Krems ont été déposés en même temps.

À Dolni Vestonice, les sujets latéraux (DV13 et 14) s'appuyaient en partie sur le sujet central (DV 15) déposé en premier. L'ajout de Dolni Vestonice 13 et 14 n'a pas perturbé le squelette central (Jelinek 1992). Les désordres au niveau des pieds sont à mettre en relation avec des phénomènes postdépositionnels (Svoboda 2008 a).

\section{Les dépôts multiples ou collectifs}

Pour Predmost, l'image véhiculée à la suite des fouilles de Maska est celle de plusieurs adultes, adolescents et enfants gisant en position fléchie dans une fosse « fermée " par des scapulas de mammouth. Oliva (2000-2001) et Svoboda (2008 $a$ et b) ont montré que cette reconstitution était erronée. L'analyse des carnets de fouille de Maska indique que les individus les mieux conservés gisaient sur une surface d'environ $10 \mathrm{~m}^{2}$. Des vestiges plus fragmentaires étaient dispersés autour. Dans la concentration, les enregistrements de Maska ne permettent pas de statuer sur la chronologie des dépôts. Svoboda (2008 a et b) retient l'hypothèse de dépôts successifs dans une fosse dont la faible profondeur explique l'impact fort des processus postdépositionnels et des perturbations induites par la poursuite de l'activité des hommes sur le site. Il évoque aussi le rôle des renards et des loups en dépit de l'absence de traces de rongement. En l'état des informations, il est impossible de se prononcer sur le nombre initial de corps, leur regroupement et la chronologie des dépôts. L'image d'une fosse commune paraît cependant très contestable. En effet, les archives indiquent une association possible de scapula de mammouth à certains individus. Ces faits seraient en faveur de dépôts individuels analogues à ceux de Dolni Vestonice et de Pavlov.

A Pataud, deux concentrations (l'une avec connexion) livrant chacune au moins deux individus étaient distantes d'environ huit mètres (Nespoulet et al. sous-presse) Entre ces deux concentrations, les restes d'au moins deux autres sujets étaient dispersés. Doit- on parler de dépôts doubles? d'un dépôt multiple? d'un dépôt collectif dans un espace perçu comme unique par les préhistoriques ? Les révisions en cours permettront peut-être de répondre à ces questions.

A Cro-Magnon, il est impossible de trancher entre dépôt multiple et collectif car il n'existe aucun plan détaillé montrant les relations entre les os.

Quant à Cussac, l'absence de traces dues aux carnivores ainsi que le taux de représentation des ossements déposés en surface, indiquent que la cavité était inaccessible aux carnivores. Mais nous ignorons si la grotte a fonctionné comme lieu de dépôt multiple (fermé après le dépôt simultané de tous les défunts) ou collectif (fermée après chaque dépôt). Des études géologiques et anthropologiques sont nécessaire pour répondre à ces interrogations.

Le nombre de sépultures primaires multiples indiscutables est ainsi de 6 pour un total de 30 sépultures individuelles primaires. La chronologie des dépôts des sites de Predmost, Cro-Magnon, Pataud et Cussac est en revanche non résolue.

\section{Position initiale du corps / position du squelette}

\section{Remarques méthodologiques}

Il existe des différences entre la position initiale du corps et celle du squelette observé à la fouille. La disparition des parties molles en raison de la décomposition du cadavre, a pour conséquence la création d'espaces vides dans lesquels les os peuvent se déplacer sous l'action de la gravité lorsque les contentions ligamentaires cèdent : mise à plat de la cage thoracique pouvant entraîner la chute du sternum ou des os des mains si elles reposaient sur la cage thoracique; dislocation partielle de la colonne vertébrale qui apparaît segmentée en sous-ensembles; chute des os des mains lorsqu'elles sont placées sur l'abdomen, ouverture du bassin. La distorsion entre position initiale et position observée à la fouille peut aussi concerner la tête. Lorsqu'il y a un espace vide, la position de la tête sur un côté ou son basculement en arrière peut découler d'un mouvement au cours de la décomposition du corps alors qu'elle se trouvait initialement en position axiale (Duday et al. 1990). 
Ces remarques sont encore peu prises en considération dans l'analyse des tombes paléolithiques.

\section{Echantillon gravettien}

À l'échelle de l'Europe, la position publiée des corps quels que soient l'âge et le sexe apparaît assez variable surtout si l'on prends en considération les membres supérieurs (tab. 5, 6, 7 - en annexe).

1 - corps en position contractée sur le côté droit ou gauche, membres supérieurs fléchis, membres inférieurs fléchis, pieds sous les fesses (Pavlov 1, Dolni Vestonice 3, Dolni Vestonice 16, Kostenki 14, 18, Malta 1) ;

2 - corps en position contractée, moitié supérieure du corps sur le dos ou sur le ventre, membres supérieurs fléchis, membres inférieurs repliés sur la droite ou sur la gauche, pieds sous les fesses. (Grotte des enfants 5, 6, Ostuni 1 et 2) ;

3 - corps sur le côté gauche, membres supérieurs fléchis main à hauteur du cou, membres inférieurs à peine fléchis (Barma Grande 3, 4, Barma de Caviglione 1, Baousso da Torre 2) ;

4 - corps allongé sur le dos, membres inférieurs étendus, un ou deux membres supérieurs replié (s) sur le thorax (mains sous le cou ou ramenées sur le pubis) ou de part et d'autre du corps (Arene Candide I, Barma Grande 5, Barma Grande 2, Baousso da Torre 1, Grotte des Enfants 4, Paglicci 15, 25, Lagar Velho 1, Kostenki 12, Soungir 1, $2,3)$;

5 - corps sur le ventre, position des membres inconnue (Baousso da Torre 3, Cussac locus 2) ;

6 - position assise (Kostenki 15 et 2).

L'agencement des défunts de la sépulture triple de Dolni Vestonice est singulière: les membres supérieurs de Dolni Vestonice 13 (allongé sur le dos) sont tendus en direction de Dolni Vestonice 15, main gauche reposant sur le pubis de ce dernier. Dolni Vestonice 15 est sur le dos. Dolni Vestonice 14 placé sur le ventre, " regarde » vers l'extérieur, le coude gauche appuyé sur Dolni Vestonice 15, le membre supérieur droit à l'extérieur du corps est en légère flexion.

Lorsque les corps sont sur le côté droit, le crâne apparaît par sa face latérale gauche, lorsqu'ils sont en flexion sur le côté gauche, le crâne apparaît par sa face latérale droite. Lorsque les corps sont sur le dos ou sur le ventre, la tête est soit en position axiale, (visible par la face antéro-supérieure ou par la face postérieure du crâne), soit en rotation sur le côté. Dans ce dernier cas, en l'absence de données sur la liaison bloc cranio-facial / rachis cervical, on ne peut affirmer qu'il s'agit de la position initiale (tabl. 13, 14 et 15 - en annexe).

La position initiale de nombreux défunts (Cro-Magnon 1 à 5, Pataud 1 à 6, Barma Grande 6, Barma Grande 1, Cussac locus 1 et locus 3, Brno 2, Veneri Parabita 1, 2, Predmost) est imprécise ou inconnue. La localisation des concrétions sur les os coxaux $\left(n^{\circ} 4314\right)$ de Cro-Magnon 1 , suggère cependant un corps placé sur le côté. La position des membres supérieurs de la femme de la bande II de l'abri Pataud est compatible avec un corps en flexion sur le côté gauche, membres supérieurs fléchis, mains à hauteur du bloc cranio-facial. Les données sur les os des membres inférieurs de Barma Grande 6 plaident en faveur d'une position contractée. Veneri Parabita 1 et 2 étaient vraisemblablement sur le dos, membres inférieurs étendus. Buckland (1823), représente le squelette de Paviland sur le dos, le genou gauche surélevé. La disparition de la moitié droite du squelette suggère un décubitus latéral gauche favorisant la destruction de la moitié droite en position plus superficielle.

\section{Fosses, éléments architecturaux, décomposition en espace vide ou colmaté}

\section{Remarques méthodologiques}

La reconnaissance d'une fosse ou d'élément tels qu'os ou pierre participant à la tombe comme les conditions de décomposition sont des données étroitement dépendantes de l'évolution postdépositionnelle et des méthodes de fouilles. Dans ces conditions l'absence de fosse peut résulter d'un défaut d'observation au moment de la découverte.

Les observations ostéologiques apportent aussi des éléments sur l'architecture funéraire et permettre de déterminer dans quelles conditions, espace vide ou colmaté, le corps a évolué. Si la décomposition du cadavre s'est effectuée dans un espace vide, des os peuvent sortir du volume initial du corps sous l'effet de la pesanteur. Ces mouvements quand ils sont dus à la gravité dépendent de la position originelle du corps. Les os susceptibles de tomber hors du volume initial du corps sont ceux que la décomposition libère en situation de déséquilibre par rapport à celui -ci (Duday et al. 1990 ; Duday 2005). La mise à plat des os coxaux lorsque le sujet est sur le dos, la rotation latérale des fémurs et la chute des patellas en dehors constituent des indices d'une décomposition en espace vide. Au contraire, si les os ne tombent pas, le volume n'est pas assimilable à un espace vide. On parle alors d'effet de paroi dont il faut trouver la nature : limite de fosse ou élément périssable situé à l'intérieur de celle-ci et plus fréquemment le sédiment utilisé pour combler la fosse.

\section{Échantillon gravettien}

Hormis à Cussac, tous les squelettes étaient enfouis au moment de la découverte et les données ostéologiques disponibles, plaident dans la majorité des cas en faveur d'une décomposition en espace colmaté mais des incertitudes persistent.

Dans la majorité des cas, le dispositif se limitait à une fosse de forme et de dimensions variables. Vingt-sept individus étaient dans une fosse d'origine anthropique souvent ajustée $\mathrm{au}(\mathrm{x})$ corps. Huit (Cussac 1 à 5 , Le Figuier 1, Veneri Parabita 1,2) reposaient dans des fosses naturelles : bauges d'ours à Cussac, encorbellement de blocs au Figuier, dépression du socle à Veneri Parabita (tab. 5, 6, 7 - en annexe). Svoboda (2008 a et b) souligne la faible profondeur des fosses en République Tchèque qu'il explique par la difficulté à creuser le sol gelé. 
A Barma de Caviglione et Baousso da Torre, Rivière (1887) défend l'absence de fosse et indique que les 4 défunts déposés sur le sol avaient été recouverts superficiellement. Les désordres des squelettes de Baousso da Torre décrits par Rivière ainsi que les traces de rongement par des animaux (carnivores et ou rongeurs) sur les os plaident en faveur de dépôts accessibles. II est donc possible que Baousso da Torre 1, 2, 3 n'aient été que très superficiellement enfouis. Rivière était convaincu que Barma de Caviglione 1 était mort pendant son sommeil et avait été abandonné sur place. Cette idée préconçue a peut-être favorisé la non-reconnaissance des limites d'une fosse. Quoi qu'il en soit, l'état remarquable de conservation, le maintien de toutes les connexions de $\mathrm{BC} 1$ indiquent que le corps avait reçu une protection efficace.

Paglicci 15 n'était pas dans une fosse.

Pour Paviland 1, Cro-Magnon 1-5, Pataud 1-6, Barma Grande 1, 5, 6, Grotte des enfants 4, Pavlov 1, Brno 2, Kostenki 12, 8 , les informations sont très précaires et il est difficile de conclure définitivement.

Dans plusieurs sépultures, des éléments architecturaux (pierres, scapulas parfois gravées de mammouth, os, etc.) sont mentionnés. En Europe occidentale, des pierres sont signalées sous/autour/au-dessus du corps parfois autour de la tête. Un " caisson " vide de sédiment est parfois mentionné autour du crâne de GE6 (Verneau 1906).

La faible qualité des fouilles anciennes et/ou la fréquence élevée des blocs dans les niveaux archéologiques jettent un doute sur leur appartenance au dispositif funéraire.

En Europe orientale et centrale (Russie et République Tchèque, Autriche), l'utilisation d'os de mammouth est bien attestée. Toutefois, à Kostenki, les sépultures recoupent ou sont recoupées par des « cabanes » ou par des accumulations d'os de mammouth de telle sorte que la relation entre os de mammouth et sépulture n'est pas toujours claire (Sinitsyne 2004). À Dolni Vestonice, une structure en bois matérialisée par quelques fragments de bois épars protégeait peut-être les défunts de la triple sépulture (Klima 1987 a ; Jelinek 1992).

Duarte (2002) mentionne un linceul semi-rigide en peau autour de l'enfant de Lagar Velho pour expliquer I'hyper extension post mortem du pied droit et la préservation des connexions anatomiques, préservation impossible si du sédiment avait compressé le corps. D'après les relevés de fouille (Duarte 2002), le corps placé sur le dos dans une fosse, était en légère rotation vers la gauche, membres supérieurs en extension de part et d'autre du corps. Il y a mise à plat du gril costal. L'aile iliaque gauche est à plat, la droite, mieux conservée, est légèrement redressée (effet de paroi lié à la remontée du fond de la fosse) et la liaison avec le sacrum est maintenue. Les os des membres inférieurs sont en connexion et les points d'ossification sont en position anatomique. Ces caractéristiques plaident en faveur d'une décomposition en espace colmaté. Le pied droit est effectivement en exten- sion mais disloqué au niveau de l'articulation médio tarsienne. Les métatarsiens et les phalanges sont à la même altitude que le tarse. Cette disposition est effectivement en faveur d'un espace vide autour des pieds. Mais rien ne permet de démontrer que cet espace vide s'étendait à tout le corps. La répartition de l'ocre ne prouve pas non plus la présence d'un linceul. Un dépôt d'ocre sur le corps pourrait tout aussi bien donner la même image, l'ocre migrant au cours de la décomposition du corps au fond de la fosse en respectant le contour de celui-ci.

Dans le cas de Kostenski 15, assis dans une fosse, l'affaissement des segments supérieurs du squelette plaide en faveur d'une décomposition en espace vide, mais un relevé détaillé de la disposition des os serait nécessaire pour conclure définitivement.

Enfin, à plusieurs reprises des auteurs (Oliva 2000-2001 ; Sinitsyn 2004), mentionnent des processus de décharnement avant le dépôt des corps ou des liens pour expliquer la position " très contractée» de certains défunts (Dolni Vestonice 3, Kostenski 14, ...). En fait, lorsque le défunt repose en pleine terre, dans une attitude contractée, la pression périphérique des sédiments sur le corps peut induire une fermeture progressive des angles inter - segmentaires (Duday et al. 1990). Là encore, une étude soigneuse de la surface des os serait souhaitable pour rechercher d'éventuelles traces anthropiques d'intervention sur le cadavre.

\section{Mobilier et parure}

\section{Remarques méthodologiques}

L'analyse du mobilier et de la parure se heurte à plusieurs difficultés que les découvertes soient anciennes ou récentes. D'une part, les constituants classiques du mobilier et de la parure (os de faune, coquillages et dents percés, outils, minéraux...) sont loin d'être rares dans les remplissages archéologiques en dehors de tout dépôt de cadavre aussi leur association intentionnelle aux vestiges humains n'est pas toujours démontrable (tabl. 14, 15, 16 en annexe). D'autre part, comme les os, les éléments de parure ou de mobilier peuvent être déplacés lors de la décomposition du corps, la restitution de leur agencement initial passe par la compréhension des mécanismes de décomposition du cadavre (Duday et al. 1990 ; Duday 2005).

Cela signifie que le classement en résille, diadème, collier ou bracelet, fondé sur la localisation à la fouille des éléments de parure n'est acceptable que lorsqu'il est étayé par des observations précises sur leur agencement et leur relation avec les os et discuté en tenant compte des processus de décomposition du corps.

\section{Echantillon gravettien}

\section{$\underline{\text { Parure }}$}

À Cro-Magnon, à Paviland, au Figuier, à Barma Grande, à la grotte des Enfants, à Veneri Parabita les conditions 
de découvertes et/ou les remaniements postdépositionnels excluent toute conclusion sérieuse sur les relations précises entre éléments de parure et corps. À Lagar Velho, la disposition des canines de cervidés en diadème frontal (Zilhao et al. 2002) est une hypothèse totalement spéculative puisque déduite de leur seule proximité avec des fragments crâniens dispersés à la suite de travaux récents.

Quelques individus -Barma de Caviglione 1, Baousso da Torre 1, 2, Arene Candide 1, Paglicci 15 et 25, Ostuni 1, 2 Soungir 1, 2, 3, Kostenki 15, Malta 1... dont la parure avait, semble-il, conservé sa position initiale, indiquent que les sites privilégiés sont la tête, le thorax, les membres supérieurs. II est cependant difficile de séparer les éléments appartenant au décor du vêtement, d'éventuels bijoux. Soungir 1, 2, 3 appartiennent aux rares exemples où des cercles d'ivoire autour des poignets, des coudes ou des doigts seraient interprétables comme des bracelets et des bagues. Dans la majorité des cas, la répartition de la parure évoque un décor du vêtement plutôt que des bijoux.

\section{Mobilier}

Constitué d'outils de silex, d'armes en ivoire, bois de cervidés, d'os travaillés, de galets, ou de minéraux, sa présence est indiscutable sur et autour d'Arene Candide I, Ostuni 1 et 2, Paglicci 15 et 25, Brno 2, Kostenki 15, Soungir 2, 3, Malta 1 (tab. 14,15,16 - en annexe).

Elle est vraisemblable à Barma Grande et à la grotte des Enfants (Barma Grande 1, 2, 3, 4; Grotte des enfants 5 et $6)$, indémontrable dans les autres exemples.

Très abondant à Brno, Soungir (Soungir 1 et 2), Mal'ta, le mobilier comprenait en outre des objets d'art en ivoire.

Baousso da Torre 3, Kostenki 2, 12, 14, 18 ainsi que les sujets de Predmost étaient dépourvus de parure et de mobilier (tabl. 16, 17 et 18 - en annexe). Cette absence pourrait dans bien des cas être liée aux perturbations postdépositionnelles. À Cussac, les observations préliminaires ne révèlent pas d'objets autour des os.

\section{Os de faune}

Des os de faunes non manufacturés sont signalés à plusieurs reprises. C'est sans doute l'élément le plus problématique des tombes paléolithiques. En effet, l'association intentionnelle d'os de faune aux vestiges humains est en général indémontrable parce que leur rapport avec le corps n'a pas été enregistré avec précision et surtout parce qu'il s'agit de vestiges fréquents dans les niveaux archéologiques. L'argument selon lequel la coloration par l'ocre de ces os validerait l'association n'est pas recevable car l'ensemble des objets contenus dans le sédiment entourant le défunt peut être secondairement imprégné par l'ocre répandue sur le corps.
L'un des seuls exemples probant est la tombe d'Arene Candide 1 où des os de queue d'écureuil ont été trouvés. Mais il s'agit plutôt d'ornements du vêtement.

\section{Le cas des scapulas de mammouth.}

Trouvées au sommet de certaines tombes d'Europe orientale et centrale, il est difficile de déterminer leur fonction dans le dispositif funéraire en particulier pour celles qui sont gravées. Elles pourraient à la fois avoir un rôle de protection du dépôt ou de signalement mais aussi avoir une signification symbolique et à ce titre, appartenir au mobilier.

\section{L'ocre}

Fréquemment annoncée, son abondance et sa répartition (tête, abdomen, totalité du corps...) sont variables. Les sépultures italiennes sont particulièrement " riches " en ocre. Certains squelettes en sont totalement dépourvus (tabl. 5, 6 et 7 - en annexe). Sa persistance sous forme d'encroûtement d'épaisseur importante comme sur le crâne de Baousso da Torre 2, ou sous forme d'une coloration rosée des os (Cro-Magnon 1 à 4, Pataud 1, Baousso da Torre 1) permet de valider des observations anciennes. En revanche, le sillon rempli d'ocre mentionné par Rivière (1887) à hauteur de l'orifice nasal et de la bouche de Barma de Caviglione 1 reste unique et hypothétique.

Quelle signification attribuer à ce pigment qui accompagne des défunts des deux sexes et de tout âge ? Est-on sûr qu'il est lié au contexte funéraire ? L'interrogation est légitime : l'usage de peintures corporelles est fréquent dans de nombreuses populations, il est donc possible que l'ocre soit l'apanage des vivants et non celui des morts. S'il relève du dispositif funéraire s'inscrit-il dans un cadre symbolique ou fonctionnel ? Ces questions ont été débattues à de multiples reprises. Peu d'études récentes sur la composition et l'origine de ce colorant selon le contexte (funéraire, habitat, grottes ornées) ont été réalisées (Aldhouse-Green $2000 \mathrm{~b}$; Young 2000). II y a là, un champ de recherche utile à développer pour espérer dépasser le stade des questions.

\section{3 - SYNTHÈSE ET DISCUSSION}

L'analyse de la documentation anthropologique révèle dès le début du Gravettien des comportements nouveaux pour le Paléolithique supérieur européen. Le sort des défunts de l'Aurignacien est en effet totalement inconnu. Entre 35000 BP et $27000 \mathrm{BP}$, la seule intervention démontrée sur le cadavre est le façonnage de dents isolées. Sa mise en relation avec un traitement funéraire est incertaine (HenryGambier et al. 2002, 2004, 2006). Ce type d'intervention est aussi connu dans des sites gravettiens (Abri Pataud, grotte des Vachons en France, Dolni Vestonice et Pavlov ${ }^{11}$ en République Tchèque), mais il est marginal.

À partir de 28-27 $000 \mathrm{BP}$, les gestes mis en évidence autour du cadavre préservent l'intégrité du corps et du squelette. L'analyse ostéologique montre que la sépulture

(11) Deux dents, DV 8 et PV 25, sont signalées par Svoboda (in Trinkaus et al. 2006). 
primaire est, sinon la seule pratique avérée à travers toute l'Europe, en tout cas la seule véritablement attestée et bien documentée. II n'existe actuellement aucun exemple définitivement prouvé de dépôt secondaire intentionnel et/ou de prélèvement partiel et intentionnel de certains éléments anatomiques (hormis les trois dents isolées à racine percée ou travaillée). Les vestiges très fragmentaires et/ou isolés ou les assemblages en désordre (Dolni Vestonice, Pavlov, Predmost, Soungir, Paglicci) résultent plus certainement de remaniements anciens ou récents de sépultures primaires.

Hormis à Cussac, à Kostenki Zamiatnin et peut-être à Baousso da Torre, les corps, déposés dans des fosses ou en surface, ont été recouverts immédiatement.

La présence de linceul ou de liens décrite pour certains, n'est pas vérifiée par des observations ostéologiques. Le dispositif funéraire est simple et limité au creusement d'une fosse ou à l'utilisation d'une dépression naturelle. II est parfois complété par des pierres en Europe occidentale ou par des scapulas de mammouth dont certaines sont gravées en Europe centrale et orientale. La fonction de ces pierres et de ces os est problématique : protection du corps, marquage du lieu destiné à en perpétuer le souvenir, offrandes...?

Les sépultures individuelles dominent et concernent aussi bien des adultes des deux sexes que des immatures du stade périnatal à l'adolescence. La présence de plusieurs individus dans un même espace n'est cependant pas rare mais il n'y a pas d'association type : des adultes des deux sexes et des enfants de tout âge peuvent alors être réunis.

Les défunts, placés dans des positions variées, sont vêtus. Les perles, les dents d'animaux percées, les coquilles, les pendeloques, décorations du vêtement ou éléments de bijoux, ainsi que l'ocre, sont très fréquentes mais elles ne sont pas systématiques. En outre, leur abondance varie. L'importance et la composition du mobilier (outils, œuvres d'art...) bien avérées dans plusieurs cas, sont aussi variables.

L'inhumation de cadavres correspond à des gestes pratiqués dès le Paléolithique moyen en Europe et au MoyenOrient par Homo sapiens neanderthalensis et Homo sapiens sapiens (Tillier 1995). En revanche, l'ocre, le mobilier et les parures répartis autour ou sur le corps constituent des innovations observées seulement à partir du Gravettien.

Toutefois, ce fond commun ne doit pas masquer une réelle diversité qui ne surprend pas si l'on considère les territoires fréquentés et la durée du Gravettien.

Un premier ensemble de remarques peut-être formulé :

1- À l'échelle de l'Europe ou de chaque site, le petit nombre d'individus inhumés suggère une sélection et l'existence d'autres pratiques de gestion des morts moins propices à la conservation et/ou à la découverte des sque- lettes. Cette conclusion n'exclut cependant pas comme facteur explicatif des destructions anciennes ou récentes ou des lacunes des recherches. La détermination des critères de cette sélection se heurte cependant aux caractéristiques de l'échantillon.

Quelle que soit la région, il est en effet difficile de mettre en évidence un recrutement privilégiant un sexe (le sexe masculin), car la proportion des « indéterminés " de tout âge est importante (tabl. 19, 20, 21, 22 - en annexe). En revanche, l'âge au décès paraît plus discriminant, en tout cas dans certaines régions d'Europe. Le pourcentage d'immatures est inégal selon les sites mais dans tous, les classes d'âge les plus jeunes sont insuffisamment représentées (tabl. 19, 21 - en annexe) au regard de ce que l'on sait du schéma de mortalité dans ce type de population. Aussi, bien que des facteurs naturels ou anthropiques de destruction ne puissent, une fois encore, être entièrement rejetés, cela suggère une sélection sur l'âge. Elle est particulièrement nette en Italie où l'absence des plus jeunes enfants est systématique y compris dans des sites fouillés récemment. Elle est possible dans les sites tchèques de la colline de Pavlov où ils ne sont représentés que sous forme de vestiges très fragmentaires et dispersés. Enfin, les trois immatures de Krems en Autriche représentent un autre type d'indice en faveur d'un traitement particulier. Découverts, groupés, ils valident l'idée d'un espace « réservé " aux plus jeunes défunts. Krems constituerait alors un exemple de mise à l'écart de la classe 0-1 an. Ce type de pratique est connue dans de nombreuses sociétés : si un enfant meurt avant que son existence sociale soit reconnue, son traitement funéraire n'est pas identique à celui des adultes (Morin 1970 ; Thomas 1980).

Le rôle de chaque individu dans le groupe n'est pas connu. $\mathrm{Ni}$ le mobilier, ni les caractéristiques biologiques ne permettent en effet d'approcher les activités individuelles. Dans ce registre, l'interprétation (Oliva 2000) de la sépulture de Brno comme étant celle d'un chaman est totalement spéculative. Celle de Mussi et al. (1989), selon laquelle le recrutement en Italie des inhumés autour de 25000 BP aurait obéi à des règles privilégiant les adultes et les adolescents de sexe masculin en raison de leur aptitude à la chasse, n'est que plausible. Si la chasse imprègne fortement la vie sociale des peuples de chasseurs, d'autres activités sont tout aussi fondamentales à l'existence et à la cohésion du groupe ainsi qu'à son développement (Testart 1985). La seule chose que l'on puisse dire est qu'en Italie les sépultures gravettiennes contenaient des individus suffisamment " âgés 》 pour être a priori capables de participer aux diverses activités d'un groupe de chasseurs-collecteurs.

La même remarque s'applique aux sépultures d'adultes et d'adolescents des autres régions.

Autre question à soulever : doit-on considérer le petit nombre de sépultures, comme la preuve d'une hiérarchisation des sociétés gravettiennes, les inhumés appartenant à un groupe social privilégié ? En l'absence de toute connaissance sur les autres dispositifs de gestion des 
morts, il est impossible de répondre, sauf si l'on attribue une valeur particulière à l'inhumation.

Les causes de la mort ne peuvent non plus être invoquées pour discuter cette sélection, puisque nous les ignorons. L'hypothèse d'un recrutement privilégiant les sujets atteints de pathologie est intéressante (Formicola et al. 2001), mais il faut rappeler que les pathologies décrites sont finalement rares et peu importantes dans l'échantillon. De plus, elles ne font pas l'unanimité.

2- À quoi renvoient les différences de position du corps, de parure, de mobilier, de structure de la tombe?

L'analyse des pratiques funéraires des populations actuelles ou subactuelles montre qu'il existe des variations dans le traitement des morts à l'intérieur d'un groupe et entre groupes contemporains. Elle révèle aussi une évolution des pratiques. L'âge au décès, le genre, le rôle, le statut social, les circonstances et les causes de la mort, sont des paramètres classiques.

Dans un échantillon aussi dispersé dans le temps et dans l'espace que l'échantillon gravettien, variation individuelle, diversification régionale et évolution diachronique se mêlent. Le faible effectif disponible, la résolution insuffisante des méthodes de datation et notre méconnaissance de certains des paramètres évoqués ( $p$. ex. le nombre d'individus de sexe inconnu ou incertain) gênent le décryptage des variations. Les deux sujets de Paglicci constituent un exemple des difficultés d'interprétation. L'un est une femme adulte, l'autre un adolescent de sexe indéterminé. Ils proviennent de deux niveaux gravettiens superposés. Les différences dans la position du corps, la parure... sont-elles liées à l'âge, au sexe, ou reflètentelles une évolution chronologique des pratiques funéraires et/ou la fréquentation du site par un groupe sans lien avec le précédent?

En restant conscient des limites inhérentes à l'échantillon et des difficultés qu'elles génèrent, il est néanmoins possible de dégager quelques faits et pistes de réflexion.

En premier lieu, les données suggèrent une diversification régionale :

- nous avons vu que certains sites (en particulier les sites italiens) se singularisent dans la manière de traiter les enfants les plus jeunes et que la proportion d'immatures varie selon les sites et les régions ;

- de même, dans les sites italiens de Ligurie la position la plus fréquente est le décubitus dorsal.

Ailleurs, les positions sont plus variées.

- en Europe centrale et orientale, les canines de renard (polaire ?) percées et l'ivoire transformé en bijoux ou en ornements du vêtement jouent un rôle majeur. Les coquillages proviennent en grande partie de gîtes fossiles. Les 600 dentales (Dentalium badense) ornant Brno 2 sont des coquillages fossiles du Miocène (Oliva 2000). En
Europe occidentale, les canines de cervidés et les coquillages marins collectés sur les plages dominent, même si l'ivoire n'est pas absent comme à Pataud en Dordogne ;

- le mobilier détermine la même partition géographique que la parure. En Europe centrale et orientale, armes, outils et objets d'art associés aux défunts sont majoritairement en ivoire de mammouth. En Europe occidentale, les matières premières dominantes sont le silex (grandes lames en Ligurie...), l'os ou le bois de cervidé (bâtons perforés d'Arene Candide 1...). Les objets d'art mobilier sont rares. Paviland, la plus au nord des sépultures d'Europe occidentale, constituerait une exception intéressante si l'association entre le corps et les objets d'ivoire décrits « à proximité » pouvait être confirmée ;

- les scapulas de mammouth du dispositif funéraire en Europe centrale et orientale illustrent aussi ce particularisme régional. Elles sont inconnues en Europe occidentale. À noter que l'Italie, qui apparaît singulière par l'absence des jeunes enfants, est pour la parure, le mobilier et l'architecture des tombes, tournée sans ambiguiité vers l'extrême ouest de l'Europe ;

- la France compte un taux élevé (60 \%) de dépôts multiples et/ou collectifs qui reste à expliquer.

3- En revanche, il est très difficile d'établir un rapport entre l'âge ou le sexe du défunt et la position du corps. Les dépôts doubles ou triples d'Italie fournissent peut-être quelques indices. L'adulte de Barma Grande, (BG2), est sur le dos tandis que les deux immatures (BG3, BG4) sont placés sur le côté gauche. À la grotte des Enfants, l'adulte (GE5) est en partie sur le ventre contrairement à l'adolescent (GE6). Age ou sexe sont deux des critères qui dans ces exemples, pourraient expliquer les différences. Cependant I'hypothèse de Formicola (1988) selon laquelle la distinction entre les sexes serait manifeste en Italie à la fois parce que les femmes sont peu représentées et parce qu'elles ne sont jamais inhumées seules, est invalidée par le pourcentage d'individus non " sexés » et parce qu'au moins trois femmes (Ostuni 1, Paglicci 25 et Barma de Caviglione 1) ont été inhumées seules.

4- De même, à l'intérieur de chacun de ces territoires, il n'y a pas de rapport clair entre âge et sexe et le dispositif funéraire, la parure ou le mobilier, qu'il s'agisse de leur nature ou de leur abondance. Lorsque la détermination du sexe et celle de l'âge au décès sont fiables, des femmes comme des hommes, des très jeunes enfants comme des adolescent et des adultes de tout âge ont été inhumés avec de la parure, du mobilier, de l'ocre dans des espaces comparables en première analyse.

5- Une diversification régionale transparaît mais le petit nombre de sépultures ne permet pas de reconnaître une évolution nette et significative des pratiques au cours du Gravettien. On remarque néanmoins qu'en Europe centrale et orientale, la parure et le mobilier des sépultures datées entre 26 et 25000 BP est pauvre. Au contraire, la parure et le mobilier qui accompagnent Brno 2, Soungir 1 à 3 , Malta 1 , plus récentes sont très spectaculaires et particulièrement abondants. 
À l'échelle de l'Europe, l'image qui se dessine est celle de groupes partageant une même logique de gestion des morts (ou de certains morts), mais qui l'expriment selon des modalités propres adaptées pour les matières premières du mobilier, de la parure et du dispositif funéraire aux stratégies d'exploitation du milieu naturel et au mode de vie. En Europe centrale et orientale, les sépultures proviennent de grands sites de plein air occupés par des groupes pour lesquels l'exploitation du mammouth joue un grand rôle (Svoboda 2003). La richesse et l'extension de ces sites évoquent des formes de " sédentarité " ou au moins des occupations répétées et longues par des groupes importants. En Europe occidentale, les sépultures proviennent de grottes et d'abris fréquentés par des groupes apparemment plus réduits et peut-être plus mobiles, orientés vers une exploitation des ressources animales différentes.

Toutefois si la spécificité régionale de la parure et du mobilier découle de choix économiques en partie contraints par l'environnement, elle montre aussi que ces éléments étaient chargés d'un poids symbolique suffisamment fort pour que les échanges/relations entre groupes dont témoignent par exemple les statuettes (Mussi 2004), ne les concernent pas.

Mais peut-on aller au-delà d'une approche descriptive de la documentation?

La pérennité et la récurrence de gestes funéraires pendant plusieurs millénaires sur de vastes territoires suggèrent des pratiques codifiées ayant un sens fort, même s'il a varié. L'existence de règles qui percent à travers les gestes funéraires identifiés implique une maîtrise forte de la société sur la gestion de la mort et sans doute une conscience de la mort, intuition qui ne peut surprendre si l'on considère les sociétés gravettiennes d'Europe telles qu'elles apparaissent à partir de l'ensemble des données archéologiques. L'étude des pratiques funéraires des populations subactuelles et actuelles montre que les conduites funéraires, bien que très variées, obéissent à des constantes universelles et que leur finalité est double. Elles s'ordonnent certes autour du mort, mais elles s'adressent aux vivants : "Socialement réglé, le rituel funéraire, répond aux besoins de l'inconscient, prolongeant au plan de l'action, donc à travers les corps, les mécanismes de défense que l'imaginaire met en place pour composer avec la peur de la mort (Thomas 1988 - p. 88). II est donc très vraisemblable que les pratiques funéraires gravettiennes répondaient à des objectifs très proches.

Se profilent aussi derrière les documents archéologiques une diversité et une complexité des rituels et de leur sens n'ayant sans doute rien à envier à celles des populations actuelles. Aussi, l'interprétation des gestes identifiés devient hasardeuse. Ainsi dans les populations actuelles, l'offrande au mort ou à une entité supérieure, l'abandon d'objets jugés « impurs » parce que mis au contact du mort et/ou lui ayant appartenu, représentent trois fonc- tions, parmi d'autres, du mobilier funéraire! II est injustifié dans le cas du Gravettien de privilégier une signification. Des études comparatives des techniques de façonnage, des stigmates d'utilisation des éléments de parure, des outils et des objets d'art constituant le mobilier, seraient nécessaires pour tenter de comprendre le sens des objets associés aux défunts. En particulier, ce type d'études pourrait permettre de déterminer si le mobilier et la parure étaient strictement conçus pour l'évènement ou si, au contraire, ils faisaient partie de la panoplie quotidienne.

En l'état actuel des données, il faut avouer que la symbolique de la parure et du mobilier, marqueurs possibles du genre, de l'âge, du rôle et du statut social, nous échappe.

Parmi les nombreuses interrogations en débat, l'existence de hiérarchies sociales en est une. Nous avons vu que le petit nombre de sépultures ne constituait pas un argument très solide en faveur d'une telle organisation.

II indique une sélection mais les critères de cette sélection ne sont pas identifiés (excepté l'âge au décès pour certains sites).

D'autres preuves, issues de l'étude des sépultures, ont été avancées. La plus classique fait référence à la richesse du mobilier et de la parure. Les sépultures les plus riches (Soungir 1-3, Brno 2, Malt'a, Arene Candide 1) marqueraient l'appartenance des défunts à un groupe social privilégié ? Cette approche implique un jugement à l'aune des critères actuels de valeur. En second lieu, l'importance relative du mobilier "constitue une base très fragile pour argumenter de la différenciation sociale au Paléolithique», le dépôt de mobilier autour des défunts pouvant relever de multiples causes (Testart 2004 - p. 184). En outre, l'abondance et la « qualité » du mobilier et de la parure des sépultures les plus dotées d'Europe centrale et orientale pourraient tout aussi bien marquer une évolution chronologique des pratiques dans cette région, puisque les sépultures les plus pauvres sont aussi les plus anciennes. En Italie, l'écart de « richesse ", en réalité peu marqué, pourrait renvoyer à une diversification régionale, des centaines de $\mathrm{km}$ séparant les sépultures de Ligurie de celles du sud de l'Italie.

En fait, le traitement des défunts, tel qu'il nous est possible de le restituer est très comparable.

Testart (2004) propose une approche de la hiérarchisation des sociétés paléolithiques fondée sur les caractéristiques des tombes multiples ${ }^{12}$. Dans diverses sociétés historiques, une partie des sépultures qui regroupent dans un espace délimité plusieurs défunts morts en même temps, reflèteraient une pratique : l'accompagnement, qui fait qu'à la mort d'un personnage important, des hommes et des femmes proches du défunt sont mis à mort et inhumés en même temps que lui. Dans cette pratique, l'asymétrie spatiale (disposition des corps et architecture de la sépulture), l'asymétrie de posture opposant le défunt principal et le ou les accompagnants, les variations de nature et

(12) Ne sont pris en compte dans la discussion que les exemples dont la simultanéité des dépôts est démontrée. 
d'abondance du mobilier funéraire attaché aux défunts, exprimeraient un rapport de dépendance en relation avec une hiérarchisation de la société. Pour le Paléolithique, et notamment pour le Gravettien, le dispositif funéraire des sépultures multiples se caractériserait «par les dispositions symétriques des corps dans un espace non hiérarchisé » selon Testart (2004 - p. 183), la tombe des deux enfants de Soungir étant pour cet auteur exemplaire. Tout rapport de dépendance serait exclu, suggérant des sociétés peu différenciées. II n'y aurait donc pas d'exemple connu d'accompagnement hiérarchique au Gravettien. Une autre lecture des données archéologiques pourrait cependant conduire à une conclusion sinon opposée, en tout cas plus nuancée. Testart considère que l'asymétrie ne peut être évoquée à propos des sépultures triples. Pourtant, les sépultures triples de Dolni Vestonice et de Barma Grande ne sont pas strictement symétriques. L'agencement des corps de la sépulture triple de Dolni Vestonice et la position des membres supérieurs de Dolni Vestonice 13, font de Dolni Vestonice 15 le personnage le plus en vue. Le troisième (DV 14) est sur le ventre, contrairement aux deux autres placés sur le dos. II n' y a pas de différences marquantes dans le mobilier associé à chacun des trois individus mais le sujet central est distingué par une plage d'ocre à hauteur du bassin.

Il est aussi excessif de parler de dispositif symétrique pour la sépulture de Barma Grande : l'adulte est sur le dos tandis que les deux adolescents placés à sa droite sont sur le côté. En revanche, les différences au niveau de la parure et du mobilier sont mineures et de même amplitude que celles observées entre les sujets des tombes individuelles du même gisement. Les détails de ces deux sépultures triples conduisent à être moins péremptoire quant à l'appréciation de leur symétrie. Le cas de Soungir 2, 3 paraît en revanche simple. Pourtant, dès lors qu'il est difficile (voire impossible) d'appréhender l'espace funéraire délimité par les paléolithiques, et en raison de la faible amplitude des écarts entre les dates ${ }^{14} \mathrm{C}$ de chaque individu ( $\mathrm{S} 1$, S2, S3), on ne peut exclure que les deux sépultures de Soungir ( 1 1 et S2/3) forment un ensemble synchrone : les enfants et leur mobilier " accompagnant " l'adulte inhumé à proximité. On a là, si l'on étend l'espace funéraire à l'emprise des deux tombes, une asymétrie spatiale et une asymétrie de posture et de «mobilier» en accord alors, selon l'optique de Testart, avec un accompagnement hiérarchique. Selon cette interprétation, le paradoxe d'armes d'adulte associées à de jeunes enfants s'efface.

L'objectif de ces dernières remarques n'est pas de conclure à une hiérarchisation dans les communautés gravettiennes, hiérarchisation que les données archéologiques sur les modes de vie et les comportements des groupes ne laissent pas percevoir, mais d'insister sur le fait que l'interprétation des documents paléolithiques n'est jamais simple. En outre, rien ne permet d'affirmer que, du Gravettien aux périodes historiques, l'asymétrie des tombes multiples a conservé une même implication.
Au-delà de la question de la hiérarchisation des sociétés paléolithiques, pour laquelle l'analyse des sépultures multiple n'apporte pas de réponse définitive, la signification de celles-ci reste posée pour trois raisons :

- la première parce qu'elles représentent à l'échelle de l'Europe un pourcentage non négligeable des sépultures (16\%) ;

- la seconde parce qu'elles témoignent d'une pratique inhabituelle dans la plupart des populations. II est en effet rare de déposer plusieurs morts dans un même espace, comme l'ont souligné de nombreux auteurs et plus récemment Chambon et Leclerc (2007) ou Boulestin (2008). Mourir au même moment ne justifie pas une telle pratique ; - la troisième, parce qu'elles renvoient à plusieurs types d'hypothèses comme nous le verrons et non à un seul type (mort violente/sacrifice ou conflit) avec pour seul argument, l'invraisemblance de morts naturelles simultanées (Buzhilova 2000 - p. 448 ; Formicola 2007).

Quelles sont leurs caractéristiques ? Six tombes gravettiennes avec inhumations simultanées indiscutables, donc des décès synchrones ${ }^{13}$ sont connues : Barma Grande 2 , 3, 4; Grotte des Enfants 5, 6 ; Veneri Parabita 1, 2 ; Soungir 2, 3 ; Dolni Vestonice 13, 14, 15 ; Krems A, B.

Elles ne sont ni spécifiques d'une région ou d'un moment du Gravettien, ni d'une classe d'âge, ni d'un sexe. Hormis Veneri Parabita, elles proviennent en majorité de grands sites d'habitat (abri ou plein air) livrant aussi des sépultures individuelles et fréquentés pendant une longue période ou de façon répétée par des groupes relativement importants.

II n'y a pas d'association type, que l'on considère l'âge ou le sexe lorsqu'il est connu. II n'existe aucune donnée permettant d'assigner un rôle et une place particulière dans le groupe à chacun de ces individus. Tous, exceptés les enfants d'Autriche étaient, semble-t-il, en capacité de participer aux activités d'une communauté de chasseurs-collecteurs. Les indices univoques en faveur de possibles liens biologiques sont rares. La seule exception serait la triple sépulture de Barma Grande (Formicola 1991), où un sillon frontal présent sur les trois crânes pourrait indiquer une relation entre les trois défunts. Le lien génétique envisagé entre les enfants de Soungir (Poltoraus et al. 2000) s'appuie sur une analyse contestée de l'ADN (cf. supra). Les deux immatures de Krems sont interprétés comme des jumeaux, hypothèse plausible, mais qui ne repose que sur une évaluation de l'âge biologique, l'étude morphométrique restant à faire.

Y-a-t-il des différences entre sépultures multiples et individuelles des mêmes gisements ou de la même région?

Pour Barma Grande 2, 3, 4, Grotte des Enfants 5, 6, Veneri Parabita 1, 2, la réponse est négative. Ni le recrutement, ni la posture, ni l'architecture, ni l'abondance ou le type de mobilier et de parure ne diffèrent tant la diversité est grande en Italie.

(13) Nous ne retenons pas l'hypothèse, certes possible, mais indémontrable, de décès échelonnés dans le temps avec préservation des corps (froid, momification...) puis inhumations simultanées. 
Dolni Vestonice 13, 14, 15 se singularisent par la disposition des corps (décubitus dorsal et procubitus), l'ensemble évoquant une mise en scène, et peut-être par la présence d'une structure protectrice de bois. En revanche, parure et mobilier sont aussi pauvres que dans les sépultures individuelles des mêmes sites.

Krems A, B ont été traités quel que soit l'aspect retenu comme les défunts des sépultures individuelles des régions les plus proches (République Tchèque).

Les armes et les outils différencient Soungir 2, 3 de l'adulte (S1). Toutefois, hormis le format inhabituel d'une partie des armes, la sépulture des deux enfants n'apparaît pas aussi exceptionnelle comparée à la sépulture moins connue de l'enfant de Malt'a entouré lui aussi d'un matériel de grande qualité et d'armes insolites au regard de son jeune âge (Mongait 1959). Quant à la disposition originale des corps des deux enfants, elle pourrait être la conséquence des dimensions de la fosse imposées par la longueur des armes associées.

Le hasard des décès suffit-il à justifier ces inhumations simultanées?

En dépit des limites inhérentes à l'échantillon (nombre réduit de sépultures, incertitudes sur leur synchronisme), une étude en cours selon la méthode proposée par B. Boulestin (2008) montre que le décès simultané de deux ou trois individus dans les gisements de la colline de Pavlov et des Baoussé-Roussé est un évènement improbable et incompatible avec une mortalité naturelle. En revanche, à Krems, Soungir et Veneri Parabita, le rôle du hasard ne peut être rejeté.

Dans le premier cas, les décès seraient liés : la mort de l'un aurait entraîné celle du ou des autres (cas de l'accompagnement) ou les décès seraient dus à une même cause (sacrifice, épidémie, famine, conflit...).

Dans le second cas, les hasards de la mortalité naturelle suffiraient à expliquer l'inhumation simultanée, ce qui ne signifie pas que cette hypothèse soit vraie.

À ce stade de la réflexion, toutes les hypothèses sont possibles et l'ignorance des causes de la mort ne simplifie pas l'interprétation.

La recherche d'éventuelles anomalies démographiques plaidant en faveur d'une démographie non naturelle (cf. Sellier 1996) se heurte aux spécificités de l'échantillon (notamment effectif réduit par site) qui rendent l'interprétation des résultats (Henry-Gambier étude en cours) très problématique. Toutefois, l'âge des défunts (très jeunes adultes, adolescents, enfants entre 9-13 ans) des Baoussé-Roussé et de Dolni Vestonice ainsi que de Soungir interpelle. En effet, dans une population à faible espérance de vie (25-30 ans), le nombre des décès est très important avant cinq ans, minimal entre 10 et 15 ans. II remonte légèrement après 15 ans pour atteindre un maximum dans les classes âgées. En revanche, les deux très jeunes enfants de Krems et les deux adultes de Veneri Parabita appartiennent à des classes d'âge à "plus haut risque ". L'hypothèse de deux décès naturels à Krems se pose d'autant plus qu'un troisième immature autour du stade périnatal a été inhumé seul à proximité. Si l'étude biologique confirmait leur parenté, celle-ci et un décès naturel à proximité du terme pourrait expliquer leur inhumation simultanée sans avoir à invoquer d'autres hypothèses.

Nous avons vu (cf.supra) que les données archéologiques ne sont pas en faveur de l'accompagnement hiérarchique, tel qu'il est décrit dans les périodes historiques : l'asymétrie des sépultures triples est peu tranchée et les trois sépultures doubles sont symétriques. L'accompagnement non hiérarchique avec suicide de l'un quand l'autre meurt (cf. Testart 2004 - p.182-183) pourrait être une alternative pour GE 5-6 et surtout pour Veneri Parabita 1, 2, sépulture isolée associant une femme et un homme. Mais on voit mal quel (s) indice (s) retenir ou privilégier pour asseoir véritablement cette hypothèse?

Deux autres points doivent être mis en avant dans le cas de Barma Grande 2, 3, 4, Grotte des Enfants 5 et 6 et Veneri Parabita 1 et 2. L'un est l'absence de spécificité par comparaison avec les sépultures individuelles voisines suggérant qu'elles s'intègrent dans un même type de « rituel » que les autres. Le second est la diversité du recrutement que l'on considère l'âge et/ou le sexe qui va à l'encontre d'individus sélectionnés pour leur âge, leur sexe ou leur rôle, leur statut, leur filiation. Ceci tend à exclure des pratiques telles que l'accompagnement hiérarchique ou le sacrifice qui supposent des dispositifs singuliers et des individus particuliers. Le rejet de l'accompagnement hiérarchique ou du sacrifice conduit alors à poser l'hypothèse de décès consécutifs à une crise de mortalité. Elle est cohérente avec la présence de plusieurs individus de la classe d'âge 12-15 ans.

Les causes d'une crise de mortalité susceptibles de se solder par plusieurs décès groupés, et l'urgence aidant, par une inhumation dans un même espace sont multiples : conflit, famine, épidémie. II n'y a aucune raison de privilégier les conflits armés (cf. Testart 2004) : aucun indice direct (blessures, armatures fichées dans l'os...) n'étaye cette conclusion. En outre, la paléopathologie des squelettes ne révèle pas de séquelle convaincante de comportements violents fréquents. Un seul cas pourrait concerner Dolni Vestonice 3, inhumé seul, qui porte la marque d'un choc grave mais non mortel dont il est évidemment impossible de dire s'il relève d'une agression et encore moins d'un conflit entre groupes voisins. Les traces les plus anciennes de blessures par arme attestées par un projectile fiché dans l'os ne remontent pas au-delà de l'Épipaléolithique (11-10 000 BP), période dynamique au cours de laquelle la capacité à innover et à expérimenter, des hommes, s'exerce dans un environnement en pleine transformation et se traduit par des modifications des modes de vie, des pratiques funéraires et vraisemblablement d'organisation des groupes. (Henry-Gambier 2001). 
Pour Soungir 1 et 2 comme pour Dolni Vestonice 13, 14, 15, l'âge des inhumés est aussi cohérent avec l'hypothèse de décès dus à une crise de mortalité mais les singularités respectives (parure, mobilier et posture des corps) de ces ensembles sont troublantes. En l'état des données, ni l'accompagnement hiérarchique, ni le sacrifice ne constituent des hypothèses satisfaisantes. Ce dernier qui resurgit périodiquement dans les études, renvoie à une vision du monde hiérarchisée. Les sociétés protohistoriques et historiques (Empires incas, aztèque, chefferies, royautés de Polynésie...) pour lesquelles cette pratique a été décrite correspondent à des sociétés fortement stratifiées, organisation inconnue chez les chasseurs-collecteurs (Testart 1993). L'image des groupes gravettiens (y compris ceux de Soungir ou de Dolni Vestonice) que les données archéologiques nous permettent de construire n'est pas en faveur d'un tel type de société. Ces deux sépultures, pourraient donc relever aussi d'une crise de mortalité. Là encore, un éventuel lien avec un épisode conflictuel ne repose sur aucun indice direct et l'hypothèse d'une famine ou d'une épidémie est défendable.

\section{CONCLUSION}

Les sépultures gravettiennes constituent incontestablement une source documentaire majeure pour la compréhension des comportements et des modes de vie des populations gravettiennes. L'analyse de ces documents permet la restitution d'une infime partie des gestes funéraires et le détail des rituels funéraires comme la signification et les croyances dont témoignent ces gestes, nous échappent.

Le petit nombre de sépultures et leur dispersion spatio-temporelle limite l'interprétation des variations observées. Une analyse réellement pluridisciplinaire de chaque sépulture intégrant les données anthropologiques et culturelles pourrait réduire le champ des possibles et ouvrir d'autres voies de réflexion. En particulier, une étude du mobilier et de la parure (origine des matières premières, type, composition, façonnage, stigmates d'utilisation, rapport au corps) reste à développer dans beaucoup de sites. Une approche conjointe des objets des tombes et de ceux abandonnés dans les habitats pourrait déboucher sur une interprétation mieux étayée du rôle et du sens des premiers. De même, une meilleure connaissance du contexte archéologique de ces sépultures constituerait un préalable nécessaire à une approche du rapport entre espace funéraire et habitat. De ce point de vue, les analyses en cours sur les ensembles de Pataud ou à venir sur ceux de Cussac (Dordogne) apporteront de nouveaux éléments. En particulier, l'étude du gisement de Cussac où au moins cinq défunts répartis sur trois emplacements d'une grotte fermée après le dépôt des corps, devrait renouveler la réflexion sur la signification des dépôts multiples/collectifs gravettiens. En outre, le synchronisme possible avec un art pariétal comme à Cussac, Vilhonneur en France (HenryGambier et al. 2007) et peut-être à Paglicci en Italie, ouvre la perspective de discuter d'un rapport entre art, monde souterrain et monde des morts/pratiques funéraires (ou mortuaire).

Des conclusions, provisoires pour certaines, peuvent être avancées. En premier lieu, la sépulture primaire dans le site d'habitat (plein air, grotte, ou abri) et plus rarement dans des cavités ornées est la seule pratique bien attestée. Elle ne concerne qu'une faible partie de la population décédée. Les critères de cette sélection sont loin d'être élucidés, même si en Italie l'âge au décès joue un rôle et que des indices fort d'un traitement spécifiques des très jeunes enfants existent. Des femmes et hommes, des adolescents et des enfants ont été l'objet de ces pratiques.

L'hypothèse d'une hiérarchisation des sociétés gravettiennes, souvent avancée à partir des inégalités de richesse du mobilier ou de la fonction supposée de certains défunts, n'est pour l'instant pas démontrée. Aucune des tombes multiples ne peut être rattachée de manière probante à des pratiques telle que l'accompagnement hiérarchique ou le sacrifice. L'hypothèse de décès s'inscrivant dans le cadre de crises de mortalité dont l'origine reste à élucider constitue en revanche une alternative.

Enfin, bien qu'une diversification régionale transparaisse à travers le mobilier et la parure, la répétition observée de gestes funéraires comparables à travers l'Europe, tend à accréditer l'idée déjà avancée à partir notamment de l'art mobilier, d'une certaine unité du Gravettien. Ce dernier point, est cohérent avec ce que l'on sait de l'Europe gravettienne à partir des caractéristiques climatiques et environnementales : l'absence de véritable rupture même si une réduction des voies de passage entre Europe occidentale et orientale a favorisé des processus de régionalisation.

\section{Remerciements}

Cette recherche a été possible grâce au soutien financier du CNRS (UMR 5199 PACEA), de la région Aquitaine (Projet « transition ») et de l'ANR (Guérope).

Mes remerciements s'adressent aux Conservateurs de Musée et de bibliothèques qui m'ont permis d'avoir accès aux documents anthropologiques et d'archives dont ils avaient la responsabilité. Je remercie aussi B. Boulestin et $\mathrm{S}$. Villotte pour les discussions et les conseils.

\section{BIBLIOGRAPHIE}

ALDHOUSE - GREEN S. 2000 b - Climate, ceremony, pilgrimage and Paviland: the "Red lady" in his palaeoecological and technoetic context.In: Aldhouse - Green S. (Ed. ), Paviland Cave and the «Red Lady». A definitive report, Wasp for SCARA: England, p. 227-246.

ALEXEEVA TI. et BADER NO. 2000 - Homo sungirensis. Upper Palaeolithic man: ecological and evolutionary aspects of the investigation. Moscow : Scientific World.

AUJOULAT N., GENESTE J. M., ARCHAMBEAU C., DELLUC M., DUDAY $H$. et HENRY-GAMBIER D. 2002- La grotte ornée de Cussac, Le Buisson-de - Cadouin (Dordogne): Premières observations. Bulletin de la Société Préhistorique française, 99, 1, p. 129 - 153. 
BALL A. 2002 - Critique of age estimation using attrition as the sole indicator. Journal of Forensic Odontostomatology, 20(2), p. 38-42.

BINANT P. 1991- Les sépultures du Paléolithique. Paris, Errance (collection archéologie aujourd'hui).

BORGOGNINI TARLI S., FORNACIARI G. et PALMA DI CESNOLA A. 1980 - Restes humains des niveaux gravettiens de la grotte Paglicci (Rignano Garganico) : contexte archéologique, étude anthropologique et notes de paléopathologie. Bulletins et Mémoires de la Société d'Anthropologie de Paris, 7, p. 125-152.

BUCKLAND W. 1823 - Reliquiae Diluvianae. London, J. Murray.

BUZHILOVA AP. 2000 - Pair and old burials In Upper Palaeolithic. Selective criteria of the buried, In : Alexeeva TI, Bader NO. 2000, Homo sungirensis. Upper Palaeolithic man: ecological and evolutionary aspects of the investigation. Moscow : Scientific World, p. 441-448.

BUZHILOVA AP. et LEBEDINSKAYA G.V. 2000 Preservation of anthropological material. In : Alexeeva TI, Bader NO. 2000, Homo sungirensis. Upper Palaeolithic man: ecological and evolutionary aspects of the investigation. Moscow : Scientific World, p. 49-53.

BOULESTIN B. 2008 - Pourquoi mourir ensemble ? À propos des tombes multiples dans le Néolithique français. Bull. Soc. Préh. Fr., 105, 1, p. 103-130.

BOULESTIN B. et DUDAY H. 2005 - Ethnologie et archéologie de la mort : de l'illusion des références à l'emploi d'un vocabulaire. In : Mordant cl., Depierre G. (Eds.), Les pratiques funéraires à l'Age du Bronze en France. Actes de la table-ronde de Sens -en-Bourgogne (Yonne), CTHS Paris, p. 17-30.

BRUŽEK J. 2002 - A Method for Visual Determination of Sex, Using the Human Hip Bone. American Journal of Physical Anthropology 117, p.157 - 168.

BRUŽEK J., FRANCISCUS R.G., NOVOTN V. et TRINKAUS E. 2006 - Assessment of Sex. In: Trinkaus E., Svoboda J. (Eds.) Early Modern Human Evolution in Central Europe, Oxford University Press, p. 46-62.

BRUŽEK J., VELEMINSKA J. et VELEMINSKY 2008 Paleodemographic Indicators and Current Paleoanthropological Methods as Applied to the Predmosti Sample. In : BRUŽEK J., VELEMINSKA J. (Eds.), Early Modern Humans from Predmosti. A new reading of old documentation. Academia, Praha, 2008, p. 59-73.

CHAMBON Ph. et LECLERC J. 2007 - les tombes multiples dans le Néolithique français : aléa statistique ou pratique institutionnalisée? Bull. Soc. Préhist. Franç., 104, 2, p. 289-306.
COPPOLA D. et VACCA E. 1995 - Les sépultures Paléolithiques de la caverne de Sainte Marie d'Agnano à Ostuni (Italie), In : Nature et Culture, décembre 1993, Liège, ERAUL, 68, p.797-810.

CREMONESI G., PARENTI R. et ROMANO S. 1972 - Scheletri paleolitici della grotta delle Veneri presso Parabita (Lecce), Atti XIV Riun. Scient, Ist. It. Preist. Protost, p. 105-117.

DJINDJIAN F., KOSLOWSKI J. et OTTE M. 1999 - Le Paléolithique supérieur en Europe, Armand Colin, 474 p.

DUARTE C. 2002 - The burial taphonomy and ritual. In: Zilhao J. et Trinkaus E. (Eds.) Portrait of an artist as a Child. The Gravettian human skeleton from the Abrigo do Lagar Velho and its archeological context. Lisbonne: Instituto Portugueses de Archeologia, (Trabalhos de Arqueologia 22), p. 187-201.

DUDAY H. 2005 - L'archéothanatologie ou l'archéologie de la mort. Objets et méthodes en Paléoanthopologie, CTHS, Paris, p. 153-205.

DUDAY H., COURTAUD P., CRUBEZY E., SELLIER P. et TILLIER AM. 1990 - L'Anthropologie de terrain: Reconnaissance et interpretation des gestes funéraires. Bull. et Mém. De la Soc. d'Anthropologie de Paris ns. 2, p. 29-50.

EINWÖGERER T., FRIESINGER H., HÄNDEL M., NEUGEBAUER-MARESCH C., SIMON U. \& TESCHLER-NICOLA M. 2006- Upper infant burials. Nature, 444, p. 285.

FORMICOLA V. 1988 - The male and the female In the Upper Paleolithic burials from Grimaldi Caves. (Liguria, Italy), Bulletin du Musée d'Anthropologie Préhistorique de Monaco, 31, p. 41-48.

FORMICOLA V. 1991 - The triplex burial of Barma Grande (Grimaldi, Italy), Homo, 39, 3-4, p. 131-143.

FORMICOLA V. 2007 - From the Sunghir Children to the Romito Dwarf. Aspects of the Upper Paleolithique Funerary Landscape. Current Anthropology, 48, 3, p.446-452.

FORMICOLA V., PONTRANDOLFI A. \& SVOBODA J. 2001 The Upper Paleolithic Triple Burial of Dolní V stonice : Pathology and Funerary Behavior American Journal of Physical Anthropology. 124, p. 189-198.

FORMICOLA V. \& BUZHILOVA A. P. 2002 - Double child burial from Sunghir (Russia): Pathology and inferences for Upper Paleolithic funerary practices. American Journal of Physical Anthropology,124, p. 189-198.

FORMICOLA V., PETTITT P.B. \& DEL LUCCHESE A. 2004 A direct AMS radiocarbon date on the Barma Grande 6 Upper Paleolithic skeleton. Curr.Anthropol., 45, 114-118.

GAMBIER D. 1996 - Les pratiques funéraires au magdalénien dans les Pyrénées françaises. «Pyrénées préhistoriques : Arts et Sociétés», CTHS, p. 243-263. 
GAMBIER D. 2000 - Aurignacian children and Mortuary practice in Western Europe. Anthropologie, XXXVIII/1, p. 5-21.

GAMBIER D., VALLADAS H., TISNERAT-LABORDE N., ARNOLD A. \& BRESSON F. 2000- Datation de vestiges humains présumés du Paléolithique supérieur par la méthode du C14 en SMA. Paléo, 16, p. 1 - 11.

HENRY-GAMBIER D. 2001 - Les enfants de Grimaldi (Grotte des Enfants site des Baoussé - Roussé, Italie). Anthropologie et Palethnologie funéraire. CTHS/RMN.

HENRY-GAMBIER D. 2002 - Les fossiles de Cro-Magnon (Les-Eyzies- de-Tayac, Dordogne) : Nouvelles données sur leur position chronologique et leur attribution culturelle, Bulletins et Mémoires de la Société d'Anthropologie de Paris, 14, 1-2, p. 89-112.

HENRY-GAMBIER D. 2005 - Evolution des pratiques funéraires en Italie au Paléolithique supérieur. In D. Vialou, J. Renault-Miskovsky, Patou-Mathis (Eds.), Comportements des hommes du Paléolithique moyen et supérieur en Europe: territoire et milieu. Actes du colloque du GDR 1945 du CNRS, Paris 8-10 janvier 2003, Liège, ERAUL 111 , p. $231-241$.

HENRY-GAMBIER D., BRUŽEK J., MURAIL P. et HOUET F. 2002 - Estimation du sexe du squelette magdalénien de SaintGermain-La- Rivière (Grironde, France). Paléo, 14, p. 205-212.

HENRY-GAMBIER D., MAUREILLE B. et WHITE R. 2004 Vestiges humains des niveaux de l'Aurignacien ancien du site de Brassempouy (Landes). Bulletins et Mémoires de la Société d'Anthropologie, Paris, 16/1-2, p. 49-87.

HENRY-GAMBIER D., BRUŽEK J., SCHMITT A., HOUËT F. et MURAIL P. 2006 - Révision du sexe et de l'âge au décès des fossiles de Cro-Magnon (Dordogne, France) à partir de l'os coxal. CR Palevol, 5, p. 735-741.

HENRY-GAMBIER D., BRUŽEK J., SCHMITT A., HOUËT F. et MURAIL P. 2007 - Changement de paradigme dans la détermination du sexe et de l'âge au décès des sujets adultes à partir du squelette: Application aux fossiles du Paléolithique supérieur d'Europe. Actes du 26 ème Congrès de la Société préhistorique française, Avignon, 20-25 septembre 2004, p. 516-525.

HENRY-GAMBIER D., BEAUVAL C., AIRVAUX J., AUJOULAT N., BARATIN J.F. \& BUISSON-CATIL J. 2007 - New hominid remains associated with gravettian parietal art (Les Garennes, Vilhonneur, France). Journal of Human Evolution, 53(6), p.747-750.

HOFFMANN A. \& WEGENER D. 2002 - The rediscovery of the Combe-Capelle skull. Journal of Human Evolution, 43, 577-581.

IAKOLEVA L. \& DJINJIAN F. 2004 - Dans les pays des steppes à mammouths, les sépultures du Paléolithique supérieur. Dossiers de l'Archéologie, 291, p. 94-95.
JELINEK J. 1991 - Découvertes d'ossements de la population gravettienne de Moravie. L'Anthropologie, 95, p. 137-154.

JELINEK J. 1992 - New Upper Paleolithic burials from Dolni Vestonice. ERAUL, 56, p. 207-228.

KEY C.A., LESLIE C.A. \& MOLLESON T. 1994 Cranial suture closure and its implications for age estimation. International Journal of Osteoarchaelogy, 4 5, p. 193-207.

KLIMA B. 1987 a - A triple burial from the Upper Paleolithic of Dolni Vestonice, Czechoslovakia. Journal of Human Evolution, 16, p. 831-835.

KLIMA B. 1987 b - Das jungpaläolitische Massengrab von Dolni Vestonice. Quartar, 37/38, p. 53-62.

KUKLIK M. 1992 - Die Reflexion über den Befunden aus dem jungpalaolitischen Dreigrab In Dolni Vestonice nach der genetischen Ansicht. Acta Mus Natl Pragae, 48, p. 148-151.

LECLERC J. 1990 - La notion de sépulture. Bull. et Mém. de la Soc. d'Anthropo. de Paris, 2, 3-4, p.13-18.

LECLERC J. et TARRETE J. 1988 - Sépulture. In Leroi Gourhan A. (Ed.), Dictionnaire de la Préhistoire, Presse Universitaires de France, Paris, p. 963-964.

MAJO T. 1992 - Ontogénèse de l'os coxal et détermination sexuelle : l'importance de l'ilium, Bulletins et Mémoires de la Société d'Anthropologie de Paris, 1-2, p. 61-74.

MAJO T., TILLIER A.M. et BRUŽEK J. 1993 - Test des fonctions discriminantes de Schutkowski impliquant l'ilium pour la détermination du sexe dans des séries d'enfants de sexe et d'âge connus. Bulletins et Mémoires de la Société d'Anthropologie de Paris, n.s., 5, 1-2, p. 61-68.

MALLEGNI F. et PARENTI R. 1972-1973 - Studio antropologico di uno scheletro giovanile d'epoca gravettiana raccolto nella grotta Paglicci (Rignano Garganico), Rivista di Antropologia, LVIII, p. 317-342.

MALLEGNI F., BERTOLDI F. et MANOLIS S. 2000 Paleobiology of two Gravettian skeletons from Veneri Cave, Parabita, Puglia, Italy. Homo, 51/2-3, p.235-257.

MAY F. 1986- Les sépultures préhistoriques. Paris, CNRS

MASSET C. 2002 - A quel âge mourraient nos ancêtres ? Population et société (INED), 380, p. 1-4.

MATIEGKA J. 1934 - Homo predmostensis. Fosilni clovek z Predmosti na Morave. I Lebky, Praha.

MATIEGKA J. 1938 - Homo predmostensis. Fosilni clovek z Predmosti na Morave. II. Lebky, Praha. 
MEZZENA F. et PALMA DI CESNOLA A. 1971 - Scoperta di una sepoltura gravettiana nelle Grotta Paglicci (Rignano Garganico). Rivista di Scienze Preistoriche, 27, p. 27-50.

MEZZENA F. et PALMA DI CESNOLA A. 1989-1990 Nuova sepoltura gravettiana nella Grotta Paglicci (Promontorio del Gargano). Rivista di Scienze Preistoriche, 42, p. 3-29.

MONGAIT A. 1959 - L'archéologie en URSS. Académie des Sciences de l'URSS. Inst. D'Hist. Cult. Mat. Eds. en langues étrangères. Moscou 1959.

MOORREES C.F.A., FANNING E.A. et HUNT E.E. 1963 Formation and Resorption of Three Deciduous Teeth in Children. American Journal of Physical Anthropology, 21, p. 205-213.

MORIN E. 1970 - L'Homme et la Mort. Paris, Le Seuil.

MOVIUS H.L. Jr. 1977 - Excavation of the Abri Pataud, Les Eyzies (Dordogne): Stratigraphy, American School of Prehistoric Research, bull. 31, Peabody Museum, Harvard University, Cambridge, Massachussetts, 167 p., plans séparés.

MURAIL P., BRUŽEK J. et BRAGA J. 1999 - New Approach to Sexual Diagnosis in Past Populations. Practical Adjustments from Van Vark's Procedure. Int. J. Osteoarchaeol. 9, p. 39-53.

MURAIL P., BRUŽEK J. \& F. HOUËT 2000 - Stability of the human pelvic sexual dimorphism pattern allows probabilistic sex diagnosis among Homo sapiens sapiens, Abstracts of the $12^{\text {th }}$ Congress of the European Anthropological Association «Millennial Perspectives : Past, Present and Future «, Cambridge, England, 8-11 September 2000, p.55-56.

MURAIL P., BRUŽEK J., HOUËT F. \& CUNHA E. 2005 DSP : a probabilistic sex diagnosis tool using world wide variation of pelvic bone measurements. Bulletins et Mémoires de la Société d'Anthropologie de Paris, 17, (3-4), p. 167-176.

MUSSI M. 1986 a- Italian Palaeolithic and Mesolithic Burials. Human Evolution, 1, 6, p. 545-556.

MUSSI M. 1986 b- On the chronology of the burials found In the Grimaldi caves. Anthrop. contemp., IX, p. 95-104.

MUSSI M. 2004-. East and South of the Alps : The MUP funerary And artistic record of Italy and Moravia compared. In : Svoboda J., Sedlackova (Eds.) : The Gravettian Along the Danube. Proceeding of the Mikulov Conference, 20-21. novembre 2002, Brno, Archeoloogicky ustav 2004, 297 s. obr. Tab. Broz., p. 252-269.

MUSSI M., FRAYER D.W. \& MACCHIARELLI R. 1989 - Les vivants et les morts. Les sépultures du Paléolithique supérieur en Italie et leur interprétation, dans I. Hershkovitz (ed.)
Proceedings of the Second Symposium on Upper Palaeolithic, Mesolithic and Neolithic Populations of Europe and the Mediterranean Basin, part II, BAR International Series, 508, p. 435-458.

NESPOULET R., CHIOTTI L., HENRY-GAMBIER D., AGSOUS S., LENOBLE A., MORALA A., GUILLERMIN P. et VERCOUTERE C. (sous-presse) - L'occupation humaine de l'abri pataud (les-Eyzies-de-Tayac, Dordogne). II y a 22000 ans : Problématique et résultats préliminaires des fouilles du niveau 2. Bull. Mém. SPF.

OAKLEY K.P., CAMPBELL B.G. \& MOLLESON T.I. 1971 Catalog of fossil Hominids. Part II : Europe, British Museum (Natural History), Londres.

ONORATINI G. et DA SILVA J. 1978 - La grotte des Enfants à Grimaldi. Les foyers supérieurs. Bulletin du Musée d'Anthropologie Préhistorique de Monaco, 22, p. 31-71.

ONORATINI G. et COMBIER J. 1996 - restes d'enfant et parure de coquillages du site gravettien du Marronnier (Saint-Remeze Ardêche): témoin de l'expansion occidentale de la culture de tradition noaillienne méditerranéenne. In : Otte M. (Ed.) Nature et Culture, Colloque de Liège 1317 décembre 1993), Liège, ERAUL, 68, p. 259-271.

OLIVA M. 2000 - The Brno II Upper Paleolithic burial. In: Roebroeks W, Mussi M, Svoboda J., Fennema K, (Eds.), Hunters of the Golden Age. The Mid Upper Palaeolithic of Eurasia (30 $000-20000$ BP. Leiden: University of Leiden, p. 143- 152.

OLIVA M. 2000-2001- Les pratiques funéraires dans le Pavlovien morave : Révision critique. Préhistoire Européenne, 16-17, p. 191-214.

ORSCHIEDT J. 2002 - Datation d'un vestige humain provenant de La Rochette (Saint-Léon-sur-Vézère, Dordogne) par la méthode du C14 en spectrométrie de masse. Paléo, 14 , p. $239-240$.

OTTE M. et NOIRET P. 2004 - Evolution du Gravettien au Moyen-Danube. In Svoboda J., Sedlackova (Eds.): The Gravettian Along the Danube. Proceeding of the Mikulov Conference, 20-21. novembre 2002, Brno, Archeoloogicky ustav 2004, 297 s. obr. Tab. Broz p. 8-32.

OVCHINNIKOV I. et GOODWIN W. 2003 - Ancient human DNA from Sungir ? Journal Human Evolution, 44 , p. 389-392.

PETTITT P. B. 2000 - The Paviland Radiocarbon dating program: reconstructing the chronology of faunal communities, carnivore activity and human occupation. In: S. Aldhouse-Green (Ed.), Paviland Cave and the «Red Lady ", Bristol: West Acad. Spec, p. 63-71.

PETTITT P. B. \& BADER O. 2000 - Direct AMS radiocarbon dates for the Sungir mid Upper Palaeolithic burials. Antiquity, 74, p. 269-270. 
PETTITT P. B. \& TRINKAUS E. 2000 - Direct radiocarbon dating of the Brno 2 Gravettian human remains. Anthropologie/ Brno, 38, p. 149-150.

PETTITT, P. B., VAN DER PLICHT H., BRONK RAMSEY C., MONGE SOARES A.M. \& ZILHAO J. 2002 - The radiocarbon chronology. In: Zilhao J., Trinkaus E. (Eds.), Portrait of an artist as a Child. The Gravettian human skeleton from the Abrigo do Lagar Velho and its archeological context. Lisbonne: Instituto Portugueses de Archeologia, (Trabalhos de Arqueologia 22), p. 132- 138.

PETTITT P. B., RICHARDS M.P., MAGGI R. \& FORMICOLA V. 2003 - The Gravettian burial known as the Prince (EI Principe): new évidence for his age and diet. Antiquity, 77, p. 15-19.

POLTORAUS A.B., KULIKOV E.E. \& LEBEDEVA I.A. 2000 The molecular analysis of DNA from the remains of three individuals fronm Sunghir site. Preliminary data. In : Alexeeva TI, Bader NO. 2000, Homo sungirensis. Upper Palaeolithic man: ecological and evolutionary aspects of the investigation. Moscow : Scientific World, p. 302-314.

RIEK-SALVATORE J.\& CLARK G.A. 2001 - Grave Markers. Current Anthropology, 42, p. 449- 478.

RIVIÈRE E. 1887- De l'Antiquité de l'Homme dans les Alpes-Maritimes. Paris, 1887.

SCHEUER L. ET BLACK S. 2000 - Developmental Juvenile Osteology. Elsevier Academic Press, Bath, 587 p.

SCHMITT A. 2001 - Variabilité de la sénescence du squelette humain. Réflexion sur les indicateurs de l'âge au décès : à la recherche d'outil performant.Thèse Université Bordeaux I, 2001, 347 p.

SCHMITT A. 2002 - Estimation de l'âge au décès des adultes : des raisons d'espérer. Bulletins et Mémoires de la Société d'Anthropologie de Paris, 14, p. 51-73.

SERGI S., PARENTI R. \& PAOLI G. 1974 - II giovane paleolitico della Caverna delle Arene Candide, Mem. Ist. Ital. Paleont. Umana, II, p. 13-38.

SHIPMAN P., FOSTER G. \& SCHOENINGER M. 1984 Burnt bones and teeth : an experimental study of color, morphology, crystal structure and shringkaye. J. Archaeol. Sci., 11, p 307.

SINITSYN A. 2004 - Les sépultures de Kostenki : Chronologie, attribution culturelle rite funéraire. In M. Otte (Ed.),La Spiritualité, Acte du Colloque de la commission 8 de I'UISPP (Paléolithique supérieur ) Liège, 10-12 décembre 2003, Liège ERAUL, 106, p. 237-244.

SELLIER P. 1996 - La mise en évidence d'anomalies démographiques et leur interprétation : population, recrutement et pratiques funéraires du tumulus de Courtesoult, In Piningre J.F. (Ed.), Nécropoles et sociétés au premier âge du fer : le tumulus de Courtesoult (Haute-Saône), Paris, MSH, Documents d'Archéologie Française, 54, p.136-142 et $188-200$.

SLADEK V., TRINKAUS E., HILLSON S. \& HOLLIDAY T. 2000 - The people of Pavlovien. The Dolni Vestonice, study 5, Brno.

SVOBODA J. 1997 - Pavlov I, Northwest I. The Upper Paleolithic Burial and Settlement Context. Brno, Archeologicky ustav 1997, 472s., obr.

SVOBODA J. 2003 - The Gravettian of Moravia: landscape, settlement, and dwellings. In: S.A. Vasilev, O. Soffer, O., J. Koslowski (Eds.), Perceived Landscapes and Built Environments, BAR international, series 1122 , Oxford, p. 121-129.

SVOBODA J. 2008 a - The Upper Paleolithic burial area at predmosti: ritual and taphonomy. Journal of Human Evolution 54, p. 15-33.

SVOBODA J. 2008 b - The Anthropological Finds; Context and taphonomy.In Veleminska, $\mathrm{Br}$ žek (Eds).Early Modern Humans from Predmosti near Prerov, Czech Republic. Academia Praha, p. 21- 34.

SVOBODA J.A., VANDERPLICHT, KUZELKA V. 2002 Upper Palaeolithic and Mesolithic human fossils from Moravia and Bohemia (Czech Republic) : some new C14 dates. Antiquity, 78, p. 957-962.

SVOBODA J. \& SEDLACKOVA 2002 - The Gravettian Along the Danube. Proceeding of the Mikulov Conference, 20-21. novembre 2002, Brno, Archeoloogicky ustav 2004, 297 s. obr. Tab. Broz.

TESTART A. 1985 - Le communisme primitif : économie et idéologie, Paris, Maison des Sciences de l'Homme.

TESTART A. 1993 - Des Dons et des Dieux, Paris, Armand Colin.

TESTART A. 2004 - Les morts d'accompagnement. La servitude volontaire. I, Errance.

THILLAUD P.L. 1985 - L'homme de Cro-Magnon et ses maladies. Dossier Histoire et Archéologie, 97, p. 67-73.

THOMAS L.V. 1980 - Anthropologie de la mort. Paris, Payot.

THOMAS L.V. 1988 - La mort. Que sais je, PUF.

TILLIER A.M. 1990 - Une controverse dépassée : L'existence de pratiques funéraires au paléolithique moyen. Les Nouvelles de l'Archéologie, 40, p. 22-24.

TILLIER A.M. 1995 - Paléoanthropologie et pratiques funéraires au Levant Méditerranéen durant le Paléolithique moyen : le cas des sujets non adultes. Paléorient, 21, 2, Anthropologie du Proche-Orient, Données récentes, p.63-76. 
TRINKAUS E. \& JELINEK J. 1997 - Human remains from the Moravian Gravettian: The Dolni Vestonice 3 postcrania. Journal of Human Evolution, 33, p. 33-82.

TRINKAUS E., SVOBODA J., WEST D.L., SLADEK V., HILLSON S.W., DROZDOVA E. \& FISAKOVA M. 2000 Human remains from the Moravian Gravettian: Morphology and taphonomy of Isolated Elements from the Dolni Vestonice II Site. Journal of Archaeological Science, 27, p. 1115-1132.

TRINKAUS E., FORMICOLA V., SVOBODA J., HILLSON S. \& HOLLIDAY T. 2001 - Dolni Vestonice 15 : pathology and persistence In the Pavlovian. J. Archaeol Sci, 28, p. 1201-1308.

TRINKAUS E. \& SVOBODA J.(Eds) 2006 - Early Modern Human Evolution in Central Europe. the people of Dolni Vestonice and Pavlov. The Dolni Vestonice Studies, vol. 12. Oxford University Press.

UBELAKER 1978 - Human skeletal Remains Excavation analysis interpretation. Washington, Taraxacum, 1978.

ULLRICH H. 1997 - Predmosti- an alternative model interpreting burial rites. Anthropologie, 34, p. 299-396.

VACCA E. \& COPPOLA D. 1993 - The Upper Paleolithic burial at the cave of Santa Maria di Agnano (Ostuni, Brindisi) : preliminary report. Rivista di Anthropologia (Roma), 71, p. 275-284.

VALLOIS H. V. \& BILLY G. 1965 - Nouvelles recherches sur les hommes fossiles de l'abri de Cro - Magnon, L'Anthropologie (Paris), 69, 1, 2, : 47 - 74.

VANHAREN M. 2002 - Les fonction de la parure au paléolithique supérieur: de l'individu à l'unité culturelle. Talence, IPGQ, (Thèse de doctorat, Université de Bordeaux 1).

VANHAREN M. \& D'ERRICO F. 2001 - La parure de l'enfant de la Madeleine (fouille Peyrony). Un nouveau regard sur l'enfance au Paléolithique supérieur. Paléo, 13, p. 201237.

VANHAREN M. \& D'ERRICO F. 2003 - The Body ornaments associated with the burial. In: Zilhao J. et Trinkaus E. (Eds.), Portrait of un artist as a Child. The Gravettian human skeleton from the Abrigo do Lagar Velho and its archéological context. Lisbonne: Instituto Portugueses de Archeologia, p. 154- 186 (Trabalhos de Arqueologia 22).
VANHAREN M. \& D'ERRICO F. 2003 - Le mobilier funéraire de la Dame de Saint-Germain-La-Rivière (Gironde) et l'origine Paléolithique des inégalités. Paléo, 15, p. 195-238.

VELEMINSKA J., BRUŽEK J. \& VELEMINSKY P. 2008 Paleodemographic Indicators and Current paleoanthropological Methods as applied.In Veleminska, $\mathrm{Br}$ žek (Eds).Early Modern Humans from Predmosti near Prerov, Czech Republic. Academia Praha, p. 59-74.

VERNEAU R. 1906 - Les grottes de Grimaldi (BaousséRoussé), Anthropologie, Monaco, II, 1.

VILLOTTE S., HENRY-GAMBIER D., MURAIL P. \& BRUŽEK J. 2007- Population Specific Tools for Sex Diagnosis in Upper Palaeolithic and Mesolithic Human fossils from Europe. Abstract (1 $\mathrm{p}$.), Paleoanthropology $A R$. Philadelphie USA (www. Paleoanthrop.org).

VILLOTTE S. 2008 - Enthésopathies et activités des hommes préhistoriques : Recherche méthodologique et application aux fossiles européens du Paléolithique supérieur et du Mésolithique.Thèse Université Bordeaux 1, France.

YOUNG T. 2000 - The Paviland ochres : characterisation and sourcing. In: Aldhouse - Green S. (Ed. ), Paviland Cave and the «Red Lady». A definitive report, Wasp for SCARA: England, p. 205-225.

ZILHAO J. 2005 - Burial Evidence for the social differenciation of Age classes in the early Upper Paleolithic. In : D. Vialou, J. Renault-Miskovsky, Patou-Mathis (Eds.), Comportements des hommes du Paléolithique moyen et supérieur en Europe: territoire et milieu. Actes du colloque du GDR 1945 du CNRS, Paris 8-10 janvier 2003, Liège, ERAUL 111, p. 231-241.

ZILHAO J. \& TRINKAUS E. 2002 - Portrait of an artist as a Child. The Gravettian human skeleton from the Abrigo do Lagar Velho and its archeological context. Lisbonne: Instituto Portugueses de Archeologia, (Trabalhos de Arqueologia 22). 


\section{Tableaux 1 à 22}




Grande-Bretagne
\begin{tabular}{|l|l|l|}
\hline Paviland & Pays -de-Galle & Grotte \\
\hline
\end{tabular}

France
\begin{tabular}{|l|l|l|}
\hline Cro-Magnon & Dordogne & Abri \\
\hline Cussac & Dordogne & Grotte \\
\hline Pataud & Dordogne & Abri \\
\hline Le Figuier & Ardêche & Grotte \\
\hline Combe-Capelle & Dordogne & Abri \\
\hline La Rochette & Dordogne & Grotte \\
\hline Les Marronniers & Ardêche & Grotte \\
\hline Portugual & & \\
\hline Lagar Velho & Vallée Lapedo & Abri \\
\hline
\end{tabular}

Italie
\begin{tabular}{|l|l|l|}
\hline Arene Candide & Ligurie & Grotte \\
\hline Barma Grande & Ligurie & Grotte \\
\hline Barma de Caviglione & Ligurie & Grotte \\
\hline Baousso da Torre & Ligurie & Grotte \\
\hline Grotte des Enfants & Ligurie & Grotte \\
\hline Paglicci & Pouilles & Grotte \\
\hline Veneri Parabita & Pouilles & Grotte \\
\hline Ostuni & Pouilles & Grotte \\
\hline
\end{tabular}

Autriche
\begin{tabular}{|l|l|l|}
\hline Krems-Wachtberg & Basse-Autriche & Plein Air \\
\hline
\end{tabular}

R. Tchèque
\begin{tabular}{|l|l|l|}
\hline Pavlov I & Moravie & Plein Air \\
\hline Dolni Vestonice I & Moravie & Plein Air \\
\hline Dolni Vestonice II & Moravie & Plein Air \\
\hline Brno (rue F. Joseph & Moravie & Plein Air \\
\hline Brno (Zabovresky) & Moravie & Plein Air \\
\hline Brno (Cerveny Kobe) & Moravie & Plein Air \\
\hline Predmost & Moravie & Plein Air \\
\hline
\end{tabular}

Russie
\begin{tabular}{|l|l|l|}
\hline Sungir & Plaine russe & Plein Air \\
\hline Kostenki & Voronezh & Plein Air \\
\hline Malt'a & Sibérie & Plein Air \\
\hline
\end{tabular}

Tableau 1 - Inventaire des sites européens ayant livré des vestiges humains en contexte sépulcral possible et attribués au Gravettien ou à des cultures contemporaines.

Table1 - European Gravettian sites with Human Remains. 


\begin{tabular}{|l|r|l|l|l|l|l|}
\hline Individus & Date Déc. & Inventeur & Culture & Datation C14 & Laboratoire \\
\hline Paviland 1 & 1823 & Buckland & Grav. anc. & $26350+-550 / 25840+-240 *$ & OxA 1815/ OxA 8025 & Pettit (2000) \\
\hline Cro-Magnon 1 à 5 & 1868 & Ouvriers & Grav. anc. & $27680+-270$ & Beta 157439 \\
\hline Cussac 1 à 5 & 2000 & Delluc, Massoulier & pas de matériel assoc. & $25120+-120^{*}$ & Henry-Gambier (2002) \\
\hline Pataud 1 à 6 & $1958-1963$ & Movius & Grav. Final & $22000+-600$ à 21 980+- 250 & OxA 162/GrN 1876 & Movius (1977) \\
\hline La Rochette 1/2 & 1910 & Hauser & Grav. & $23630+-130 *$ & OxA 11053 & Orschiedt (2002) \\
\hline Le Figuier 1 & 1947 & Huchard & Grav.? & & Onoratini et al, (1996) \\
\hline $\begin{array}{l}\text { Les Marronniers } \\
\text { (B15 à 17) }\end{array}$ & 1969 & Combier & Grav. à burin de Noailles & & Onoratini et al, (1996) \\
\hline Combe -Capelle 1 & 1909 & Hauser & ? & & & \\
\hline Lagar Velho 1 & 1998 & Mauricio, Souto & pas de matériel assoc. & $24360+-220 / 24860+-200$ & 0xA 8422/GrA 13 310 & Pettitt et al. (2002) \\
\hline
\end{tabular}

Tableau 2 - Europe occidentale (France, Grande-Bretagne, Portugal): Attributions chronologique et culturelle des vestiges humains ( ${ }^{*}$ échantillon os humain).

Table 2 - Western Europe (France, GB, Portugal): cultural context and absolute dates of the human remains (* Direct determination of the age of the human remains itself).

\begin{tabular}{|c|c|c|c|c|c|c|}
\hline Individus & Déc. & Inventeur & Culture & Datation $\mathrm{Cl} 4$ & Labo. & Référence \\
\hline Arene Candide I & 1942 & Brea, Cardini & Grav/Epigrav. Anc. & $23440+-190^{*}$ & 0xA 10700 & Pettitt et al. (2003) \\
\hline Barma Grande 1 & 1884 & Bonfils & Grav.? & en cours * & & Mussi (1986) \\
\hline Barma Grande 2 & 1892 & Abbo & Grav.? & $14990+-80$ (pollution?) ${ }^{*}$ & Beta 63510-CAM 7641 & Formicola et al. (2004) \\
\hline Barma Grande 3 à 4 & 1892 & Abbo & Grav.? & & & Mussi (1986) \\
\hline Barma Grande 5 & 1894 & Abbo & Grav.? & & & Mussi (1986) \\
\hline Barma Grande 6 & 1894 & Abbo & Grav.? & $24800+-800^{*}$ & OxA-10093 & Formicola et al. (2004) \\
\hline Barma de Caviglione I & 1872 & Rivière & Grav.? & & & Mussi (1986) \\
\hline Baousso da Torre 1 à 3 & 1873 & Rivière & Grav? & en cours * & & Mussi (1986) \\
\hline Grotte des Enfants 4 à 6 & 1901 & \begin{tabular}{|l} 
Villeneuve \\
\end{tabular} & Grav. à Burin de Noailles & en cours * & & Onoratini et al. (1978) \\
\hline Paglicci 15 & 1971 & Mezzena, Palma di Cesnola & Grav. Pte Font Robert & $24720+-420$ & & Mezzena et al. (1971) \\
\hline Paglicci 25 & 1988 & Mezzena, Palma di Cesnola & Grav. Pte Font Robert & $23470+=370$ & & Mezzena et al. (1989-1990) \\
\hline Paglicci (A à N) & 1971 & Palma di Cesnola & Grav. Pte Font Robert & $20160+-160 / 24720+-420$ & F45 - F55 & Borgognini Tarli et al. (1980) \\
\hline Veneri Parabita 1 - 2 & 1968 & Cremonesi & Grav. & & & Cremonesi et al. (1972) \\
\hline Ostuni 1 & 1991 & Coppola & Grav. & $24410+-320^{*}$ & Gif 9247 & Coppola et al. (1995) \\
\hline Ostuni 2 & 1991 & Coppola & Grav. & & & Coppola et al. (1995) \\
\hline
\end{tabular}

Tableau 3 - Europe occidentale (Italie) : attributions chronologique et culturelle des vestiges humains ( ${ }^{*}$ échantillon os humain).

Table 3 - Western Europe (Italy): Cultural context and absolute dates of the human remains.

$\left({ }^{*}\right.$ Direct determination of the age of the human remains itself). 


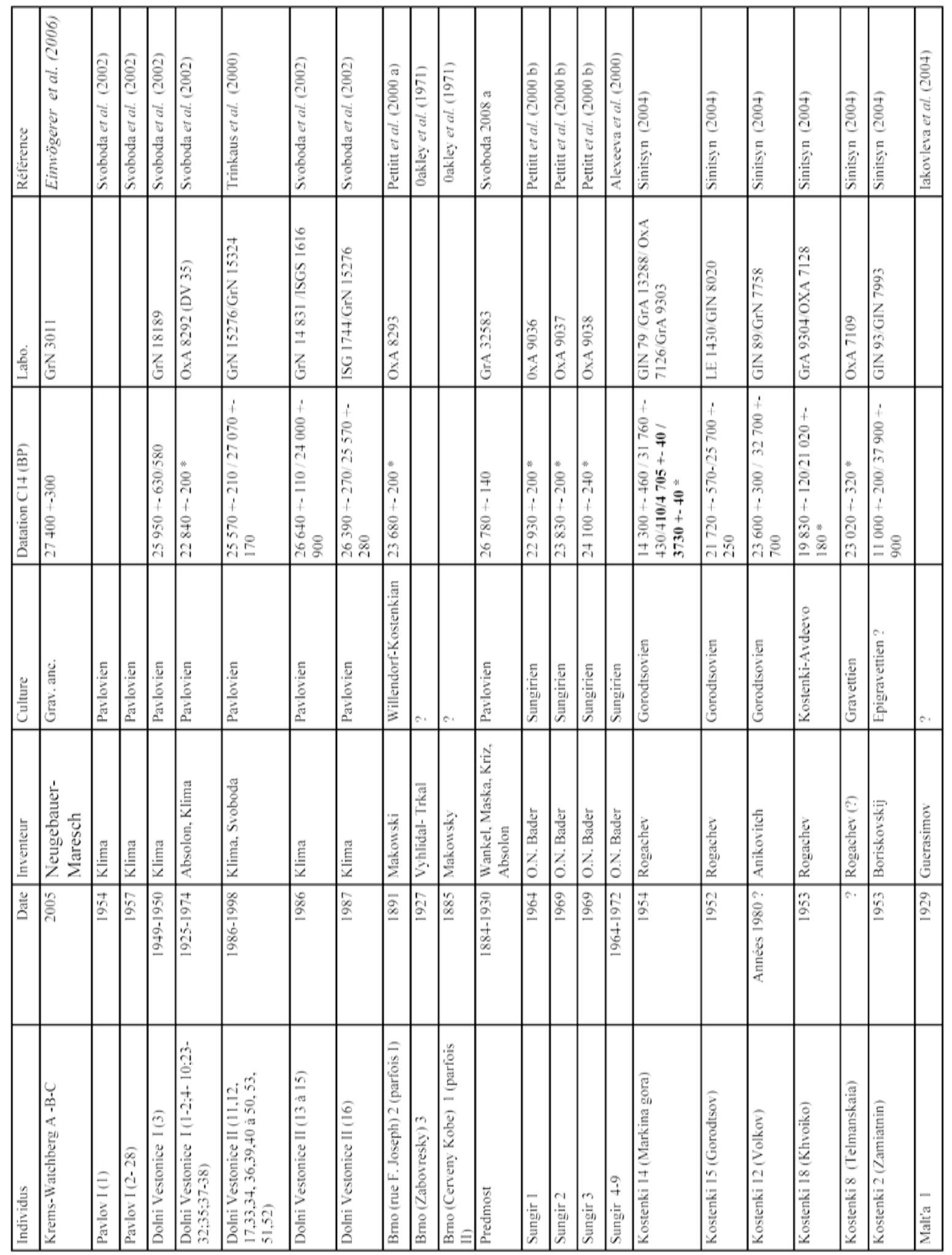

Tableau 4 - Europe centrale et orientale : attributions chronologique et culturelle des vestiges humains. ( * échantillon os humain).

Table 4 - Eastern and central Europe: Cultural context and absolute dates of the human remains. ( ${ }^{*}$ Direct determination of the age of the human remains itself). 


\begin{tabular}{|c|c|c|c|c|c|c|c|c|c|c|}
\hline & Fosse & Pierre/os & Mobilier & Ocre & Conservation & Connex. & Position & Nbre.ind.. & Lieu & Pert \\
\hline Paviland 1 & $?$ & $?$ & $?$ & oui & sq. infra-crânien incomplet (1/2 G) & oui & Déc. dorsal? & 1 & oui & oui \\
\hline Cro-Magnon 1 (4314) & $?$ & $?$ & $?$ & oui & sq. crânien/infra-crânien imcomplet & $?$ & $?$ & 1 & oui & oui \\
\hline Cro-Magnon (4316) & $?$ & $?$ & $?$ & oui & sq. crânien/infra-crânien imcomplet & $?$ & $?$ & 1 & oui & oui \\
\hline Cro-Magnon (4317) & $?$ & $?$ & $?$ & oui & sq. crânien/infra-crânien imcomplet & $?$ & $?$ & 1 & oui & oui \\
\hline Cro-Magnon (4318/4315) & $?$ & $?$ & $?$ & oui & sq. crânien/infra-crânien imcomplet & $?$ & $?$ & 1 & oui & oui \\
\hline Cro-Magnon 5 & $?$ & $?$ & $?$ & oui & sq. crânien/infra-crânien imcomplet & $?$ & $?$ & 1 & oui & oui \\
\hline Cussac locus 1 & DN & non & $?$ & $?$ & sq. infra-crânien & non & non & 1 & oui & oui \\
\hline Cussac locus 2 & DN & non & $?$ & $?$ & sq. complet & partielles & Sur le ventre & 1 & oui & oui \\
\hline Cussac locus 3 & DN & non & $?$ & $?$ & sq. infra-crânien/mandibule (s) & $?$ & $?$ & 3 & oui & oui \\
\hline Pataud (bandes V, VI, VII) 1 & $?$ & $?$ & $?$ & oui & sq. crânien/infra-crânien imcomplet & non & & 1 & oui & $?$ \\
\hline Pataud (bande VII) 2 & $?$ & $?$ & $?$ & oui & sq. infra-crânien imcomplet & non & & 1 & oui & $?$ \\
\hline Pataud (bande II) 4 & non & non & $?$ & non & sq. crânien/infra-crânien imcomplet & non & & 1 & oui & $?$ \\
\hline Pataud (bande II) 3 & non & non & $?$ & non & sq. infra-crânien imcomplet & partielles & Déc. latéral G & 1 & oui & $?$ \\
\hline Pataud (bande II) 5 & non & non & $?$ & non & sq. infra-crânien incomplet & non & & 1 & oui & $?$ \\
\hline Pataud (bandes II, III,IV) 6 & non & non & $?$ & non & sq. crânien incomplet & non & & 1 & oui & $?$ \\
\hline La Rochette 1-2 & $?$ & $?$ & $?$ & $?$ & sq. infra-crânien incomplet & $?$ & $?$ & $2 ?$ & oui & $?$ \\
\hline Le Figuier 1 & DN & $?$ & oui? & oui & sq. crânien/infra-crânien incomplet & $?$ & $?$ & 1 & oui & $?$ \\
\hline Les Marronniers (B15 à 17) & oui & non & non & oui & dent isolée, frg crâne & non & $?$ & 1 & oui & $?$ \\
\hline Lagar Velho & oui & oui? & oui? & oui & sq. crânien/infra-crânien imcomplet & oui & Déc.dorsal, mbres en ext. & 1 & non & oui \\
\hline
\end{tabular}

Tableau 5 - Europe occidentale : critère de détermination de l'intentionnalité du dépôt. DN : dépression naturelle ; Déc. : Decubitus ; sq. : squelette, Rem : Remaniement ; ? : donnée incertaine ou inconnue.

Table 5 - Western Europe: Criterion to determine the intentonality of a deposit - DN : natural grave; Déc. :Decubitus; sq. : skeleton, Pert. : disruption; ? : data uncertain or unknown. 


\begin{tabular}{|c|c|c|c|c|c|c|c|c|c|c|}
\hline & Fosse & Pierre/os & Mobilier & Ocre & Conservation & Connex. & Position & Nbre Ind. & Lieu & Pert. \\
\hline Arene Candide I & oui & oui & oui & oui & sq.complet & oui & $\begin{array}{l}\text { Déc. dorsal, mbres sup. d. fléchis, } g \text {. } \\
\text { étendu, mbres inf. étendus }\end{array}$ & 1 & oui & non \\
\hline Barma Grande 1 & $?$ & oui & oui & oui & sq. crânien/rares os infra-crâniens & oui & Déc. dorsal, mbres étendus? & 1 & oui & oui \\
\hline Barma Grande 2 & oui & non & oui & oui & sq. crânien/infra-crânien incomplet & oui & Déc. dorsal, mbres étendus & 1 & oui & $?$ \\
\hline Barma Grande 3 & oui & non & oui & oui & sq. crânien/infra-crânien incomplet & oui & Déc. latéral g. mbres sup. fléchis & 1 & oui & $?$ \\
\hline Barma Grande 4 & oui & non & oui & oui & sq. crânien/infra-crânien incomplet & oui & Déc. latéral g. mbres sup. fléchis & 1 & oui & $?$ \\
\hline Barma Grande 5 & $?$ & oui & oui & oui & sq. crânien/infra-crânien incomplet & oui & $\begin{array}{l}\text { Déc. dorsal, mbres sup. fléchis, mbres } \\
\text { inf. étendu, jambes croisées }\end{array}$ & 1 & oui & $?$ \\
\hline Barma Grande 6 & $?$ & $?$ & $?$ & $?$ & sq. crânien/infra-crânien incomplet & oui & Mbres inf. fléchis & 1 & oui & oui \\
\hline Barmade Caviglione 1 & non? & oui? & oui? & oui & sq. complet & oui & Déc. latéral g. mbres fléchis & 1 & oui & non \\
\hline Baousso da Torre 1 & non? & non & non & oui & sq. crânien/infra-crânien imcomplet & oui & Déc. dorsal, mbres étendus & 1 & oui & oui \\
\hline Baousso da Torre 2 & non? & non & non & oui & sq. crânien/infra-crânien imcomplet & oui & Déc. latéral g. & 1 & oui & oui \\
\hline Baousso da Torre 3 & non? & non & non & $?$ & sq. crânien/infra-crânien imcomplet & oui & Sur le ventre & 1 & oui & oui \\
\hline Grotte des Enfants 4 & oui & $?$ & $?$ & oui & sq. complet & oui & $\begin{array}{l}\text { Déc. dorsal, mbres sup. fléchis, mbre inf. } \\
\text { étendus }\end{array}$ & 1 & oui & non \\
\hline Grotte des Enfants 5 & oui & non & oui? & oui & sq. complet & oui & sur le ventre, mbres hyperfléchis & 1 & oui & non \\
\hline Grotte des Enfants 6 & oui & oui & oui? & oui & sq. complet & oui & Déc. dorsal, mbres fléchis & 1 & oui & non \\
\hline Paglicci 15 & non & oui & oui & oui & sq. complet & oui & $\begin{array}{l}\text { Déc. dorsal, mbres sup. fléchis, mbres } \\
\text { inf. étendus }\end{array}$ & 1 & oui & non \\
\hline Paglicci 25 & oui & non & oui & oui & sq. crânien/infra-crânien incomplet & oui & $\begin{array}{l}\text { Déc. dorsal, , mbres sup. légèrement } \\
\text { fléchis, mbres inf. étendus }\end{array}$ & 1 & oui & oui \\
\hline $\begin{array}{l}\text { Paglicci A,B, } \\
C, D, F, G, H, I, J, L, M, N\end{array}$ & non & non & non & $?$ & frgs mds, dents isolées, humérus & non & & $6 ?$ & oui & $?$ \\
\hline Veneri Parabita 1 & DN & non & oui? & oui & $1 / 2$ inf. squelette infra-crânien & oui & Déc. dorsal, mbres étendus & 1 & oui & oui \\
\hline Veneri Parabita 2 & DN & non & oui? & oui & 1/2 inf. squelette infra-crânien & oui & Déc. dorsal, mbres étendus & 1 & oui & oui \\
\hline Ostuni 1 & oui & non & oui & oui & sq. complet (+ 1 PN en place) & oui & $\begin{array}{l}\text { Déc. dorsal, mbres sup. fléchis, mains sur } \\
\text { pubis, mbres. inf.fléchis sur la G }\end{array}$ & 1 & oui & non \\
\hline Ostuni 2 & oui & non & oui & oui & sq. complet & oui & $\begin{array}{l}\text { Déc. dorsal, mbres sup. fléchis, mains sur } \\
\text { pubis, mbres. inf.fléchis sur la G }\end{array}$ & 1 & oui & non \\
\hline
\end{tabular}

Tableau 6 - Europe occidentale (Italie) : critères de détermination de l'intentionnalité du dépôt. DN : dépression naturelle ; Déc. : Decubitus ; sq. : squelette, Rem : Remaniements ? : donnée incertaine ou inconnue.

Table 6 - Western Europe (Italy) : Criterion to determine the intentonality of a deposit - DN : natural pit; Déc. :Decubitus; sq.: skeleton, Pert. : disruption; ? : data uncertain or unknown. 


\begin{tabular}{|c|c|c|c|c|c|c|c|c|c|c|}
\hline & Fosse & Pierre/os & Mobilier & Ocre & Conservation & Connex. & Position & $\begin{array}{r}\begin{array}{r}\text { Nbre } \\
\text { Ind. }\end{array} \\
\end{array}$ & Lieu & Pert \\
\hline Krems-Wachtberg A & oui & oui & Non? & oui & $\begin{array}{l}\text { sq. crânien/infra-crânien } \\
\text { complet }\end{array}$ & oui & Déc lat. G & 1 & oui & non \\
\hline Krems-Wachtberg B & oui & oui & Non? & oui & $\begin{array}{l}\text { sq. crânien/infra-crânien } \\
\text { complet }\end{array}$ & oui & Déc lat. G & 1 & oui & non \\
\hline Krems-Wachtberg C & oui & non & Non? & oui & $\begin{array}{l}\text { sq. crânien/infra-crânien } \\
\text { complet }\end{array}$ & oui & Déc lat. D & 1 & oui & non \\
\hline Pavlov I (1) & $?$ & oui & non & $?$ & $\begin{array}{l}\text { sq. crânien/infra-crânien } \\
\text { incomplet }\end{array}$ & oui & Déc. lat. D, mbres? & 1 & oui & oui \\
\hline Pavolv I (2- 28) & non & non & non & non & $\begin{array}{l}\text { frgs. crâne, Md, dents } \\
\text { isolées }\end{array}$ & non & & & oui & oui \\
\hline \multirow[t]{2}{*}{ Dolni Vestonice I (3) } & \multirow[t]{2}{*}{ oui } & \multirow[t]{2}{*}{ oui } & oui & \multirow[t]{2}{*}{ oui } & \multirow[t]{2}{*}{ sq. complet } & \multirow[t]{2}{*}{ oui } & \multirow[t]{2}{*}{ Déc. lat. $\mathrm{D}$, mbres hyperfléchis } & \multirow[t]{2}{*}{1} & \multirow[t]{2}{*}{ oui } & \multirow[t]{2}{*}{ non } \\
\hline & & & ? & & & & & & & \\
\hline $\begin{array}{l}\text { Dolni Vestonice I (1-2;4- 10;23- } \\
32 ; 35 ; 37-38)\end{array}$ & non & non & non & non & $\begin{array}{l}22 \text { éléments (crâniens, } \\
\text { dents, } 1 \text { fémur) }\end{array}$ & non & & $5 ?$ & oui & oui \\
\hline Dolni Vestonice II (13) & oui & oui? & oui? & oui & $\begin{array}{l}\text { sq. crânien/infra-crânien } \\
\text { incomplet }\end{array}$ & oui & $\begin{array}{l}\text { Déc. dorsal, mbres sup obliques vers } \\
\text { bassin DV15, mbres inf. étendus }\end{array}$ & 1 & oui & non \\
\hline Dolni Vestonice II (14) & oui & oui? & oui? & oui & $\begin{array}{l}\text { sq. crânien/infra-crânien } \\
\text { incomplet }\end{array}$ & oui & $\begin{array}{l}\text { sur le ventre, mbres sup. en légère flexion, } \\
\text { mbres inf. étendus. }\end{array}$ & 1 & oui & non \\
\hline Dolni Vestonice II (15) & oui & oui? & oui? & oui & $\begin{array}{l}\text { sq. crânien/infra-crânien } \\
\text { incomplet }\end{array}$ & oui & Déc. dorsal, mbres en extention & 1 & oui & non \\
\hline $\begin{array}{l}\text { Dolni Vestonice II }(11,12, \\
17,33,34,36,39,40 \text { à } 53)\end{array}$ & non & non & non & non & $\begin{array}{l}\text { frgs. crâne, md, infra- } \\
\text { cranien, dents isolées }\end{array}$ & non & & $6 ?$ & oui & oui \\
\hline Dolni Vestonice II (16) & oui & non & non & oui & sq.crânien/infra-crânien & oui & Déc. lat. D, mbres fléchis & 1 & oui & non \\
\hline Brno 2 & ? & $?$ & oui & oui & $\begin{array}{l}\text { sq. crânien/infra-crânien } \\
\text { incomplet }\end{array}$ & $?$ & $?$ & 1 & oui & oui \\
\hline Predmost & oui & oui? & non & non & $\begin{array}{l}\text { sq. crânien/infra-crânien } \\
\text { incomplet }\end{array}$ & $?$ & $?$ & $20 ?$ & oui & oui \\
\hline Sungir 1 & oui & non & non & oui & $\begin{array}{l}\text { sq. crânien/infra-crânien } \\
\text { incomplet }\end{array}$ & oui & $\begin{array}{l}\text { Déc. dorsal, mains sur le pubis, mbres inf. } \\
\text { en extention }\end{array}$ & 1 & oui & non \\
\hline Sungir 2 & oui & non & oui & oui & $\begin{array}{l}\text { sq. crânien/infra-crânien } \\
\text { incomplet }\end{array}$ & oui & $\begin{array}{l}\text { Déc. dorsal, mains sur le pubis, mbres inf. } \\
\text { en extention }\end{array}$ & 1 & oui & non \\
\hline Sungir 3 & oui & non & oui & oui & $\begin{array}{l}\text { sq. crânien/infra-crânien } \\
\text { incomplet }\end{array}$ & oui & $\begin{array}{l}\text { Déc. dorsal , mbres sup. le long du corps, } \\
\text { mbres inf. en extension }\end{array}$ & 1 & oui & non \\
\hline Sungir $4-5-6-7-8-9$ & ? & $?$ & $?$ & $?$ & $\begin{array}{l}\text { frgs.s crâne, Md, infra- } \\
\text { crânien, }\end{array}$ & $?$ & $?$ & $6 ?$ & oui & oui \\
\hline Kostenki 14 (Markina Gora) N² & oui & non & non & oui & $\begin{array}{l}\text { sq. crânien/infra-crânien } \\
\text { incomplet }\end{array}$ & oui & Déc. lat. G, mbres hyperfléchis & 1 & oui & non \\
\hline Kostenki 15 (Gorodstov) N³ & oui & oui & oui & oui & $\begin{array}{l}\text { sq. crânien/infra-crânien } \\
\text { incomplet }\end{array}$ & oui & Assis (?) & 1 & oui & oui \\
\hline Kostenki 12 (Volkov) N³ & non & oui? & non & non & $\begin{array}{l}\text { sq. crânien/infra-crânien } \\
\text { incomplet }\end{array}$ & oui & Déc. dorsal , mbres étendus & 1 & oui & oui \\
\hline Kostenki 18 (Khvoiko) N 4 & oui & oui? & non & non & $\begin{array}{l}\text { sq. crânien/infra-crânien } \\
\text { incomplet }\end{array}$ & oui & Déc. lat. G, mbres hyperfléchis & 1 & oui & oui \\
\hline Kostenki 8 (Telmanskaia) & $?$ & non & non & non & $\begin{array}{l}134 \text { frgs. crâniens brulés } \\
\text {, frgs. sq.infra-crânien }\end{array}$ & non & & 1 & oui & $?$ \\
\hline Kostenki 2 (Zamiatnin) & oui & oui? & non & non & $\begin{array}{l}\text { sq. crânien/infra-crânien } \\
\text { très incomplet }\end{array}$ & partielles & Assis (?) & 1 & oui & oui \\
\hline Malt'a 1 & oui & oui & oui & oui & $\begin{array}{l}\text { sq. crânien/infra-crânien } \\
\text { très incomplet }\end{array}$ & oui & $\begin{array}{l}\text { Déc. lat. G, mbres sup. étendus, mbres inf. } \\
\text { fléchis }\end{array}$ & 1 & oui & $?$ \\
\hline
\end{tabular}

Tableau 7 - Europe centrale et orientale : critères de détermination de l'intentionnalité du dépôt. DN : dépression naturelle ; Déc. : Decubitus ; sq. : squelette, Rem : Remaniements; ? donnée incertaine ou inconnue.

Table 7 - Central and Eastern Europe: Criterion to determine the intentonality of a deposit - DN : natural pit; Déc.:Decubitus; sq.: skeleton, Pert.: disruption ? : data uncertain or unknown. 


\begin{tabular}{|c|c|c|c|c|c|}
\hline Individus & Age & Sexe & Individus & Age & Sexe \\
\hline Paviland 1 & $20-29(1)$ & $\mathrm{M}(2)$ & Baousso da Torre 3 & $\mathrm{ADO}(10)$ & Inédit \\
\hline Cro-Magnon 1 (4314) & $\mathrm{A}(2)$ & $\mathrm{M}(2)$ & Grotte des Enfants 4 & $A(11)$ & $\mathrm{M}(10)$ \\
\hline Cro-Magnon (4316) & $>50 \mathrm{a}(2)$ & $F(2)$ & Grotte des Enfants 5 & $>40 \mathrm{a}(11)$ & $F(11)$ \\
\hline Cro-Magnon (4317) & $A(2)$ & INDT (2) & Grotte des Enfants 6 & ca 12-15 a (11) & INDT \\
\hline Cro-Magnon (4318/4315) & $>50$ a (2) & $M(2)$ & Paglicci 15 & ca 14 a (16) & INDT \\
\hline Cro-Magnon 5 & $0-1$ a (3) & INDT & Paglicci 25 & $20-29(1)$ & $F(1)$ \\
\hline Cussac locus 2 & A (4) & Inédit & Veneri Parabita 1 & $>40$ a (1) & $M(1)$ \\
\hline Cussac locus 1 & $\mathrm{~A}(4)$ & Inédit & Veneri Parabita 2 & $20-49$ & $\mathrm{~F}(1)$ \\
\hline Cussac locus 3 & $A(4)$ & Inédit & Ostuni 1 & $20-29(1)$ & $F(1)$ \\
\hline Cussac locus 3 & $\mathrm{~A}(4)$ & Inédit & Ostuni 2 & A & Inédit \\
\hline Cussac locus 3 & A (4) & Inédit & Krems-Wachtberg A & 9-10m. L.(12) & INDT \\
\hline Pataud (bande V, VI, VII)-1 & $20-29$ a $(1,5)$ & $\mathrm{F}(1,5)$ & Krems-Wachtberg B & 9-10m. L. (12) & INDT \\
\hline Pataud (bande VII) -2 & $0-6 \mathrm{~m}(5)$ & INDT & Krems-Wachtberg C & $0-3 m(12)$ & INDT \\
\hline Pataud (bande II) 4 & $0-1$ a (5) & INDT & Pavlov I (1) & A & INDT (1) \\
\hline Pataud (bande II) 3 & A (1et 5) & $\mathrm{F}(1)$ & Dolni Vestonice I (3) & $\mathrm{A}(1)$ & $F(1)$ \\
\hline Pataud (bande II)- 5 & A (1et 5) & $\mathrm{M}(1)$ & Dolni Vestonice II (13) & $20-29$ a (1) & $M(1)$ \\
\hline Pataud (bandes II, III, IV)- 6 & $4-5$ a (5) & INDT & Dolni Vestonice II (14) & ca 17-18 a (13) & $M(13)$ \\
\hline Le Figuier 1 & $2-4 a$ & INDT & Dolni Vestonice II (15) & $20-29$ a (1) & $M(1)$ \\
\hline Lagar Velho 1 & $2-3 a(6)$ & INDT & Dolni Vestonice II (16) & $A(1)$ & $M(1)$ \\
\hline Arene Candide I & ca 15 a (7) & INDT & Brno (rue F. Joseph) 2 (ou I) & A & INDT \\
\hline Barma Grande 1 & $A(8)$ & $M ?(8)$ & Sungir 1 & $20-49$ a (1) & $M(1)$ \\
\hline Barma Grande 2 & $A(8)$ & $M ?(8)$ & Sungir 2 & ca 13 a (14) & INDT \\
\hline Barma Grande 3 & ca 12 a (8) & INDT & Sungir 3 & ca 9 a(14) & INDT \\
\hline Barma Grande 4 & ca 12 a (8) & INDT & Kostenki 14 (Markina Gora) №2 & A(jeune-?) (15) & $M ?(15)$ \\
\hline Barma Grande 5 & $A(8)$ & IND & Kostenki 15 (Gorodstov) N³ & ca 6-7 a (15) & INDT \\
\hline Barma Grande 6 & $A(8)$ & IND & Kostenki 12 (Volkov) Nº3 & PN (15) & INDT \\
\hline Barmade Caviglione 1 & $\mathrm{~A}(9)$ & $F(9)$ & Kostenki 18 (Khvoiko) №4 & ca 9-10 a (15) & INDT \\
\hline Baousso da Torre 1 & $A(10)$ & Inédit & Kostenki 2 (Zamiatnin) & $A(>50 a-?)(15)$ & $M ?(15)$ \\
\hline Baousso da Torre 2 & $20-29$ a (1) & $M(10)$ & Malt'a 1 & 3-4 a (17) & INDT \\
\hline
\end{tabular}

Tableau 8 - Europe (Predmost exclu) : Age et sexe au décès - 1 : Villotte (2008), 2 : Henry-Gambier et al. (2005), 3 : Vallois et al. (1965), 4 : Aujoulat et al. (2002), 5 : Nespoulet et al. (S-P), 6 : Zilhao, Trinkaus (eds.) (2002), 7 : Sergi et al. (1974), 8 :

Formicola (1988), 9 : Bružek (inédit), 10 : Henry-Gambier, Villotte (ét. en cours), 16 : Mallegni (1972-1973), 12 : Einwögerer et al. (2006), 13 : Bružek et al. (2006), 14 : Buzhilova et al. (2000) ,15 : in Snitsyne (2004), 17 : Mongait (1959).

Table 8 - Europe (without Predmost): Age at death and sex - 1 : Villotte (2008), 2 : Henry-Gambier et al. (2005), 3 : Vallois et al. (1965), 4 : Aujoulat et al. (2002), 5 : Nespoulet et al. (S-P), 6 : Zilhao, Trinkaus (eds.) (2002), 7 : Sergi et al. (1974), 8 :

Formicola (1988), 9 : Bružek (inédit), 10 : Henry-Gambier, Villotte (ét. en cours), 16 : Mallegni (1972-1973), 12 : Einwögerer et al. (2006), 13 : Bružek et al. (2006), 14 : Buzhilova et al. (2000) ,15 : in Snitsyne (2004), 17 : Mongait (1959). 


\begin{tabular}{|c|c|c|c|c|c|c|c|c|c|c|c|c|c|c|c|}
\hline Ind. & $\mathbf{N}^{\circ}$ & Md. & $\mathrm{Cr}$. & Dt. & Cx. & Ol. & Main & Pied & Vert. & Fouille & Age (1) & Sexe (2) & Sexe (3) & Sexe (4) & Age (5) \\
\hline $\mathrm{P}$ & 1 & * & * & & & * & * & & & Maska & A & $\mathrm{F}$ & $M$ & & $18-22$ \\
\hline $\mathrm{P}$ & 2 & * & * & & & * & & & & Maska & $6-7 a$ & & & & 6-7 a \\
\hline $\mathrm{P}$ & 3 & & * & & * & * & * & * & * & Maska & A & $\mathrm{M}$ & $M$ & M & $35-45$ \\
\hline $\mathrm{P}$ & 4 & & * & & * & * & * & & * & Maska & A & $\mathrm{F}$ & IND & M & $15-20$ \\
\hline $\mathrm{P}$ & 5 & * & * & & & * & * & & & Maska & $15-16$ a & & $\mathrm{F}$ & & $12-14 a$ \\
\hline$P$ & 6 & * & * & & & & & & & Maska & $2-3 a$ & & & & $2-4 a$ \\
\hline $\mathrm{P}$ & 7 & * & * & & & * & & & & Maska & $12-14 \mathrm{a}$ & & & & $12-14 a$ \\
\hline P & 8 & * & * & & * & * & & & & Maska & 4 ans & & & & $\mathrm{nr}$ \\
\hline $\mathrm{P}$ & 9 & & * & & * & * & * & * & * & Maska & AJ & $\mathrm{M}$ & $M$ & $\mathbf{F}$ & $\mathrm{nr}$ \\
\hline $\mathrm{P}$ & 10 & & * & & * & * & * & * & * & Maska & $\mathrm{AJ}$ & $\mathrm{F}$ & $\mathrm{F}$ & M & $\mathrm{nr}$ \\
\hline $\mathrm{P}$ & 11 & * & $?$ & * & $?$ & $?$ & $?$ & $?$ & $?$ & Maska & $6 \mathrm{~m}$ & & & & $\mathrm{nr}$ \\
\hline $\mathrm{P}$ & 12 & * & $?$ & * & $?$ & * & $?$ & $?$ & $?$ & Maska & $4 \mathrm{~m}$ & & & & $\mathrm{nr}$ \\
\hline $\mathrm{P}$ & 13 & * & $?$ & * & $?$ & $?$ & $?$ & $?$ & $?$ & Maska & $2 \mathrm{~m}$ & & & & $\mathrm{nr}$ \\
\hline $\mathrm{P}$ & 14 & * & * & & * & * & * & * & * & Maska & A vieux & $\mathrm{M}$ & & M & $\mathrm{nr}$ \\
\hline$P$ & 15 & * & & & & & & & & Maska & infant & & & & $\mathrm{nr}$ \\
\hline $\mathrm{P}$ & 16 & & * & & & & & & & Maska & infant & & & & $\mathrm{nr}$ \\
\hline$P$ & 17 & * & & & & & & & & Maska & infant & & & & $\mathrm{nr}$ \\
\hline $\mathrm{P}$ & 18 & * & * & & & & & & & Maska & AJ & $\mathrm{M}$ & $M$ & & $\mathrm{nr}$ \\
\hline$P$ & 19 & * & & & & & & & & Maska & A & & & & $\mathrm{nr}$ \\
\hline $\mathrm{P}$ & 20 & *? & & * & & & & & & Maska & $9-10 a$ & & & & $\mathrm{nr}$ \\
\hline$P$ & 21 & * & & & & & & & & Wankel & A & $\mathrm{F}$ & & & $\mathrm{nr}$ \\
\hline $\mathrm{P}$ & 22 & & * & & * & & * & & & Kriz & $9-11$ a & & $M$ & & $\mathrm{nr}$ \\
\hline P & 23 & & * & & * & & & & & Kriz & ADO & M & & & $\mathrm{nr}$ \\
\hline$P$ & 24 & * & & & & & & & & Kriz & $<10 a$ & & & & $6-10 a$ \\
\hline $\mathrm{P}$ & 25 & * & & & & & & & & $?$ & $<12 \mathrm{a}$ & & & & $6-10 a$ \\
\hline$P$ & 26 & * & & & & & & & & Maska & A & $\mathrm{F}$ & & & $\mathrm{nr}$ \\
\hline $\mathrm{P}$ & 27 & & & & * & * & * & * & & Absolon & A & & & & $\mathrm{nr}$ \\
\hline$P$ & 28 & & & & & * & & & & Kriz & A & & & & $\mathrm{nr}$ \\
\hline $\mathrm{P}$ & 29 & & & & & * & & & & Kriz & A & & & & $\mathrm{nr}$ \\
\hline $\mathrm{P}$ & 30 & * & & & & & & & & $?$ & A & & & & $\mathrm{nr}$ \\
\hline
\end{tabular}

Tableau 9 - République Tchèque (Predmost) : Age au décès et sexe (1, 2, : Matiegka (1934-1938), 3 : Veleminska in : Bruzek et al. (2008), 4 : Bružek in Bružek et al. 2008).

Table 9 - Czech Republic (Predmost) : Age at death and sex (1, 2, : Matiegka (1934-1938), 3 : Veleminska in : Bruzek et al. (2008), 4 : Bružek in Bružek et al. 2008). 


\begin{tabular}{|l|l|l|l|l|}
\hline & Conn. & $\begin{array}{l}\text { Conn. } \\
\text { Labiles }\end{array}$ & $\begin{array}{l}\text { Parure en } \\
\text { place }\end{array}$ & Petits os \\
\hline Paviland 1 & oui & $?$ & $?$ & oui \\
\hline Cro-Magnon 1 (4314) & $?$ & $?$ & $?$ & oui \\
\hline Cro-Magnon (4316) & $?$ & $?$ & $?$ & oui \\
\hline Cro-Magnon (4317) & $?$ & $?$ & $?$ & oui \\
\hline Cro-Magnon (4318/4315) & $?$ & $?$ & $?$ & oui \\
\hline Cro-Magnon 5 & $?$ & $?$ & $?$ & oui \\
\hline Cussac locus 1 & non & non & $?$ & oui \\
\hline Cussac locus 2 & oui & $?$ & $?$ & oui \\
\hline Cussac locus 3 & $?$ & $?$ & $?$ & oui \\
\hline Pataud (bandes V, VI,VII) 1 & non & non & non & oui \\
\hline Pataud (bande VII) 2 & non & non & non & oui \\
\hline Pataud (bande II) 4 & non & non & non & oui \\
\hline Pataud (bande II) 3 & oui & oui & non & oui \\
\hline Pataud (bande II) 5 & non & non & non & non \\
\hline Pataud (bandes II, II, IV) 6 & non & non & non & non \\
\hline La Rochette 1-2 & $?$ & $?$ & $?$ & non \\
\hline Le Figuier 1 & $?$ & $?$ & $?$ & oui \\
\hline Les Marronniers (B15 à 17) & non & non & non & non \\
\hline Lagar Velho 1 & oui & oui & non & oui \\
\hline
\end{tabular}

Tableau 10 - Europe occidentale : critères de détermination du type de dépôt (primaire/ secondaire). ? donnée incertaine ou inconnue).

Table 10 - Western Europe: Primary or secondary burials. ? data uncertain or unknown.

\begin{tabular}{|l|l|l|l|l|}
\hline & Conn. & Conn. Labiles & Parure en place & $\begin{array}{l}\text { Petits } \\
\text { os }\end{array}$ \\
\hline Arene Candide I & oui & oui & oui & oui \\
\hline Barma Grande 1 & oui & $?$ & $?$ & $?$ \\
\hline Barma Grande 2 & oui & $?$ & oui ? & oui \\
\hline Barma Grande 3 & oui & $?$ & oui ? & oui \\
\hline Barma Grande 4 & oui & $?$ & oui ? & oui \\
\hline Barma Grande 5 & oui & oui & $?$ & oui \\
\hline Barma Grande 6 & oui & $?$ & $?$ & $?$ \\
\hline Barma de Caviglione 1 & oui & oui & oui & oui \\
\hline Baousso da Torre 1 & oui & $?$ & $?$ & oui \\
\hline Baousso da Torre 2 & oui & oui & oui & oui \\
\hline Baousso da Torre 3 & oui & $?$ & pas de parure & oui \\
\hline Grotte des Enfants 4 & oui & oui & oui & oui \\
\hline Grotte des Enfants 5 & oui & oui & $?$ & oui \\
\hline Grotte des Enfants 6 & oui & oui & $?$ & oui \\
\hline Paglicci 15 & oui & oui & oui & oui \\
\hline Paglicci 25 & oui & oui & oui & oui \\
\hline Paglicci A,B, C,D,F,G,H,I,J,L,M,N & non & non & & \\
\hline Veneri Parabita 1 & oui & $?$ & non & oui \\
\hline Veneri Parabita 2 & oui & $?$ & non & oui \\
\hline Ostuni 1 & oui & oui & oui & oui \\
\hline Ostuni 2 & oui & oui & oui & oui \\
\hline
\end{tabular}

Tableau 11 - Europe occidentale : Position de la tête, orientation du corps et éléments architecturaux. (BCF : bloc crânio-facial ? donnée inconnue ou incertaine).

Table 11 - Western Europe: Head, body orientation and component. BCF : Skull and Mandible ? data uncertain or unknown. 


\begin{tabular}{|l|l|l|l|l|}
\hline & Conn. & Conn. Labiles & Parure en place & $\begin{array}{l}\text { Petits } \\
\text { os }\end{array}$ \\
\hline Individus & CR1A & CR1B & CR2 & CR3 \\
\hline Krems-Wachtberg A & oui & oui & oui & oui \\
\hline Krems-Wachtberg B & oui & oui & & oui \\
\hline Krems-Wachtberg C & oui & oui & & oui \\
\hline Pavlov I (1) & conn. lâches & non & non & non \\
\hline Pavolv I (2- 28) & non & non & non & non \\
\hline Dolni Vestonice I (3) & oui & oui & $?$ & oui \\
\hline Dolni Vestonice I (1-2;4- 10;23-32;35;37-38) & non & non & & rare \\
\hline Dolni Vestonice II (13-14-15) & oui & oui & oui & oui \\
\hline Dolni Vestonice II (11,12, 17,33,34,36,39,40 à 53) & non & non & non & oui \\
\hline Dolni Vestonice II (16) & oui & oui & $?$ & oui \\
\hline Brno 2 & $?$ & $?$ & $?$ & non \\
\hline Predmost & $?$ & $?$ & $?$ & oui \\
\hline Sungir 1 & oui & oui & oui & oui \\
\hline Sungir 2 & oui & oui & oui & oui \\
\hline Sungir 3 & oui & oui & oui & oui \\
\hline Sungir 4-5 & non & non & non & non \\
\hline Sungir 6-9 & $?$ & $?$ & $?$ & $?$ \\
\hline Kostenki 14 (Markina Gora) & oui & oui & non & oui \\
\hline Kostenki 15 (Gorodstov) & non & non & non & oui \\
\hline Kostenki 12 (Volkov) & oui & $?$ & non & oui \\
\hline Kostenki 18 (Khvoiko) & oui & $?$ & non & oui \\
\hline Kostenki 8 (Telmanskaia) & non & non & non & oui \\
\hline Kostenki 2 (Zamiatnin) & oui & $?$ & non & oui \\
\hline Malt'a 1 & oui & $?$ & $?$ & oui \\
\hline & & & & \\
\hline & on & \\
\hline
\end{tabular}

Tableau 12 - Europe centrale et orientale : critères de détermination du type de dépôt (primaire/secondaire). ? donnée incertaine ou inconnue.

Table 12 - Central and eastern Europe western Europe: Primary or secondary burials.

? data uncertain or unknown. 


\begin{tabular}{|c|c|c|c|}
\hline Individus & $\begin{array}{l}\text { Position observée } \\
\text { du BCR }\end{array}$ & $\begin{array}{l}\text { Orientation } \\
\text { corps }\end{array}$ & \begin{tabular}{|l}
$\begin{array}{l}\text { Eléments } \\
\text { architecturaux }\end{array}$ \\
\end{tabular} \\
\hline Paviland 1 & BCR Abs. & $\mathrm{N}-\mathrm{S}$ & $\begin{array}{l}2 \text { pierres, tête et pieds } \\
\text { (?) }\end{array}$ \\
\hline $\begin{array}{l}\text { Cro-Magnon } 1 \\
(4314)\end{array}$ & $?$ & ? & $?$ \\
\hline $\begin{array}{l}\text { Cro-Magnon } \\
(4316)\end{array}$ & $?$ & $?$ & $?$ \\
\hline $\begin{array}{l}\text { Cro-Magnon } \\
\text { (4317) }\end{array}$ & $?$ & $?$ & $?$ \\
\hline $\begin{array}{l}\text { Cro-Magnon } \\
(4318 / 4315)\end{array}$ & $?$ & $?$ & $?$ \\
\hline Cro-Magnon 5 & $?$ & ? & $?$ \\
\hline Cussac locus 2 & BCR déplacés & $?$ & non \\
\hline $\begin{array}{l}\text { Cussac locus } 1 \text { et } \\
3\end{array}$ & $?$ & $?$ & non \\
\hline $\begin{array}{l}\text { Pataud (bandes } \\
\mathrm{V}, \mathrm{VI}, \mathrm{VII}) 1\end{array}$ & $\begin{array}{l}\text { BCR isolé } \\
\text { Md./Cr. } \\
\text { connexion lâche }\end{array}$ & $?$ & $\begin{array}{l}3 \text { pierres autour du } \\
\text { crâne? }\end{array}$ \\
\hline $\begin{array}{l}\text { Pataud (bande } \\
\text { VII) } 2\end{array}$ & BCR Abs. & $?$ & non \\
\hline $\begin{array}{l}\text { Pataud (bande II) } \\
4\end{array}$ & Cr. frg. & $?$ & non \\
\hline $\begin{array}{l}\text { Pataud (bande II) } \\
3\end{array}$ & BCR Abs. & $?$ & non \\
\hline $\begin{array}{l}\text { Pataud (bande II) } \\
5\end{array}$ & BCR Abs. & $?$ & non \\
\hline $\begin{array}{l}\text { Pataud (bandes } \\
\text { II, III, IV) } 6\end{array}$ & Cr. frg. & $?$ & non \\
\hline Le Figuier 1 & Rotation vers Est & $?$ & $?$ \\
\hline Lagar Velho 1 & BCR déplacés & NE-SW & Linceul de peau (?) \\
\hline
\end{tabular}

Tableau 13 - Europe occidentale : Position de la tête, orientation du corps et éléments architecturaux. BCF : bloc crânio-facial. ? donnée inconnue ou incertaine.

Table13 - Western Europe: Head, body orientation and component. BCF : Skull and Mandible. ? : data uncertain or unknown.

Tableau 14 - Europe occidentale (Italie) : position de la tête, orientation du corps et éléments architecturaux. BCF : bloc crânio-facial. ? donnée inconnue ou incertaine.

Table 14 - Western Europe (Italy): Head, body orientation and component. BCF : Skull and mandible. ? : data uncertain or unknown.

\begin{tabular}{|c|c|c|c|}
\hline Individus & Position observée BCR & Orientation corps & Eléments architecturaux \\
\hline Arene Candide I & Rotation G & S-N & Pierres sur les pieds et les mains, fond fosse empierré \\
\hline Barma Grande 1 & $?$ & N-S & Pierres à hauteur de la tête? \\
\hline Barma Grande 2 & Rotation G (?) & $E-W$ & $?$ \\
\hline Barma Grande 3 & Rotation G & $E-W$ & $?$ \\
\hline Barma Grande 4 & Rotation G & $E-W$ & $?$ \\
\hline Barma Grande 5 & Axiale & S-N & Trois dalles au-dessus du corps \\
\hline Barma Grande 6 & $\mathrm{IM}$ & N-S? & $?$ \\
\hline Barmade Caviglione 1 & Rotation G & SE-NW & Bassin appuyé sur des pierres \\
\hline Baousso da Torre 1 & Axiale & NW-SE & non \\
\hline Baousso da Torre 2 & Axiale & NW-SE & non \\
\hline Baousso da Torre 3 & Cr.frg & NW-SE & non \\
\hline Grotte des Enfants 4 & Rotation G & $\mathrm{N}-\mathrm{S}$ & non \\
\hline Grotte des Enfants 5 & Face contre terre & $\mathrm{N}-\mathrm{S}$ & non \\
\hline Grotte des Enfants 6 & Rotation D & $\mathrm{N}-\mathrm{S}$ & Coffrage de pierres autour de la tête \\
\hline Paglicci 15 & Rotation D & SW-NE & Pierres \\
\hline Paglicci 25 & Axiale, incliné $G$ & $\mathrm{~N}-\mathrm{S}$ & non \\
\hline Veneri Parabita 1 & BCR Absent & S-N & non \\
\hline Veneri Parabita 2 & BCR Absent & S-N & non \\
\hline Ostuni 1 & Incliné G & S-N & $?$ \\
\hline Ostuni 2 & $?$ & S-N & $?$ \\
\hline
\end{tabular}




\begin{tabular}{|c|c|c|c|c|c|c|c|c|}
\hline Individus & Parure & Coquillages & \begin{tabular}{|l|} 
Vert. poissons \\
\end{tabular} & Canines renard & $\begin{array}{l}\text { Canines } \\
\text { cervidés }\end{array}$ & \begin{tabular}{|l} 
Parure ivoire/os \\
\end{tabular} & Outils/Armes/Minéraux & Faune \\
\hline Arene Candide I & Abondante & \begin{tabular}{|l|} 
oui \\
\end{tabular} & & & \begin{tabular}{|l|l} 
oui \\
\end{tabular} & pendeloques & Bâton percé/grde lame silex & Os d'écureuils \\
\hline Barma Grande 1 & $?$ & $?$ & & & & non & 3 grdes lames de silex & ? \\
\hline Barma Grande 2 & Rare & oui & oui & & oui & pendeloques & 1 grde lame de silex & oui (?) \\
\hline Barma Grande 3 & Rare & \begin{tabular}{|l|} 
oui \\
\end{tabular} & oui & & \begin{tabular}{|l|} 
oui \\
\end{tabular} & \begin{tabular}{|l} 
pendeloques \\
\end{tabular} & 1 grde lame de silex & oui (?) \\
\hline Barma Grande 4 & Rare & oui & oui & & & pendeloques & 1 grde lame de silex & oui (?) \\
\hline Barma Grande 5 & Rare & oui & & & oui & oui & Gypse & $?$ \\
\hline Barma Grande 6 & Rare & oui & & & & non & $?$ & ? \\
\hline Barma de Caviglione 1 & Abondante & oui & & & oui & non & lame de silex, poinçon os? & non \\
\hline Baousso da Torre 1 & Abondante & oui & & & & non & Lame de silex? & non \\
\hline Baousso da Torre 2 & Abondante & oui & & & oui & non & ? & non \\
\hline Baousso da Torre 3 & Absente & & & & & non & non & non \\
\hline Grotte des Enfants 4 & Rare & oui & & & oui & non & ? & ? \\
\hline Grotte des Enfants 5 & Rare & oui (?) & & & & non & Galet (?) & $?$ \\
\hline Grotte des Enfants 6 & Rare & oui & & & & non & Galet (?) & ? \\
\hline Paglicci 15 & Rare & oui & & & oui & non & Hématite/outils silex et os & non \\
\hline Paglicci 25 & Rare & oui & & & oui & non & Outils de silex & non \\
\hline Veneri Parabita 1 & Rare & & & & oui & non & ? & ? \\
\hline Veneri Parabita 2 & Rare & & & & oui & non & ? & ? \\
\hline Ostuni 1 & Abondante & oui & & & oui & non & Outils os et silex & oui? \\
\hline Ostuni 2 & Abondante & oui & & & oui & non & Outils os et silex & $?$ \\
\hline
\end{tabular}

Tableau 15 - Europe centrale et orientale : position de la tête, orientation du corps et éléments architecturaux. BCF : bloc crânio-facial. ? donnée inconnue ou incertaine.

Table 15 - Central and eastern Europe: Head, body orientaton and component. BCF : skull and Mandible. ? data uncertain or unknown.

\begin{tabular}{|c|c|c|c|c|c|c|c|c|}
\hline Individus & Parure & Coquillages & $\begin{array}{l}\text { Vert. } \\
\text { poissons }\end{array}$ & $\begin{array}{l}\text { Canines } \\
\text { renard }\end{array}$ & $\begin{array}{l}\text { Canines } \\
\text { cervidés }\end{array}$ & Parure ivoire/os & Outils/Armes/Minéraux & Faune \\
\hline Paviland 1 & Abondante & oui & non & non & non & oui? & oui (?) & $\begin{array}{l}\text { crâne } \\
\text { mammouth (?) }\end{array}$ \\
\hline $\begin{array}{l}\text { Cro-Magnon 1- } \\
5\end{array}$ & Abondante & oui & & & & $\begin{array}{l}2 \text { pendeloques } \\
\text { en ivoire? }\end{array}$ & $?$ & $?$ \\
\hline $\begin{array}{l}\text { Cussac locus } 1 \text {, } \\
\text { locus 2, locus } 3\end{array}$ & $?$ & $?$ & $?$ & $?$ & $?$ & $?$ & ? & $?$ \\
\hline Pataud 1-2 & Abondante & $?$ & non & non & $?$ & perles en ivoire & $?$ & $?$ \\
\hline $\begin{array}{l}\text { Pataud } 3,4,5 \text {, } \\
6\end{array}$ & $?$ & & & & & & $?$ & $?$ \\
\hline Le Figuier 1 & Rare & oui & non & non & non & non & oui (?) & non \\
\hline Lagar Velho 1 & Rare & oui & non & non & oui & non & non & oui (?) \\
\hline
\end{tabular}

Tableau 16 - Europe occidentale : objets associés aux vestiges humains. ? : donnée inconnue ou incertaine.

Table 16 - Western Europe: Associated objects with human remains. ? : data uncertain or unknown 


\begin{tabular}{|c|c|c|c|c|c|c|c|c|}
\hline Individus & Parure & Coquillages & Vert. poissons & Canines renard & $\begin{array}{l}\text { Canines } \\
\text { cervidés }\end{array}$ & Parure ivoire/os & Outils/Armes/Minéraux & Faune \\
\hline Arene Candide I & Abondante & oui & & & oui & pendeloques & Bâton percé/grde lame silex & Os d'écureuils \\
\hline Barma Grande 1 & $?$ & $?$ & & & & non & 3 grdes lames de silex & $?$ \\
\hline Barma Grande 2 & Rare & oui & oui & & oui & pendeloques & 1 grde lame de silex & oui (?) \\
\hline Barma Grande 3 & Rare & oui & oui & & oui & pendeloques & 1 grde lame de silex & oui (?) \\
\hline Barma Grande 4 & Rare & oui & oui & & & pendeloques & 1 grde lame de silex & oui (?) \\
\hline Barma Grande 5 & Rare & oui & & & oui & oui & Gypse & $?$ \\
\hline Barma Grande 6 & Rare & oui & & & & non & $?$ & $?$ \\
\hline Barma de Caviglione 1 & Abondante & oui & & & oui & non & lame de silex, poinçon os? & non \\
\hline Baousso da Torre 1 & Abondante & oui & & & & non & Lame de silex? & non \\
\hline Baousso da Torre 2 & Abondante & oui & & & oui & non & $?$ & non \\
\hline Baousso da Torre 3 & Absente & & & & & non & non & non \\
\hline Grotte des Enfants 4 & Rare & oui & & & oui & non & $?$ & $?$ \\
\hline Grotte des Enfants 5 & Rare & oui (?) & & & & non & Galet (?) & $?$ \\
\hline Grotte des Enfants 6 & Rare & oui & & & & non & Galet (?) & $?$ \\
\hline Paglicci 15 & Rare & oui & & & oui & non & Hématite/outils silex et os & non \\
\hline Paglicci 25 & Rare & oui & & & oui & non & Outils de silex & non \\
\hline Veneri Parabita 1 & Rare & & & & oui & non & $?$ & $?$ \\
\hline Veneri Parabita 2 & Rare & & & & oui & non & $?$ & $?$ \\
\hline Ostuni 1 & Abondante & oui & & & oui & non & Outils os et silex & oui? \\
\hline Ostuni 2 & Abondante & oui & & & oui & non & Outils os et silex & $?$ \\
\hline
\end{tabular}

Tableau 17 - Europe occidentale (Italie) : objets associés aux vestiges humains. ? : donnée inconnue ou incertaine.

Tableau 17 - Western Europe (Italy): Associated objects with human remains. ? : data uncertain or unknown. 


\begin{tabular}{|c|c|c|c|c|c|c|c|c|c|c|c|c|c|c|c|c|c|c|c|c|c|}
\hline 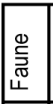 & 흘 & 듣 & 들 & 흘 & 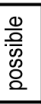 & 흐 & Бَ & Бَ & 흐 & $\alpha$ & 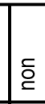 & 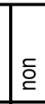 & 跔 & 흐드. & 站 & ‡ & 흐 & 흐 & 흗 & Б & ㅎ \\
\hline 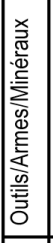 & 立 & 흐 & \begin{tabular}{|l} 
ㅎ \\
\end{tabular} & \begin{tabular}{|c} 
흐 \\
\end{tabular} & 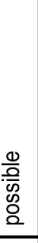 & 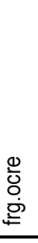 & 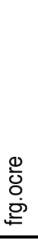 & 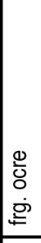 & \begin{tabular}{|l} 
ㅎ \\
\end{tabular} & '̄ & 흐 & 高 & 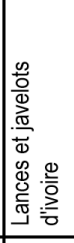 & 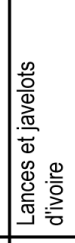 & Б亏 & 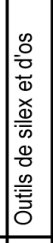 & 흐 & 흐 & 흐 & 흐 & 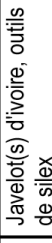 \\
\hline$\frac{0}{\frac{9}{2}}$ & & & & 들 & 站 & 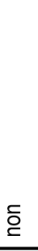 & \begin{tabular}{|l}
$\underline{\underline{c}}$ \\
\end{tabular} & 站 & 흐 & 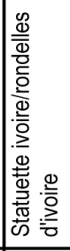 & Б흐 & 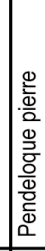 & 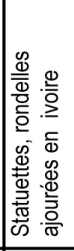 & 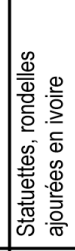 & ¿ & ᄃ & 站 & 酸 & 站 & ᄃ气 & 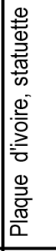 \\
\hline 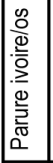 & 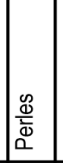 & & & 흘 & ธิ & 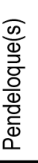 & 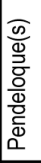 & 흗 & 흘 & |흘 & & 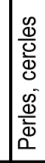 & 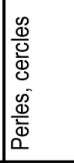 & 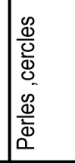 & 흘 & ฮ & 흘 & 흘 & 흘 & 흐 & 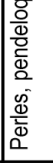 \\
\hline $\mid$ & & & & & & & & & & & & & & & & & & & & & \\
\hline 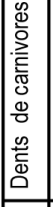 & & & & & $\frac{\mathrm{D}}{\mathrm{i}}$ & 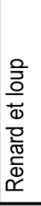 & 1 & 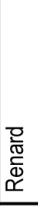 & 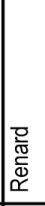 & & & 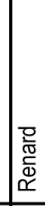 & 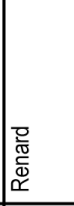 & 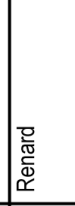 & & 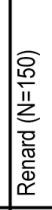 & & & & & \\
\hline 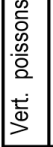 & & & & & & & & & & & & & & & & & & & & & \\
\hline 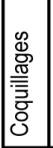 & & & & & & '亏 & & & & 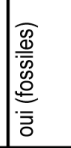 & & & & & & & & & & & 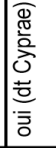 \\
\hline 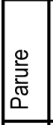 & $\overline{\bar{z}}$ & ¿ & 흘 & 흘 & 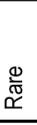 & $\frac{d}{\delta}$ & 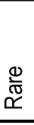 & 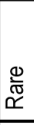 & 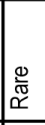 & & ธ흐 & 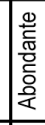 & 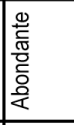 & 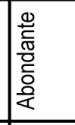 & 흗 & 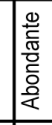 & 立 & 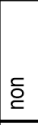 & $\overline{\underline{c}}$ & 흐 & 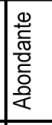 \\
\hline 产 & 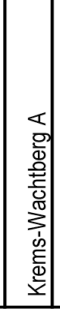 & 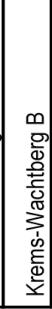 & 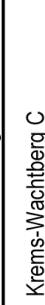 & 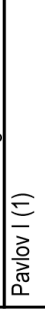 & 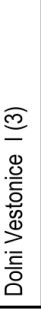 & 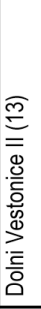 & 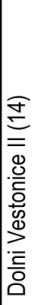 & 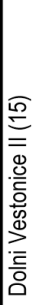 & 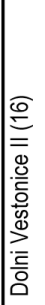 & & 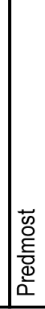 & 咅 & 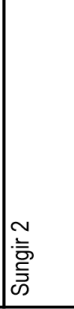 & 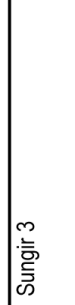 & 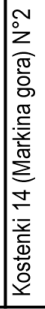 & 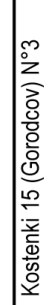 & 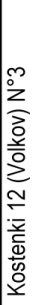 & & 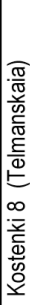 & 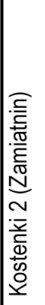 & 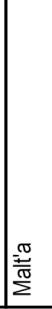 \\
\hline
\end{tabular}

Tableau 18 - Europe centrale et orientale : objets associés aux vestiges humains. ? : donnée inconnue ou incertaine. Tableau 18 - Central and eastern Europe: Associated objects with human remains. ? : data uncertain or unknown. 


\begin{tabular}{|l|r|r|r|r|r|r|r|r|r|r|r|r|r|}
\hline Age & Italie & $\begin{array}{c}\text { France, } \\
\text { Portugual, } \\
\text { Gr. Bret. }\end{array}$ & $\begin{array}{r}\text { Eur.W } \\
\text { (total) }\end{array}$ & $\begin{array}{c}\text { Coll. } \\
\text { Pavlov }\end{array}$ & Brno & Pred. & Aut. & Russie & Kostenki & $\begin{array}{c}\text { Eur. } \\
\text { Centr./Or. } \\
\text { (sans } \\
\text { Pred.) }\end{array}$ & $\begin{array}{c}\text { Eur. } \\
\text { Centr./Orient. } \\
\text { (total) }\end{array}$ & $\begin{array}{r}\text { Europe } \\
\text { (Total) }\end{array}$ & $\begin{array}{c}\text { Europe } \\
\text { sans } \\
\text { Pred }\end{array}$ \\
\hline Adultes & $70 \%$ & $68 \%$ & $69 \%$ & $83 \%$ & & $43 \%$ & $0 \%$ & $33 \%$ & $40 \%$ & $47 \%$ & $45 \%$ & $56 \%$ & $62 \%$ \\
\hline 0 & $0 \%$ & $16 \%$ & $8 \%$ & $0 \%$ & & $10 \%$ & $100 \%$ & $11 \%$ & $20 \%$ & $21 \%$ & $14 \%$ & $11 \%$ & $12 \%$ \\
\hline $1-4 \mathrm{a}$ & $0 \%$ & $11 \%$ & $5 \%$ & $0 \%$ & & $7 \%$ & $0 \%$ & $11 \%$ & $0 \%$ & $5 \%$ & $6 \%$ & $6 \%$ & $5 \%$ \\
\hline $5-9 \mathrm{a}$ & $0 \%$ & $5 \%$ & $3 \%$ & $0 \%$ & & $3 \%$ & $0 \%$ & $11 \%$ & $20 \%$ & $5 \%$ & $4 \%$ & $3 \%$ & $3 \%$ \\
\hline $\begin{array}{l}10-14 \\
\text { a }\end{array}$ & $15 \%$ & $0 \%$ & $0 \%$ & $0 \%$ & & $30 \%$ & $0 \%$ & $33 \%$ & $20 \%$ & $16 \%$ & $24 \%$ & $14 \%$ & $5 \%$ \\
\hline $\begin{array}{l}15-19 \\
\text { a }\end{array}$ & $15 \%$ & $0 \%$ & $15 \%$ & $17 \%$ & & $7 \%$ & $0 \%$ & $0 \%$ & $0 \%$ & $5 \%$ & $6 \%$ & $10 \%$ & $12 \%$ \\
\hline Total & $100 \%$ & $100 \%$ & $100 \%$ & $100 \%$ & & $100 \%$ & $0 \%$ & $100 \%$ & $100 \%$ & $100 \%$ & $100 \%$ & $100 \%$ & $100 \%$ \\
\hline $0-14 \mathrm{a}$ & $15 \%$ & $32 \%$ & $15 \%$ & $0 \%$ & & $50 \%$ & $100 \%$ & $67 \%$ & $60 \%$ & $47 \%$ & $49 \%$ & $34 \%$ & $26 \%$ \\
\hline
\end{tabular}

Tableau 19 - Europe, âge des inhumés.

Tableau 19 - Europe, distribution by Age at death.

\begin{tabular}{|l|r|r|r|r|r|r|r|r|r|r|r|r|r|}
\hline Sexe & Italie & $\begin{array}{c}\text { France, } \\
\text { Portugual, } \\
\text { Gr. Bret. }\end{array}$ & $\begin{array}{c}\text { Eur.W } \\
\text { (total) }\end{array}$ & $\begin{array}{c}\text { Coll. } \\
\text { Pavlov }\end{array}$ & Brno & Pred. & Aut. & Russie & Kostenki & $\begin{array}{c}\text { Eur. } \\
\text { Centr./Or. } \\
\text { (sans } \\
\text { Pred.) }\end{array}$ & $\begin{array}{c}\text { Eur. } \\
\text { Centr./Orient. } \\
\text { (total) }\end{array}$ & $\begin{array}{c}\text { Europe } \\
\text { (Total) }\end{array}$ & $\begin{array}{c}\text { Europe } \\
\text { sans } \\
\text { Pred }\end{array}$ \\
\hline Femmes & $25 \%$ & $16 \%$ & $21 \%$ & $17 \%$ & & $3 \%$ & & $0 \%$ & $0 \%$ & $5 \%$ & $4 \%$ & $11 \%$ & $16 \%$ \\
\hline Hommes & $15 \%$ & $21 \%$ & $18 \%$ & $67 \%$ & & $13 \%$ & & $8 \%$ & $0 \%$ & $26 \%$ & $18 \%$ & $18 \%$ & $21 \%$ \\
\hline Hommes? & $10 \%$ & $0 \%$ & $5 \%$ & $0 \%$ & & $0 \%$ & & $17 \%$ & $40 \%$ & $11 \%$ & $4 \%$ & $5 \%$ & $7 \%$ \\
\hline $\begin{array}{l}\text { IND. } \\
\text { (A+IM) }\end{array}$ & $50 \%$ & $63 \%$ & $56 \%$ & $17 \%$ & & $83 \%$ & & $50 \%$ & $60 \%$ & $58 \%$ & $73 \%$ & $66 \%$ & $57 \%$ \\
\hline Total & $100 \%$ & $100 \%$ & $100 \%$ & $100 \%$ & & & & $75 \%$ & $100 \%$ & $100 \%$ & $100 \%$ & $100 \%$ & $100 \%$ \\
\hline
\end{tabular}

Tableau 20 - Europe : Sexe des inhumés (IND : sexe indéterminé ; A. Adultes ; IM : Immatures).

Tableau 20 - Europe: Sex (IND : indeterminate ; A. Adults ; IM : Immatures).

\begin{tabular}{|l|r|r|r|r|r|r|r|r|r|r|r|}
\hline Age & Italie & $\begin{array}{l}\text { France, } \\
\text { Portug., } \\
\text { Gr. } \\
\text { Bret. }\end{array}$ & $\begin{array}{l}\text { Eur.W } \\
\text { (total) }\end{array}$ & $\begin{array}{l}\text { Coll. } \\
\text { Pavlov }\end{array}$ & Brno & Pred. & $\begin{array}{l}\text { Russie, } \\
\text { Autr. }\end{array}$ & $\begin{array}{l}\text { Eur. C- } \\
\text { Or.(sans } \\
\text { Pred.) }\end{array}$ & $\begin{array}{l}\text { Eur. } \\
\text { C./Or. } \\
\text { (total) }\end{array}$ & $\begin{array}{l}\text { Europe } \\
\text { (Total) }\end{array}$ & $\begin{array}{l}\text { Europe } \\
\text { sans } \\
\text { Pred }\end{array}$ \\
\hline Adultes & $70 \%$ & $68 \%$ & $69 \%$ & $83 \%$ & & $43 \%$ & $10 \%$ & $47 \%$ & $45 \%$ & $56 \%$ & $62 \%$ \\
\hline 0 & $0 \%$ & $16 \%$ & $8 \%$ & $0 \%$ & & $10 \%$ & $13 \%$ & $21 \%$ & $14 \%$ & $11 \%$ & $12 \%$ \\
\hline $1-4$ a & $0 \%$ & $11 \%$ & $5 \%$ & $0 \%$ & & $7 \%$ & $3 \%$ & $5 \%$ & $6 \%$ & $6 \%$ & $5 \%$ \\
\hline $5-9$ a & $0 \%$ & $5 \%$ & $3 \%$ & $0 \%$ & & $3 \%$ & $3 \%$ & $5 \%$ & $4 \%$ & $3 \%$ & $3 \%$ \\
\hline $\begin{array}{c}10-14 \\
\text { a }\end{array}$ & $0 \%$ & $0 \%$ & $0 \%$ & $0 \%$ & & $30 \%$ & $10 \%$ & $16 \%$ & $24 \%$ & $14 \%$ & $5 \%$ \\
\hline $\begin{array}{c}15-19 \\
\text { a }\end{array}$ & $30 \%$ & $0 \%$ & $15 \%$ & $17 \%$ & & $7 \%$ & $0 \%$ & $5 \%$ & $6 \%$ & $10 \%$ & $12 \%$ \\
\hline Total & $100 \%$ & $100 \%$ & $100 \%$ & $100 \%$ & & $100 \%$ & $40 \%$ & $100 \%$ & $100 \%$ & $100 \%$ & $100 \%$ \\
\hline $0-14$ a & $30 \%$ & $32 \%$ & $15 \%$ & $0 \%$ & & $50 \%$ & $30 \%$ & $47 \%$ & $49 \%$ & $34 \%$ & $26 \%$ \\
\hline
\end{tabular}

Tableau 21 - Europe : Age des inhumés (\% selon les classes d'âge, les régions et les sites).

Tableau 21 - Europe: Age at death (\% according to age at death class, region and site). 


\begin{tabular}{|l|r|r|r|r|r|r|r|r|r|r|r|}
\hline Sexe & Italie & $\begin{array}{l}\text { France, } \\
\text { Portug., } \\
\text { Gr. } \\
\text { Bret. }\end{array}$ & $\begin{array}{l}\text { Eur.W } \\
\text { (total) }\end{array}$ & $\begin{array}{l}\text { Coll. } \\
\text { Pavlov }\end{array}$ & Brno & Pred. & $\begin{array}{l}\text { Russie, } \\
\text { Autr. }\end{array}$ & $\begin{array}{l}\text { Eur. C- } \\
\text { Or.(sans } \\
\text { Pred.) }\end{array}$ & $\begin{array}{l}\text { Eur. } \\
\text { C./Or. } \\
\text { (total) }\end{array}$ & $\begin{array}{l}\text { Europe } \\
\text { (Total) }\end{array}$ & $\begin{array}{l}\text { Europe } \\
\text { sans } \\
\text { Pred }\end{array}$ \\
\hline Femmes & $25 \%$ & $16 \%$ & $21 \%$ & $17 \%$ & & $3 \%$ & $0 \%$ & $5 \%$ & $4 \%$ & $11 \%$ & $16 \%$ \\
\hline Hommes & $15 \%$ & $21 \%$ & $18 \%$ & $67 \%$ & & $13 \%$ & $8 \%$ & $26 \%$ & $18 \%$ & $18 \%$ & $21 \%$ \\
\hline Hommes? & $10 \%$ & $0 \%$ & $5 \%$ & $0 \%$ & & $0 \%$ & $17 \%$ & $11 \%$ & $4 \%$ & $5 \%$ & $7 \%$ \\
\hline IND.(A+IM) & $50 \%$ & $63 \%$ & $56 \%$ & $17 \%$ & & $83 \%$ & $75 \%$ & $58 \%$ & $73 \%$ & $66 \%$ & $57 \%$ \\
\hline Total & $100 \%$ & $100 \%$ & $100 \%$ & $100 \%$ & & $100 \%$ & $100 \%$ & $100 \%$ & $100 \%$ & $100 \%$ & $100 \%$ \\
\hline
\end{tabular}

Tableau 22 - Europe : Sexe des inhumés (\% selon les classes d'âge, les régions et les sites). (IND : sexe indéterminé ; A. Adultes ; IM : Immatures).

Tableau 22 - Europe: Sex (\% according to age at death class, region and site). (IND : indeterminate; A. Adults ; IM : Immatures). 